\title{
Dynamic Capacity Allocation for Airlines with Multi-Channel Distribution
}

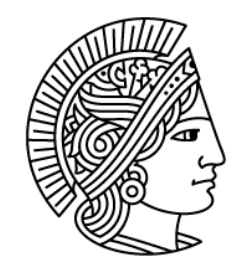

\author{
TECHNISCHE \\ UNIVERSITATT \\ DARMSTADT
}

\section{At the Department of Law and Economics of the Technischen Universität of Darmstadt}

submitted in fulfilment of the requirements for the degree of Doctor rerum naturalium

(Dr. rer. nat.)

Doctoral thesis

By Wang Weidi

First assessor: Prof. Dr. Dr. h.c. mult. Hans-Christian Pfohl

Second assessor: Prof. Dr. Jiazhen Huo

Third assessor: Jun.-Prof. Dr. Simon Emde 
Weidi Wang: Dynamic Capacity Allocation for Airlines with Multi-Channel Distribution

Darmstadt, Technischen Universität of Darmstadt

Year thesis published in TUprints 2019

Data of the viva voce: 19.11 .2018

Published under CC-BY-NC-SA 4.o International

https://creativecommons.org/licenses/ 
Contents

1 Introduction 7

1.1 Research background . . . . . . . . . . . . . . 7

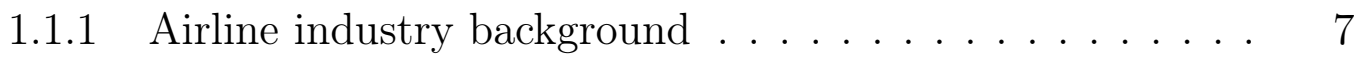

1.1.2 Airline distribution . . . . . . . . . . . . . . . . . 10

1.2 The concept of revenue management . . . . . . . . . . . 14

1.3 Customer shift behaviour . . . . . . . . . . . . . . . 15

1.4 Research objective and motivations . . . . . . . . . . . . 18

1.5 Research content and structure . . . . . . . . . . . . . . . 20

2 Literature review 23

2.1 Single-leg revenue management . . . . . . . . . . . . . . . . . 23

2.2 Revenue management with customer-shift behaviour . . . . . . 26

2.3 Network Revenue Management . . . . . . . . . . . . . . . . . . 27

2.4 Multi-Channel distribution . . . . . . . . . . . . . . . 30

2.5 Summary . . . . . . . . . . . . . . . . . . . 32

3 Dynamic capacity allocation for single-leg with Multi-Channel distribution 35

3.1 Single-leg capacity allocation model . . . . . . . . . . . . 35

3.1.1 Traditional Static Model For Single-leg Capacity allocation 35

3.1.2 Traditional dynamic Model for single-leg . . . . . . . . . 39

3.1.3 Control policy for single leg . . . . . . . . . . . . . 40

3.1 .4 Summary . . . . . . . . . . . . . . . . . . . . . 42

3.2 Dynamic capacity allocation for airlines with multi-channel distribution . . . . . . . . . . . . . . . . . . . 43

3.2 .1 Problem definition . . . . . . . . . . . . . . . . 43

3.2.2 Dynamic capacity allocation with a multi-channel model 44

3.2 .3 Optimal control policy for DCAM . . . . . . . . . . 44

3.3 Numerical experiment and analysis . . . . . . . . . . . . 47

3.3 .1 Design of experiments . . . . . . . . . . . . . 47

3.3.2 The effect of commission fee on airlines revenue . . . . . 48

3.3.3 The effect of channel amount . . . . . . . . . . . 50

3.3.4 Dynamic channel distribution with two fare classes and double channels . . . . . . . . . . . . . . . . 52

3.3.5 Channel allocation with different demand patterns . . . 54

3.4 Conclusion . . . . . . . . . . . . . . . . . . . 56 
4 DCAM Model with Channel-shift Customer Behaviour 59

4.1 Traditional capacity allocation model with customer behaviour . 59

4.1.1 Traditional Static buy-up model . . . . . . . . . . . . 59

4.1.2 Traditional dynamic buy-up model . . . . . . . . . . . 61

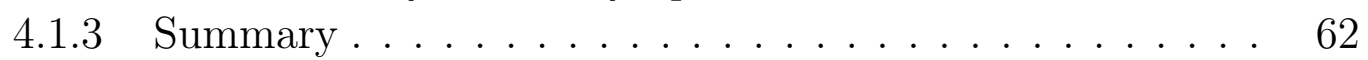

4.2 Problem definition . . . . . . . . . . . . . . . 63

4.3 Channel-shift Model . . . . . . . . . . . . . . . . . 64

4.4 Numerical experiments . . . . . . . . . . . . . . . . . 66

4.4.1 Design of experiments . . . . . . . . . . . . 66

4.4.2 Channel-shift in different shift rate . . . . . . . . . . 67

4.4.3 Channel-shift in different commission rate . . . . . . . 70

4.4.4 Channel-shift in different demand pattern . . . . . . . . 71

4.5 Conclusion . . . . . . . . . . . . . . . . . 75

5 Network capacity allocation with Multi-Channel distribution $\quad 77$

5.1 Traditional network model in revenue management . . . . . . . 77

5.1 .1 Dynamic network model . . . . . . . . . . . 78

5.1.2 Approximations based on dynamic network model . . . 79

5.1 .3 Summary..................... 80

5.2 Network capacity allocation with Multi-Channel distribution . . 81

5.2.1 Problem Definition and model . . . . . . . . . . . . 81

5.2 .2 Approximation method .............. 83

5.3 Asymptotic optimality of the NCDLP . . . . . . . . . . . . 84

5.4 Numerical examples . . . . . . . . . . . . . . . . . . . . 87

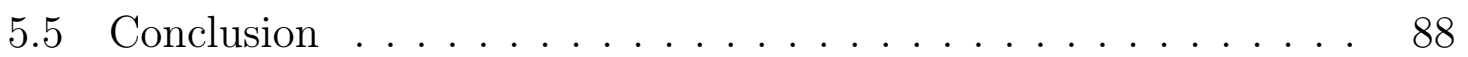

6 Conclusion and future study $\quad 91$

6.1 Conclusion ............................ 91

6.2 Future study . . . . . . . . . . . . . . . . . 94

7 Acknowledgement 95

$\begin{array}{ll}\text { Bibliography } & 95\end{array}$ 
List of Figures

1.1 China's aviation passenger traffic volume . . . . . . . . . 9

1.2 Top 10 increasing O-D markets in $2016 \ldots \ldots \ldots$. . . . . . 9

1.3 Customer ticket behaviour in airline industry . . . . . . . . . 10

1.4 Channel distribution system . . . . . . . . . . . . . . . 11

1.5 Airlines believe some distribution disruption will occur by 2021 . 12

1.6 Global channel share volume,2012-2017 . . . . . . . . . . . . 13

1.7 Global channel share volume,2017-2021 . . . . . . . . . . . . 13

1.8 Revenue management process flow . . . . . . . . . . . . . 16

1.9 Price comparison website . . . . . . . . . . . . . 17

1.10 Distribution business issues frustrate airline executives . . . . . 18

1.11 Structure of the Thesis . . . . . . . . . . . . . . . . 22

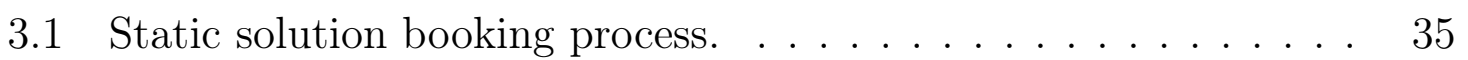

3.2 Dynamic solution booking process . . . . . . . . . . . . 36

3.3 Littlewood's model booking process . . . . . . . . . . . . 37

3.4 Fare class from Shanghai to Frankfurt . . . . . . . . . . . . 38

3.5 Partitioned and Nested booking limits and protection level . . . 41

3.6 Sales Behaviours in Experiment 3.3.2 . . . . . . . . . . . . 50

3.7 Revenue Changes over Different Commission Rates . . . . . . . 51

3.8 Sales Behaviour in Experiment 3.3.3 . . . . . . . . . . . . 52

3.9 Sales Behaviours Shown by Fares in Channels . . . . . . . . . . . 54

3.10 Sales Behaviours for Channels and Fares Separately . . . . . . . 55

3.11 Low Demand Pattern Sales Behaviours . . . . . . . . . . . . . 56

3.12 Middle Demand Pattern Sales Behaviours . . . . . . . . . . . 57

3.13 High Demand Pattern Sales Behaviours . . . . . . . . . . . 57

4.1 Static two-class buy up model booking process . . . . . . . . . 60

4.2 Channel Shift Decision Process . . . . . . . . . . . . . . . . 63

4.3 Low Shift Rate Sales behaviour for DCAMS . . . . . . . . . . . . 69

4.4 Middle Shift Rate Sales behaviour for DCAMS . . . . . . . . . . 69

4.5 High Shift Rate Sales behaviour for DCAMS . . . . . . . . . 70

4.6 Revenue comparison over different commission rate . . . . . . 71

4.7 Sales behaviour for low demand in DCAMS . . . . . . . . . 72

4.8 Sales behaviour for middle demand in DCAMS . . . . . . . . 73

4.9 Sales behaviour for high demand in DCAMS . . . . . . . . 74

5.1 A simple airline network . . . . . . . . . . . . . . . 77

5.2 Network for experiment . . . . . . . . . . . . . . . . . 87 
5.3 Revenue comparison in different commission rate . . . . . . . 89 
List of Tables

1.1 Civil aviation routes and number of aircraft statistics in China . 7

2.1 Single leg capacity allocation literature . . . . . . . . . . . . 24

2.2 Customer-shift behaviour literature . . . . . . . . . . . 27

2.3 Network capacity allocation literature. . . . . . . . . . . . . . 28

2.4 Multi-Channel literature . . . . . . . . . . . . . . . . . . . 31

3.1 Experiment list . . . . . . . . . . . . . . . . . . . 48

3.2 Channel Characteristics for Experiment $1 \ldots \ldots$. . . . . . . . . . 48

3.3 Results of Experiment $3.3 .2 \ldots \ldots \ldots \ldots$

3.4 Results of Experiment $3.3 .3 \ldots \ldots \ldots \ldots$. . . . . . . . . . . . . . . . . . . . . . . . . 51

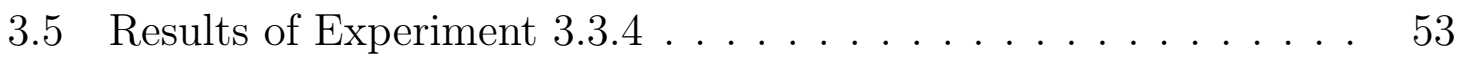

3.6 Results of Experiment $3.3 .5 \ldots \ldots \ldots \ldots$

4.1 Experiment list . . . . . . . . . . . . . . . . . . 67

4.2 Channel Characteristics for Experiment $4.4 .2 \ldots \ldots$. . . . . . 67

4.3 Results of Experiment 4.4.2 f . . . . . . . . . . . . . . . 68

4.4 Results of Experiment $4.4 .4 \ldots \ldots \ldots$. . . . . . . . . . . . . . . . . . . . . . .

5.1 Resource Characteristics for Experiment . . . . . . . . . . . . 87

5.2 Products Characteristics for Experiment . . . . . . . . . . . 88

5.3 Channel Characteristics for Experiment ........... 88 


\section{Denotation}

RM Revenue Management

DP Dynamic programming

DLP Determined Linear Programming

RLP Random Linear Programming

O-D Origin to Destination

GDS Global Distribution System

IATA International Air Transport Association

TMC Travel Management Company

OTA Online Travel Agency

IT Internet Technology

LCC Low-Cost Carriers

leg One flight from one city to another city

Network One O-D routes will go through one hub or several hubs.

EMSR Expected Marginal Seat Revenue

DCAP Dynamic Capacity Allocation Procedure Model

PNLP Probabilistic Nonlinear Programming Model

IIM Integrating Internet Market

DCAM Dynamic Capacity Allocation with Multi-Channel Distribution

DCAMS DCAM with Customer-shift Behaviour

DNM Dynamic Network Model 
Abstract

In 2017, China's online e-commerce sales has already reached 29.16 trillion Yuan. While gaining huge benefits, it also poses great challenges for each industry. One of the biggest challenge is the change of sales channels. Of course, there are also huge opportunities between them. Among them, it is a huge impact on the perishable products industry such as the airline industry and the hotel industry. Because of the perishable of the product, both hotels and airlines want to be able to sell the product for a limited period of time and gain considerable benefits. Therefore, at the beginning of the industry, airlines and hotels hoped to sell their products through more channels and attracted channels to sell products by paying their commission fee. With the rapid development of e-commerce, more and more online channels are replacing traditional offline channels. The change of channels has brought great challenges to airline management and costs. For example, although online channels absorb more customer demand, the commission costs of airlines have increased significantly. In addition to the cost pressures imposed on airlines, the increase in channels has brought conflicts between channels and between channels and airlines. Some of the channels' behaviour has caused great losses to the airlines. For example: change the condition of retreat fee, increase ticket or room price, maliciously reduce the price to compete with the airline and so on. These behaviours have affected the airline's reputation and have also brought losses to the airlines. In order to deal with the challenges of online agents, the airline has also taken some corresponding measures, such as the opening of online direct marketing websites, direct sales APP and so on. However, the effect has not been very good and it is difficult to compete with online agents who have customer volume.

At the same time, we also see that the airline industry and hotels are also facing great competitive pressure. For example, the high-speed rail increases the competitive of civil transport markets. High-speed trains generally have higher on-time rates than aircraft and also high-speed rail stations are generally more convenient for customers in the city.Therefore, for passengers, high-speed rail has advantages in short trips. In addition, the emergence of low-cost airlines has also intensified competition in the civil aviation industry such as China's Spring Airlines, Europe's Easyjet and Ryanair. Therefore, recently reducing channel distribution costs has been concerned for many airlines which are facing fierce competition in airline markets.

In a long period since the 1970s, capacity control has always played a pivotal role in defining airlines market strategy. However, when airlines select distribution channels and make capacity allocation decisions, they still separately make 
different decisions. Hence, when a customer purchases a ticket from a channel with an appropriate fare class, the channel might not be an optimal channel from the airlines' perspective. When the airline sells a ticket in a right channel, the ticket price is probably not a right fare. Therefore, how to establish a better channel and fare class capacity control model has become the key for airlines to increase revenue.

This thesis is a study based on the above issues. The main work includes the following aspects:

At first, we studied the single-leg capacity allocation problem that considers the channel factor. Although the network revenue management has a lot of academic research and has been applied in international routes,for many domestic routes airlines still basically use single-leg revenue management system.In addition, from the historical development of revenue management, the single-leg revenue management model is the basic model of all revenue management models. Therefore, it is important to first establish a single-leg revenue management model that considers the channel issues. In this study, we will integrate channel distribution into dynamic capacity control model. The model can make channel decisions in conjunction with inventory and this is similar to the procedure shown in pure capacity allocation. The study has proposed an optimal policy basing on bid price that incorporates commission fee, price, and capacity. The numerical experiment results illustrate that introducing the channel distribution into airline revenue system can significantly improve the revenues and efficiently reduce the channel distribution cost for airlines. The numerical experiments demonstrate that airline revenues will increase more than $3 \%$ in a simple integrated system with two channels compared to the independent model. This study also analyses the reasons for improvements in different situations (such as multi-channels have better improvements than a single-channel and the model has a better match of channels and fare classes) so that management insights are obtained for airlines.

Secondly, we analyse customer demand behaviours and we find that customers will experience demand transfer behaviours when facing channels. In the Internet age, due to more transparent information, the customer's transfer behaviour has been continuously expanded. For customers, the transfer of channels is more likely to occur than the transfer of fare classes because they do not need to pay for it. Therefore, it is necessary to establish a better revenue management model to consider the customer's channel transfer behaviour.In this part, we added customer channel transfer behaviour based on the original single-leg dynamic capacity allocation model that considers channel issues. We also developed the optimal policy for this model and made some numerical experiments. The numerical experiments demonstrate that the customer shift behaviour can influence the results of the model and subsequently the decisions 
of airlines. In the general numerical result, the new model can increase $1.23 \%$ than the above channel model. At the same time, through the analysis of the results, the airlines are provided with corresponding suggestions to face the customer's choice behaviour. For example, the airline needs to increase the customer's transfer rate through some methods, such as joining a price comparison network and increasing policy incentives.

Thirdly, on the base of single-leg model, we propose a new network dynamic model to integrate network revenue management and channel distribution. To take a network structure airline, the airlines can make more revenue benefits comparing the single-leg method. Although the network dynamic model can make more improvements, the exact optimization is impossible for practical purposes because of the curse of dimensionality. Therefore, we use determined linear programming method for approximating to dynamic model. The numerical experiments demonstrate that the airline revenues can increase more than $3 \%$ in a simple network when the commission rate is $15 \%$ compared to the traditional network model.

In addition to the above studies, the paper also summarizes the original literature on revenue management and channel issues and proposes future research directions. 
1 Introduction

Revenue management is an important branch of operation management, and it is also one of the difficulties and hotspots in the management of scientific research. Through the introduction, we can understand our research background, research motivation, research content, and the theoretical and practical significance of this study. At the same time we will give the structural framework of our study.

\subsection{Research background}

1.1.1 Airline industry background

The civil aviation industry was the first industry to introduce a revenue management system. After the United States liberalized regulation of the airline industry in the 1970s, revenue management systems formally entered the civil aviation industry. The application of revenue management technology also brings great profit growth to airlines, and also promotes the development of American aviation industry.

Table 1.1: Civil aviation routes and number of aircraft statistics in China Source:China Civil Aviation Statistics (CAST,2017)

\begin{tabular}{c|ccccccc}
\hline & 1990 & 2000 & 2010 & 2011 & 2012 & 2014 & 2017 \\
\hline Scheduled flight routes & 437 & 1165 & 1880 & 2290 & 2457 & 3142 & 4418 \\
International routes & 44 & 133 & 302 & 443 & 381 & 490 & 803 \\
No.of countries & 24 & 33 & 54 & 58 & 52 & 48 & 60 \\
Domestic routes & 385 & 1032 & 1578 & 1847 & 2076 & 2652 & 3615 \\
No.of cities & 94 & 139 & 175 & 178 & 180 & 198 & 229 \\
Scheduled flight Mileage $\left(\mathbf{1 0}^{4} \mathrm{Km}\right)$ & 50.7 & 150.3 & 276.5 & 349.1 & 328 & 463.72 & 1082.9 \\
International flights & 16.6 & 50.8 & 107 & 149.4 & 128.5 & 176.72 & 376.3 \\
Domestic flights & 32.9 & 99.5 & 169.5 & 199.6 & 199.5 & 287 & 706.6 \\
Passenger carried $\left(\mathbf{1 0}^{4}\right)$ & 1660 & 6722 & 26769 & 29500 & 31936 & 39195 & 55156 \\
No.of aircraft & 204 & 527 & 1597 & 1764 & 1941 & 2370 & 3296 \\
\hline
\end{tabular}

However, China's aviation market is relatively backward compared to the European and American aviation market. In the past, some regulatory measures 
on the policy also hindered the development of the civil aviation industry in China. With the new situation of China's policy opening up and China's rapid economic development, the development of the civil aviation industry has great potential. From the table 1.1, we can see that in the short 27 years from 1990 to 2017, the civil aviation industry has undergone tremendous changes in China. In 1990, the number of routes in China was only 437, and there were only 204 civilian aircraft. There were only 24 and 94 airline countries and cities.After nearly 28 years of development, by the end of 2017, China's air routes have increased to 4,418, which has increased by more than 10 times compared to 1990, and international air routes have also increased by 20 times to 490 . The number of civilian aircraft increased by a factor of 15 to 3,296 . It can be seen that China's civil aviation industry has developed very rapidly in these 20 years. Additionally, in the past 10 years, China's civil aviation industry has still developed rapidly. We can also look at the development of China's civil aviation industry in the past 10 years through the passenger traffic volume in the figure 1.1. In 2010, China's civil aviation passenger traffic was 290 million, and by 2017 it was close to 550 million passengers, an increase of nearly 2 times. In these 10 years, almost every year the volume of passenger traffic has maintained an increase of more than double digits. As can be seen from the 2016 IATA annual report(Tyler,2016)(see Figure 1.2), the domestic China passenger market saw the biggest incremental change in journey numbers, with 37 million more passenger journeys made in 2016 than in the year before. This increase was more than in the next two fastest-growing markets,domestic United States and domestic India combined.

However, behind the rapid development of civil aviation in China, the competition of airlines has increased due to the participation of private aviation and the development of high-speed railways. In 2013, with the launch of a number of new high-speed railways, China's high-speed rail business has reached 11,028 kilometres, and the 12,000-km high-speed railway is under construction. It has become the world's longest running high-speed railway and the country with the largest scale of construction.Due to short-term advantages, high-speed rail travel has become the first choice for many travellers, and therefore it poses great challenges for airlines.

The application of domestic airlines in related management techniques such as revenue management is not yet mature. In addition, due to the rise of the Internet, especially the development of the mobile Internet, all industries are facing new challenges and opportunities. Especially for the civil aviation industry, the Internet channels have completely changed the sales ecology of the civil aviation industry. Our research is aimed at such a situation. Integrating channel management into the revenue management system enables airlines to efficiently use existing resources to increase revenue. 


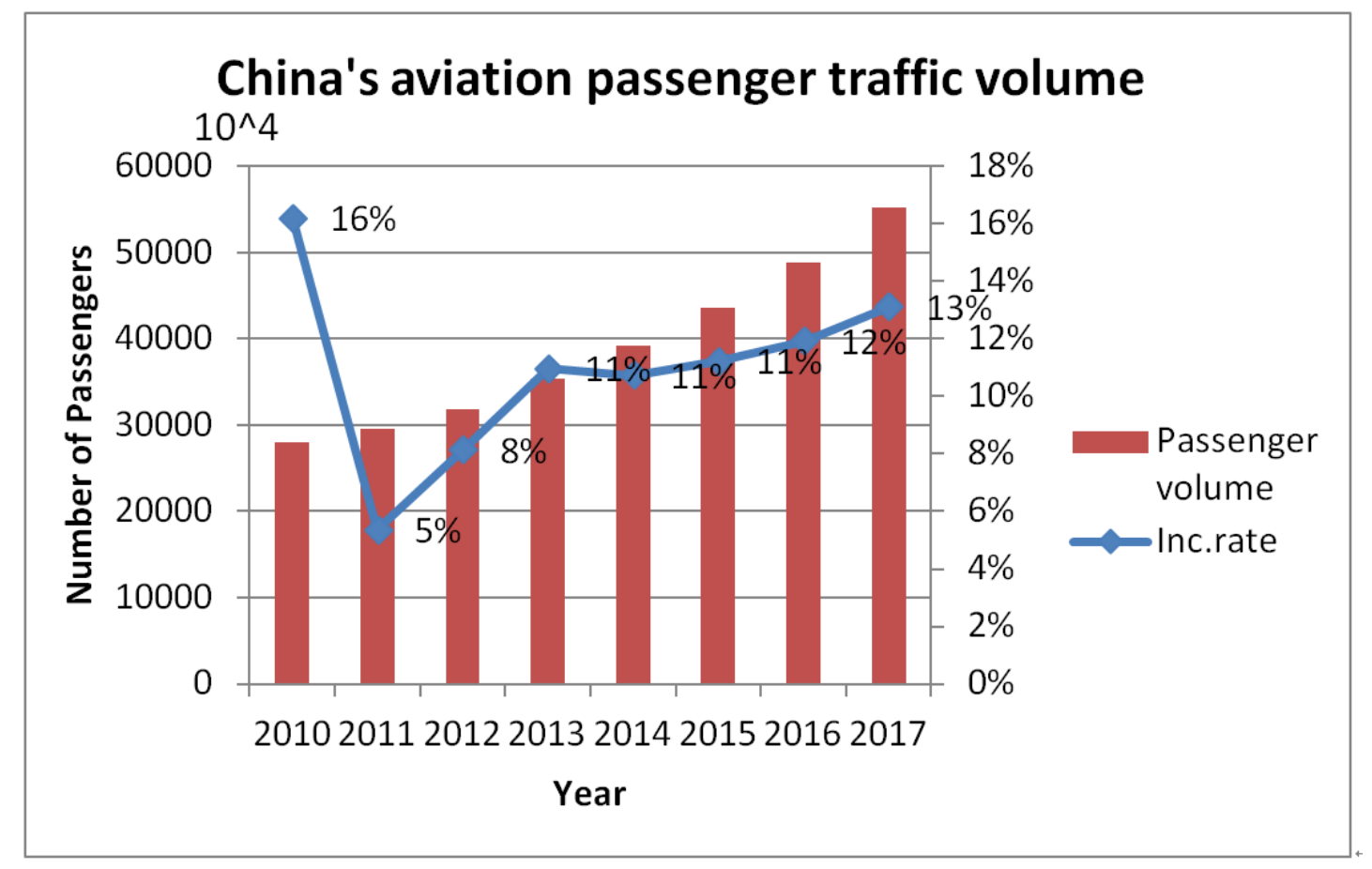

Figure 1.1: China's aviation passenger traffic volume Source:China Civil Aviation Statistics( CAST,2017)

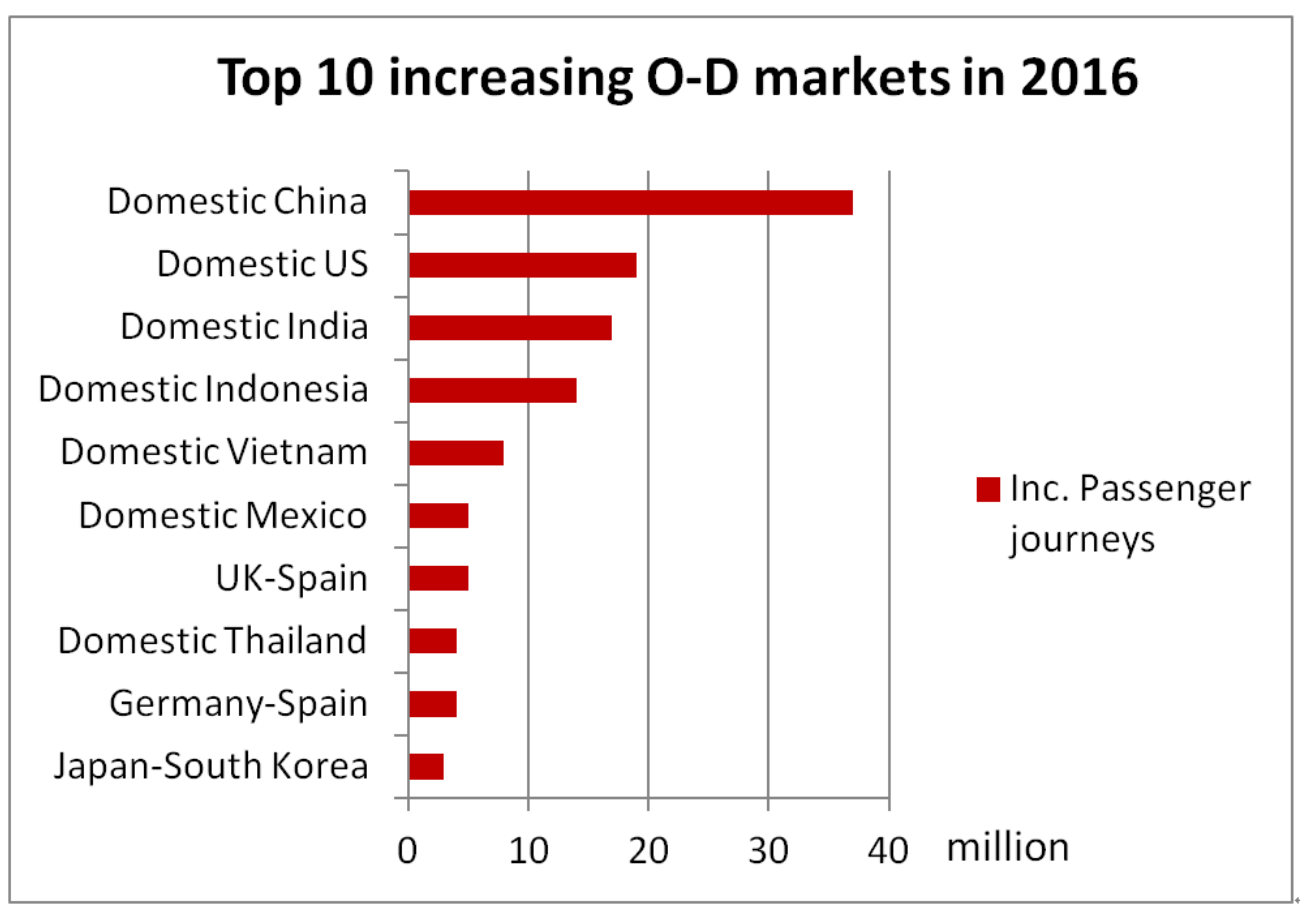

Source:IATA annual report(Tyler,2016)

Figure 1.2: Top 10 increasing O-D markets in 2016 


\subsubsection{Airline distribution}

With the development of e-commerce, many different sales channels have appeared in both traditional manufacturing and service industries. There are several channels for the traditional industry, such as offline channels Wal-Mart, Carrefour, online channel channels such as Taobao, JD and many companies have their direct sales channels, such as Apple, Topshop and so on.For the service industry, especially the aviation industry and the hotel industry, the emergence of e-commerce has greatly changed their sales channel model.Take the civil aviation industry as an example. Before 2000, consumers purchased airline tickets mainly through offline agents and call centres. It can be seen from the figure 1.3 that the call center has a very high volume growth in 2000. In 2000 , there was almost no increase in online channels. Since 2010, we have seen almost no increase in the growth of call centres and offline agents. In contrast, the growth of online channels has reached more than 50\%, and in 2015 it has even reached over $70 \%$. Therefore, we can see from the above data that the airline's channel has a great change with the development of e-commerce.In addition to the new demand for new online channels, the conflict and transparency brought about by the increase in channels have brought new challenges to the civil aviation industry.

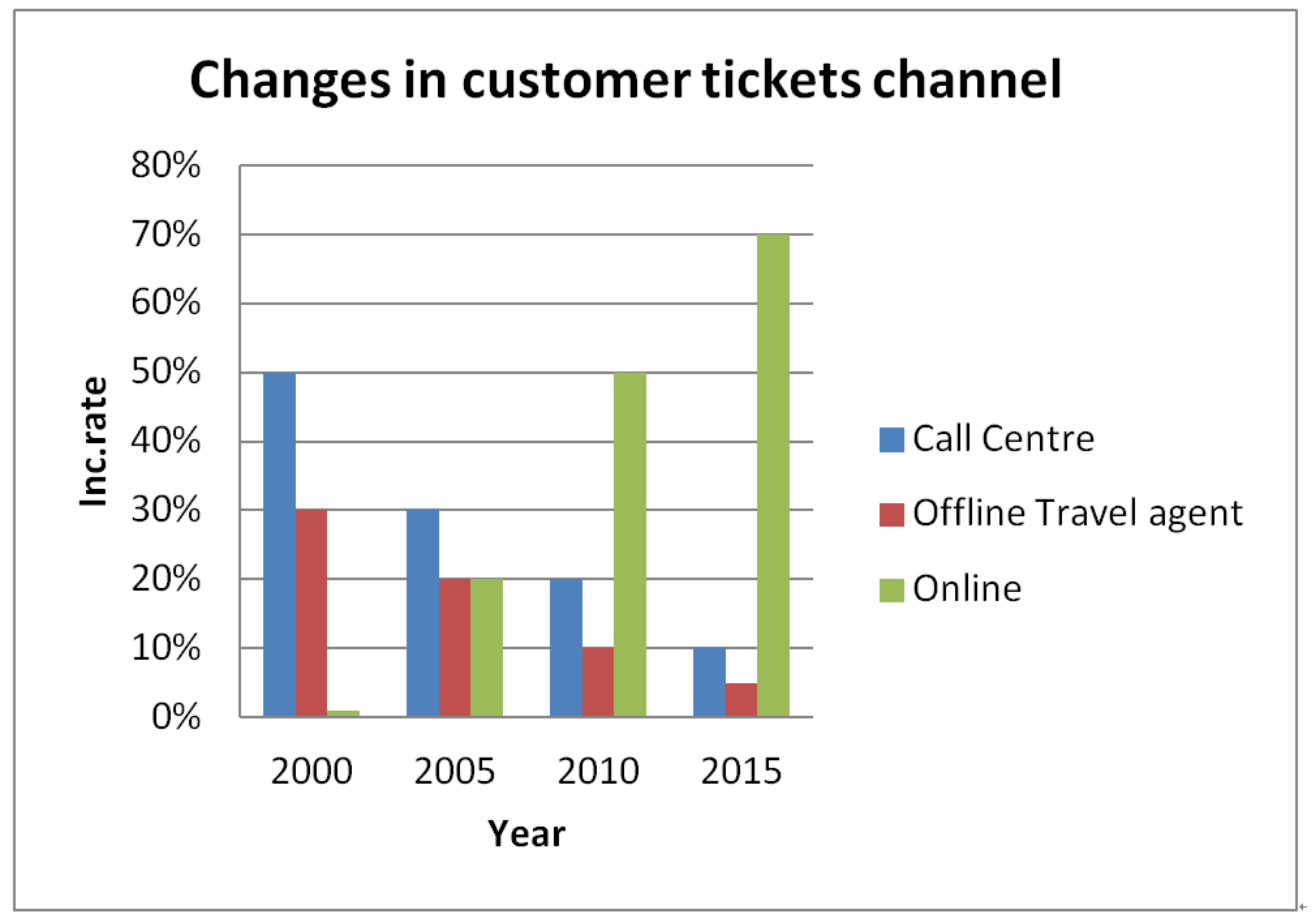

Source:IATA annual report(Tyler,2016)

Figure 1.3: Customer ticket behaviour in airline industry 
Firstly, we should introduce the channel structure of airline market, because the airlines sales channel is different compared with sales channels of traditional products. Airlines compete tremendously in increasing customer volume and maintaining market share in a limited time period. Hence, Airlines utilize many channels to reach customers in order to enhance the sales opportunities in a structure illustrated in Figure 1.4 (Harteveld,2012).

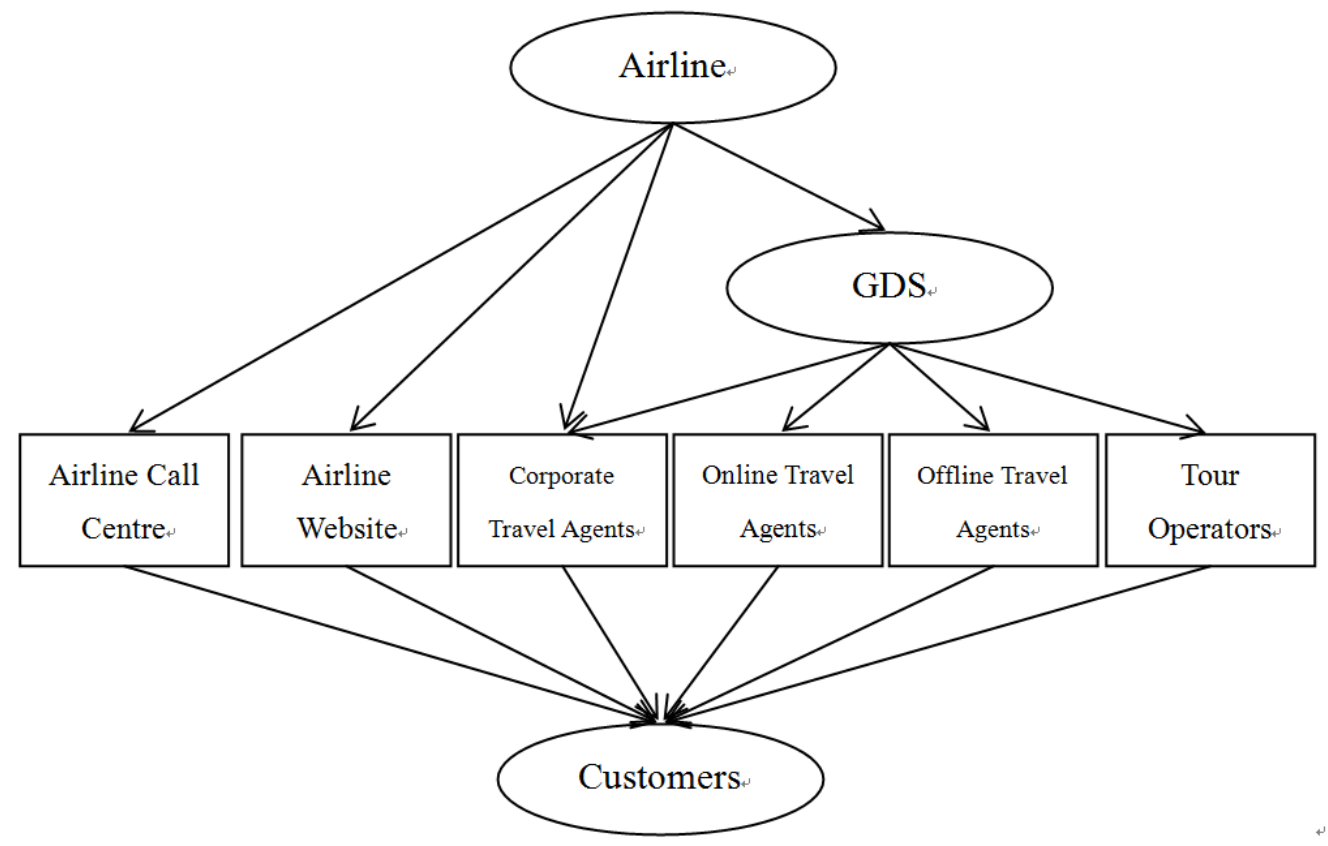

Figure 1.4: Channel distribution system

Normally, airlines sell their tickets through the Global Distribution Systems (GDSs), such as Sabre, Amadeus and Worldspan. A GDS is an intermediate company between travel service companies (such as airlines, hotels) and travel agents. GDSs collect information of airlines concerning supply inventory and price, then provide such information to travel agents and thereafter charge service fee to airlines. After retrieving information from GDSs, the indirect channel which includes off-line travel agents, online agents (such as Ctrip and Qunar in China, Priceline.com in USA and Opodo in Europe) and tour operators will receive tickets requests from customers. The commission fee of one ticket requested by travel agencies can vary from zero to $20 \%$ (including the GDS fee). In the highly competitive civil aviation sector, this has brought great costs to the airlines. Direct channels include airline websites, call centre and corporate travel clients. There is almost no cost for airlines. Therefore, some airlines have adopted strategies to increase the sales proportion of direct channel, such as improving the quality of website and increasing some ancillary service (Lufthansa,2005). In the IATA 2016 channel distribution report, 49 civil aviation executives were surveyed. One of the questions is asking 
what will happen to the distribution of channels(See figure 1.5). Increasing the direct sales channel got the highest score, followed by the application of the new revenue management system. From this perspective, it can also be shown that direct sales channels and revenue management are directly affecting civil aviation revenue in the opinion of civil aviation professional managers.

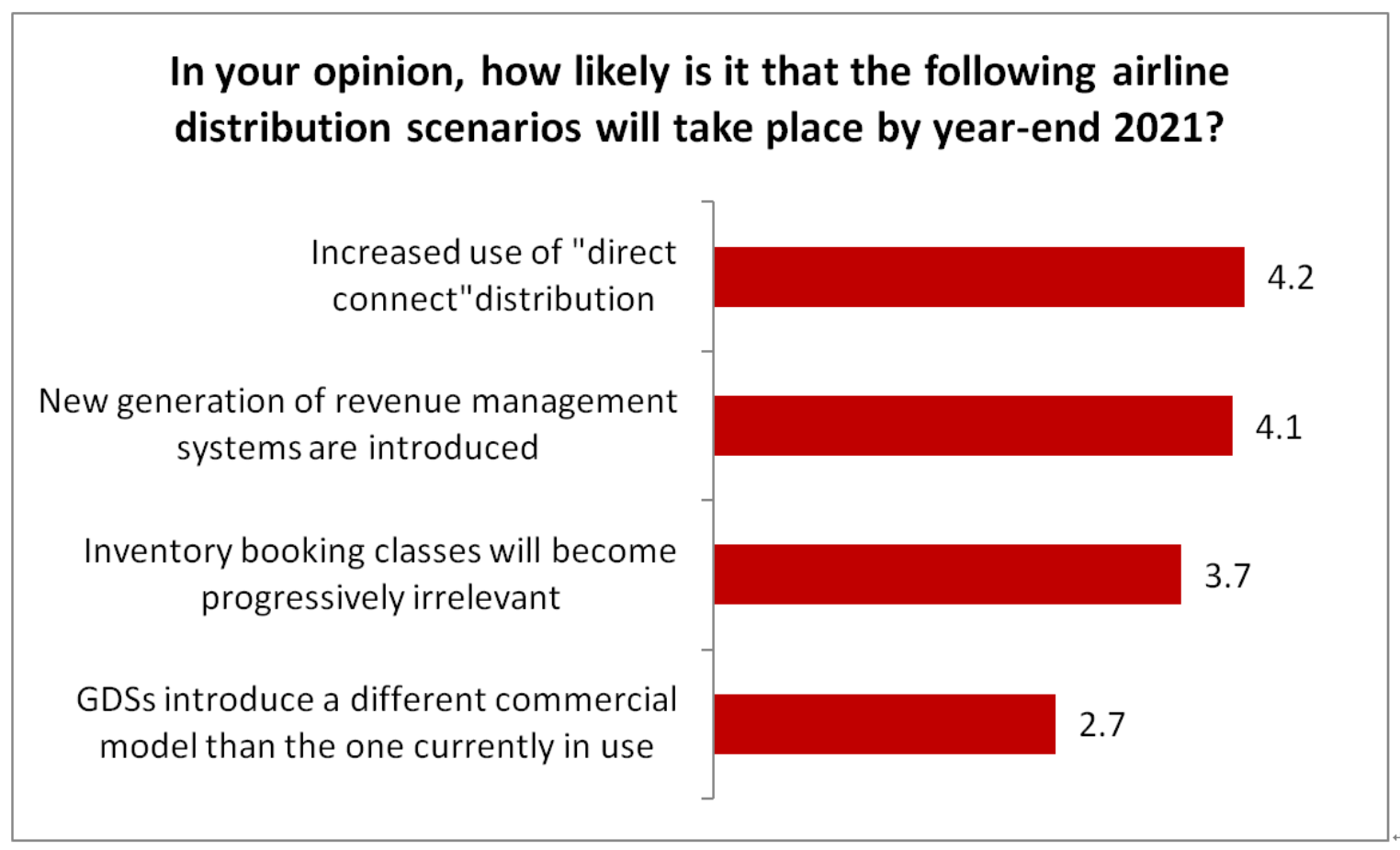

Source:The future of airline distribution,report by(Harteveld,2016)

Figure 1.5: Airlines believe some distribution disruption will occur by 2021

However, it is not easy to increase the use of direct sales channels. We can see that in the 2012 IATA report (See Figure 1.6), the direct sales channels at that time were around $40 \%$, while the indirect channels were above $55 \%$. The report also gives a forecast for the 2017 civil aviation channel based on the opinions of civil aviation executives. Civil aviation companies hope to increase direct sales channels to $55 \%$ and indirect channels to $45 \%$. However, in the 2016 report(See Figure 1.7), we found that the use of direct sales channels did not improve and remained basically the same as in 2012. Therefore, travel agents are still dominant in the channel distribution in airline industries.

There are three main reasons for this situation(Harteveld,2016). The business model of GDS could have been changed by airlines, but in the end it has not changed as a result of a profitable distributor. Second, airlines lack the will to transfer channel costs to travel agents. Because according to the traditional method of revenue management, aviation managers often ignore the cost and concern about the benefits. Finally, airlines often subcontract the channel dis- 


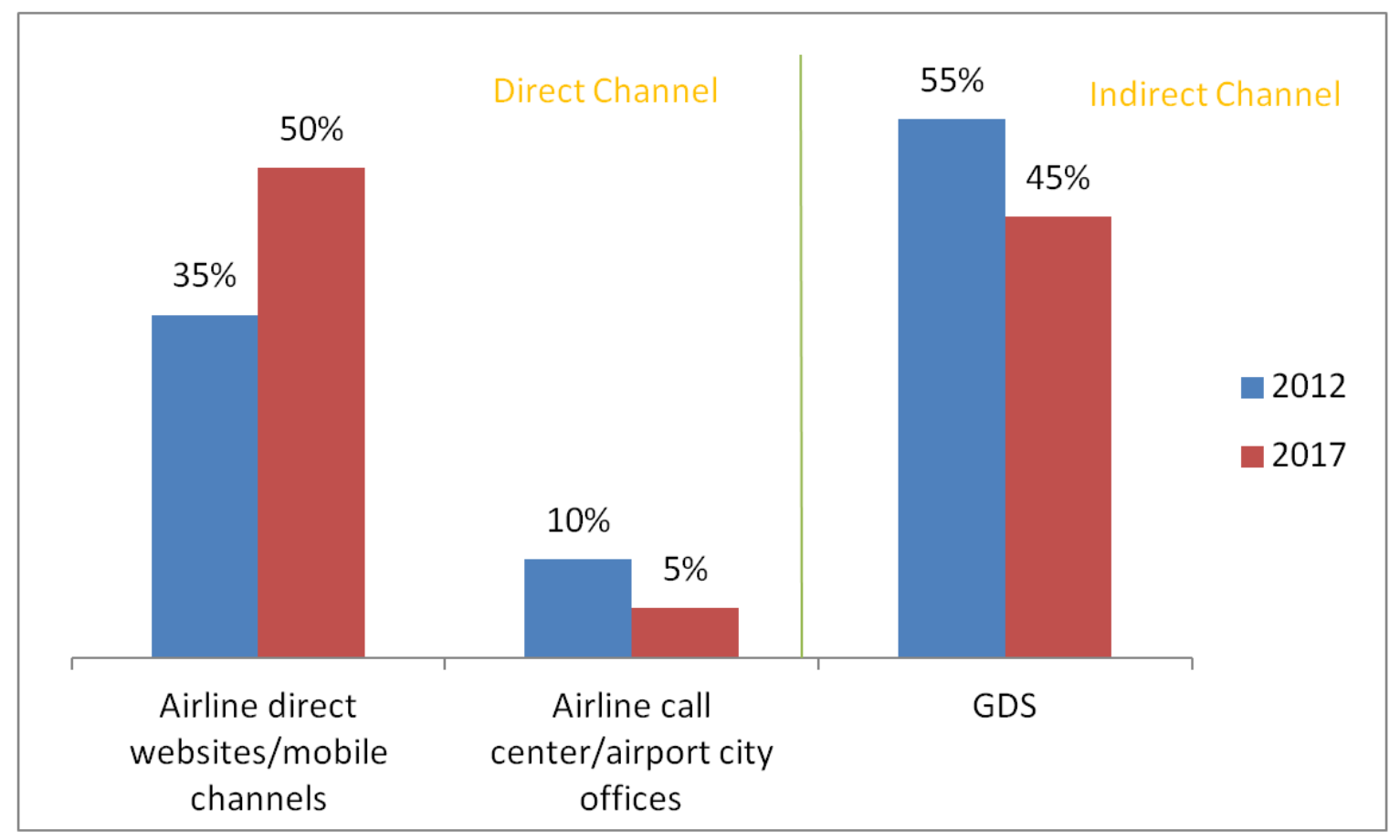

Source:The future of airline distribution,report by (Harteveld,2012)

Figure 1.6: Global channel share volume,2012-2017

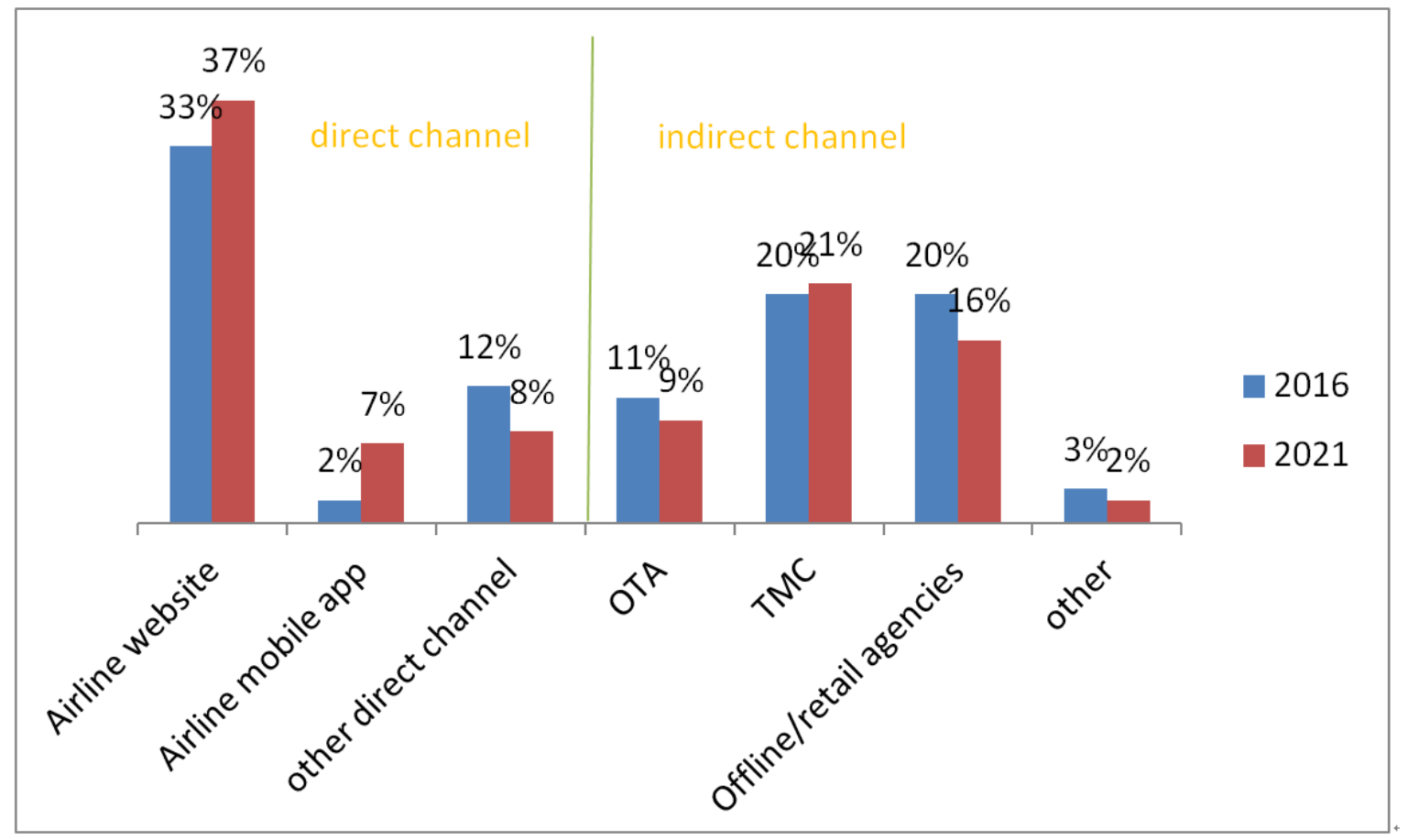

Source:The future of airline distribution,report by (Harteveld,2016)

Figure 1.7: Global channel share volume,2017-2021 
tribution system to GDS in order to reduce IT investment, making it difficult for airlines to control the channels. Under this current situation, airlines have to pay a large amount of commission fee for the indirect channel distribution every year. Therefore, as competition intensifies and channel conflicts intensify, how to adjust the channel structure to reduce costs and reduce conflicts has become a focus issue from the perspective of some airline companies.

\subsection{The concept of revenue management}

Revenue management has been widely practised for allocating perishable assets in service industries such as the airlines, car rentals and hotels since the deregulation of the airline industry in the 1970s. In the beginning, revenue management is used typically to determine how many seats should be reserved and offered for each class at different prices in one aircraft cabin. Since the application of this management technology, American airlines have acquired more profits in 1980s during which period almost all other airlines had a great deficit. Revenue management is a management technology that maximizes revenue, mainly by subdividing market demand, analysing consumer behaviours, establishing effective forecasting models, and determining the best selling prices and capacity for different consumers. There are four main application technologies for revenue management, which are also in the four directions of research: demand forecast, overbooking, dynamic pricing and capacity allocation.

Demand forecast:Demand forecasting is an important tool for revenue management. Accurate forecasting will provide data support for later overbooking management, pricing and inventory control. The main research methods include qualitative forecasting, time series, regression analysis and other statistical methods.

Overbooking: Overbooking means that more products are sold than actual products. As some customers may not arrive or cancel reservations during the sale of perishable goods such as air tickets, airlines will lose part of their profits. Therefore, airlines will adopt an overbooking strategy to offset this loss.

Dynamic pricing:Dynamic pricing refers to the strategy of the company to sell different products to different customers or different market segments at different prices according to market demand and its own supply capacity at different prices to maximize profit. This strategy distinguishes the previous static pricing strategies by meeting the market's needs by changing the differential prices at different times and in different markets.

Capacity allocation: Capacity allocation refers to the allocation of appropriate product quantities for different price levels. Since dynamic pricing usually takes into account the reaction of competitors, capacity allocation is more of a revenue management technology controlled by the enterprise itself. 
Although the four core technologies constitute revenue management, the core issue of revenue management is actually when and how much sold to whomever, which is the inventory control model. The research and development of the inventory control problem is mainly composed of two parts. First, it is a single resource issue, such as a single flight seat booking problem in airline industry, a hotel room booking problem in one night. The other is the multiple resource issue, which is what we call the network problem, such as the O\&D airline network problem or the hotel room reservation problem for a period of time.

In our research, a new capacity control model considering multiple channel factors was constructed based on the single resource and multiple resources issues, respectively.

Here we briefly introduce the application process of revenue management system. As shown in the figure 1.8, first of all, the airline will conduct data collection such as customer booking history, product data, and pricing data. The collected data is then input into the forecasting system to predict customer demand and behaviour. Then using the forecast data, the dynamic pricing system and the inventory control system are optimized to obtain price and inventory for each period. During this period, forecast data is used to decide whether to use the overbooking strategy and overbook the quantity. Then through the reservation system, different levels of price and inventory are released to each distribution system, such as GDS, airline website and call centre.

We can see that in the original revenue management process, the optimization part of the revenue management model did not consider the underlying channel issues at all. In fact, many channels will affect the airline's revenue and even make the revenue management system useless. Therefore, the research in this paper puts the channel problem into the revenue management system and optimizes it in a unified way to avoid the conflict between the channel and the revenue system.

This section is a brief introduction to revenue management. Specific related content can refer to the relevant book "Pricing and revenue optimization" (Phillips,2005) and "The theory and practice of revenue management" (Talluri and Van Ryzin,2006).

\subsection{Customer shift behaviour}

Similarly, based on the development of the Internet, customers can obtain more product information through more channels. Especially with the emergence of some comparison websites like Skyscanner in Europe, Priceline in American and Qunar in China, customers can obtain almost all ticket information from every channel through a website. As figure 1.9, if customer want to buy a flight ticket from Shanghai to Frankfurt, the customer can obtain the ticket information 


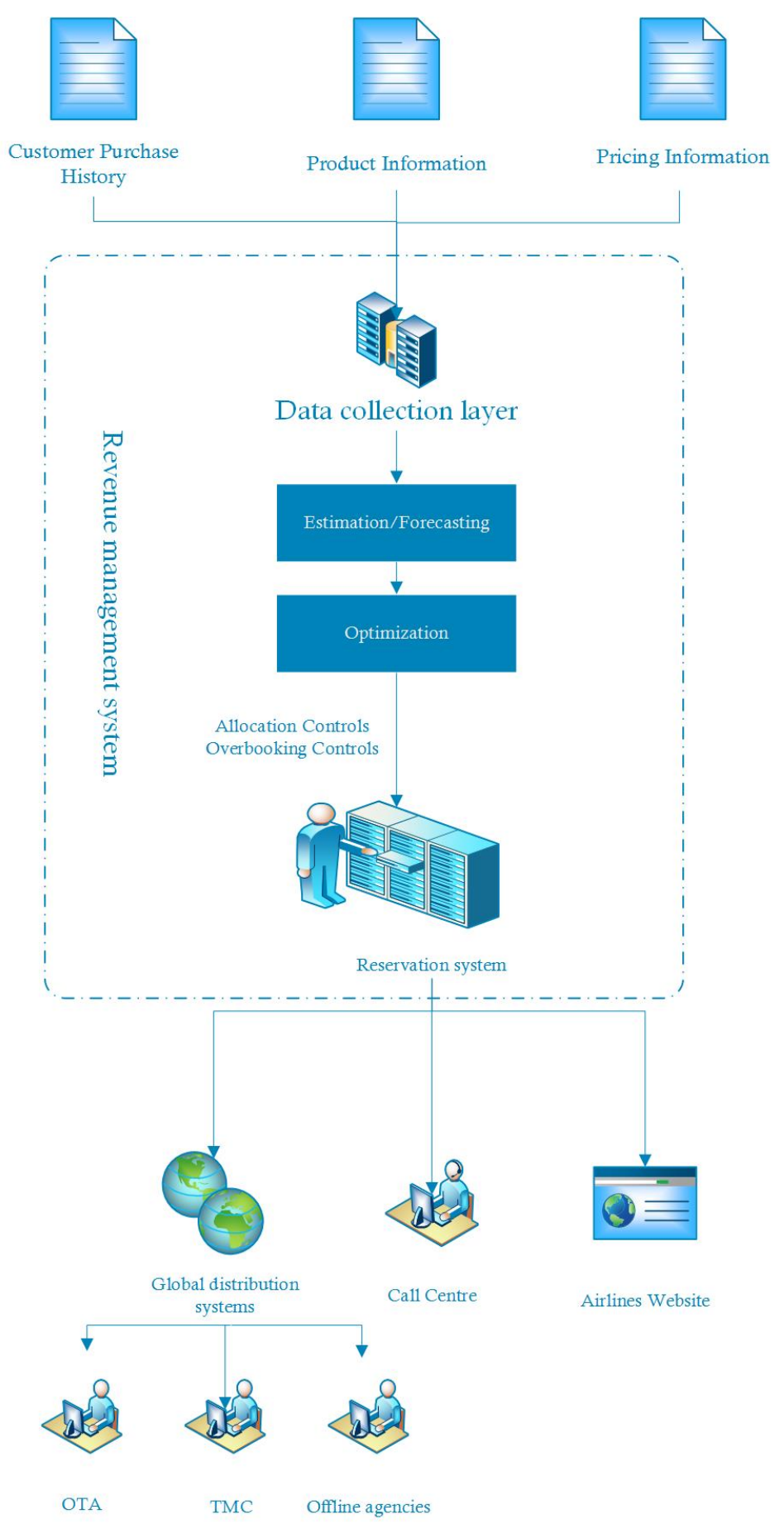

Source:(Talluri and Van Ryzin,2006)

Figure 1.8: Revenue management process flow 
and channel information from the Skyscanner. Therefore, the airline's product information is becoming more transparent.

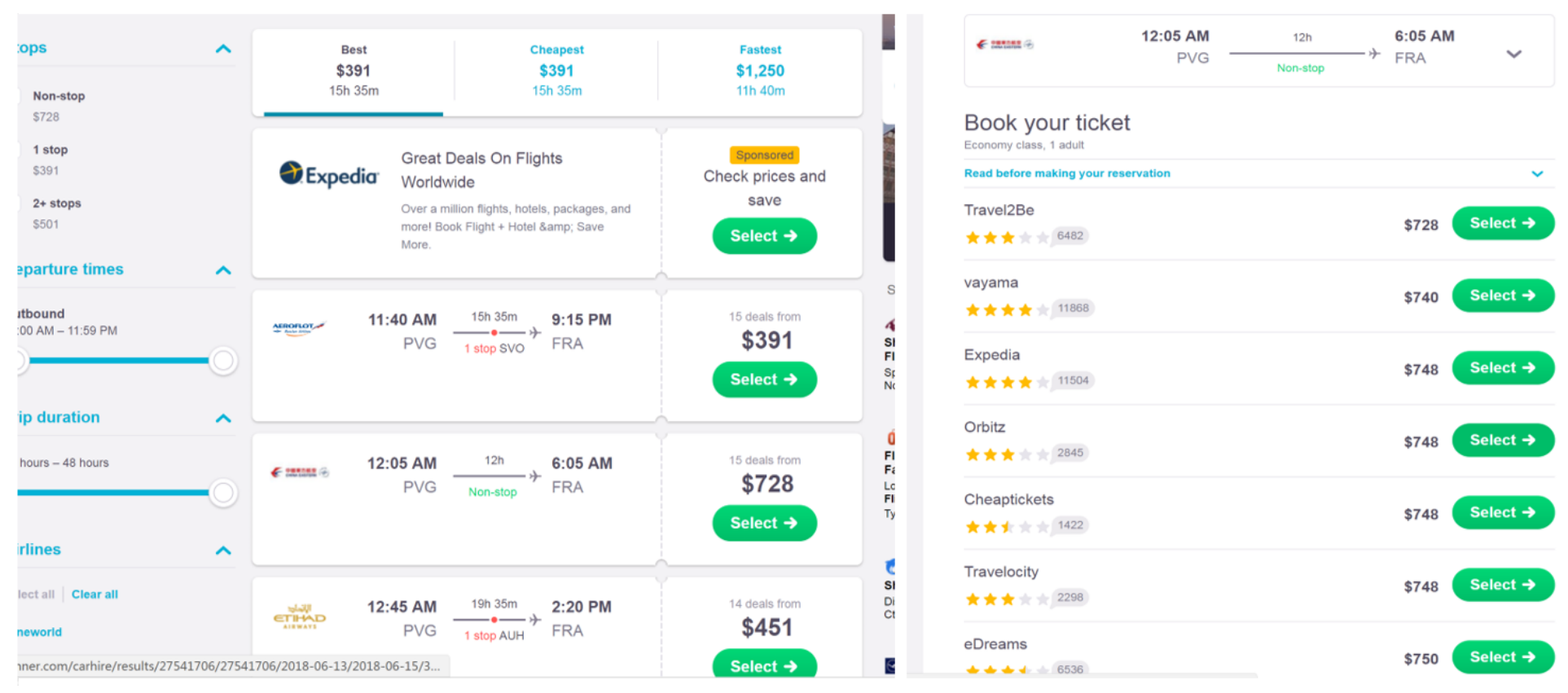

Figure 1.9: Price comparison website

Source: Skyscanner Website

And precisely because of the increase of information transparency, the customer's choice behaviour has a greater impact on airlines and channel manufacturers. Revenue management is the most important technology to deal with this challenge. Therefore, this concept of customer choice behaviour is proposed. Specifically, the customer's choice behaviour is that when the customer purchases an airline ticket, the demand shifts due to the impact of the information. Many scholars have incorporated customer selection behaviours into revenue management models. The most important of these is the two streams. The first is the customer's buy-up behaviour. When the customer is rejected or the low-level customer chooses to purchase upwards under certain conditions. The other is the choice model. The airline no longer provides a single product for different customers but provides a set of products for their selection. For the channel issues considered in this paper, the customer's choice behaviour has a greater impact on airline's revenue. When customers choose high-grade fares, their costs will increase, so there may not be many customers who choose to buy upwards. When customers choose low-cost channels such as airline direct sales channels, their cost will not increase and the transfer between channels is more likely to happen.So we consider the customer's choice behaviour in Chapter 4 in our model. More specific, Strauss et al. review the related theory and model in his paper(Strauss et al.,2018). 


\subsection{Research objective and motivations}

This research addresses the capacity allocation problem in different distribution channels with the airline industry as the background. In existing systems and models (see Figure 1.8), airlines use their revenue management system to select the capacity and price in different fare classes, and then the decisions of capacity and price are provided through indirect channels (Global Distribution Systems(GDSs) and travel agents) and direct channels (Airline websites). After that, customers can purchase tickets from travel agents or airline websites. In such a system, airlines use their revenue management system to optimize the inventory without considering the effects of distribution channels. Demand from different channels is always fulfilled, if possible, with the first come, first serve rule. At the same time, the travel agencies, without informing the airline company, always execute their capacity strategy with the aim of increasing their own profits. Some travel agencies even increase the product's price or decrease some fare capacities. This decentralized marketing strategy associated with travel agencies can sometimes damage the airline's revenue management system, and as a consequence, it even reduces the airline's revenues and increases the costs.

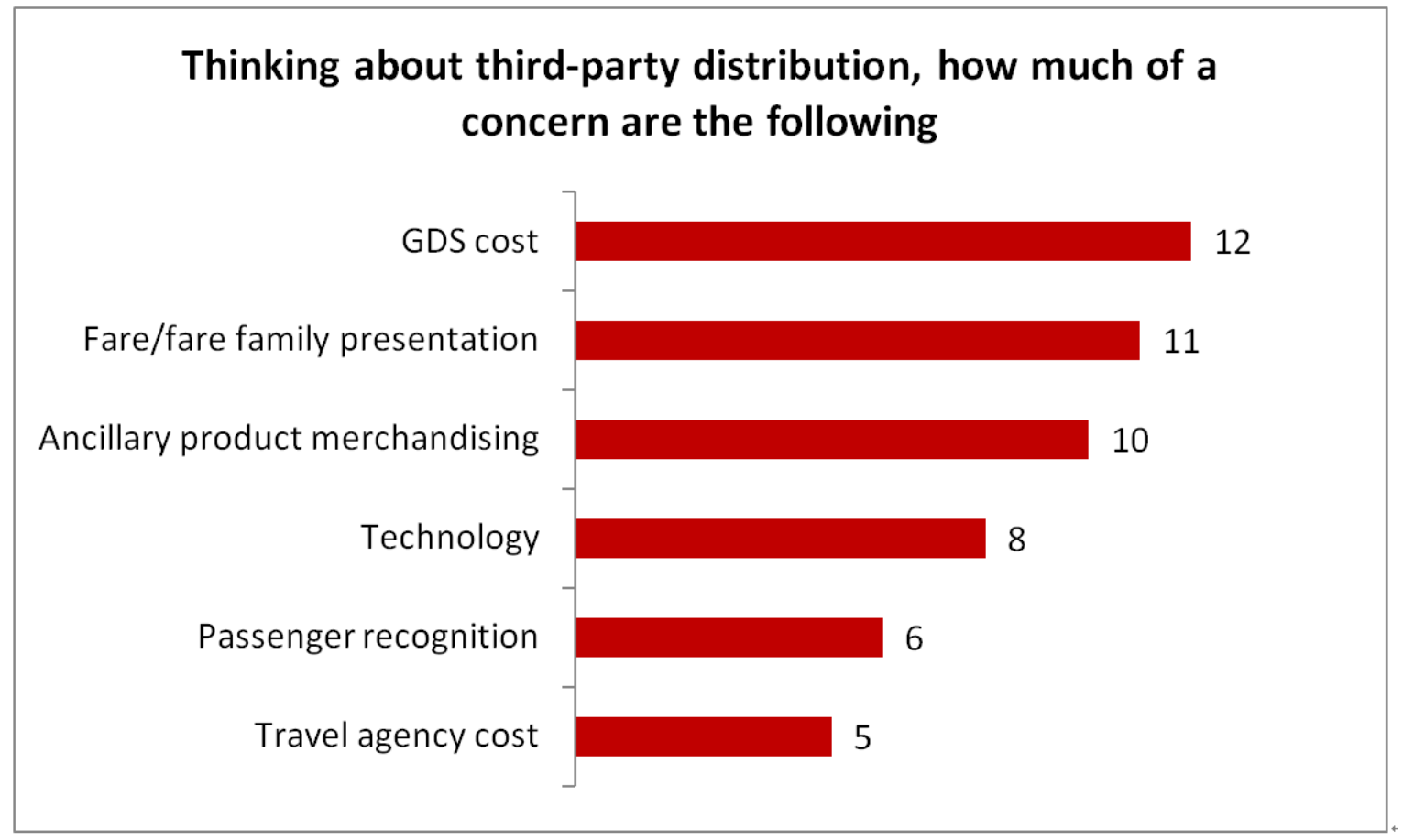

Source:The future of airline distribution,report by (Harteveld,2012)

Figure 1.10: Distribution business issues frustrate airline executives

One survey of the distribution channel issues with airline executives demonstrates that apart from the costs of indirect channels including GDS's fees 
and the commission fees of travel agencies, airline executives are also frustrated by the inadequate policy transparency of travel agencies that may often conflict with the aim of the revenue management policies of the airlines (Harteveld,2012). Thus, some airlines cut off all channels for lowering the costs and avoiding the conflict. For instance, some low-cost carriers (LCCs) only sell on their own websites. However, one immediate disadvantage is the loss of customers due to lack of indirect channels. Thus, some LCCs have tried to return to the traditional airline sales system. The example of LCCs implies that eliminating all indirect channels is not the best strategy to confront the market environment. It is significant for the airlines to establish an efficient capacity allocation model that integrates the revenue management system and the channel distribution system so that the decisions and optimization have a wider reach in order to cover interrelated issues in revenue management and channel distribution simultaneously. With the support of such a system, the airlines can allocate cabin capacity in different fare classes and different channels based on selling seasons and demand.

Therefore, the key question is

"How to integrate the channel distribution into revenue management system to reduce the cost and avoid conflicts with channels?"

More specifically, three research questions were addressed:

RQ1: how to integrate the channel factors into single-leg capacity allocation model?

-What is the current situation of single-leg capacity allocation model? -What is the new integration model? -What are the properties of the new model?

RQ2: how does the customer behaviour effect on the new model and how to solve it?

-What is the buy-up customer behaviour? -How to definite the buy-up behaviour in the new model?

RQ3: how to integrate the channel factors into network capacity allocation model?

-What is situation of the network capacity allocation? -What is the network integration model? -what are the properties of the network model?

There are plenty of literature on airlines' capacity allocation and channel distribution. But seldom research focus on the integration of channel factor and capacity allocation. However, in the reality, airlines have a deep effect on their revenues because of channel factor. Therefore this study proposes three new solutions to the original three basic revenue management problems, namely the single-leg problem, the single-leg problem taking into account the customer's buy-up behaviour, and network problem. 


\subsection{Research content and structure}

The main research content and distribution of this thesis are as follows:

In Chapter 1, the research mainly analyse the background of the development of the civil aviation industry and the current status of the channels. This is also the source of research and motivation for this study. At the same time, a brief introduction to the concept of revenue management is introduced. After that, the research introduce the customer choice behaviour. Finally, the content of the research and the structure of the paper are introduced.

In Chapter 2, we review four parts of academic research, namely single-leg revenue management, network revenue management, revenue management models for customer choice behaviour, and channel allocation related literature. Through summarizing and analysing the existing academic research, this paper proposes the contribution of this study to the academic field.

In Chapter 3, we propose a dynamic model to describe the decision behaviours when revenue management and channel distribution management in single-leg are integrated from the airlines' perspective. To enhance revenue management, we introduce factors to describe the channel distribution characteristics into a dynamic programming model. The model proposes a two-decision mechanism in the presence of the demand for one seat. One decision chooses the fare class, and the other selects the channel. With this fundamental mechanism in the model, we use the bid-price optimal policy to ensure that the airline maximizes their revenues. The new model can make airlines allocate the capacity efficiently among both different fare classes and different channels based on the demand and sales period.

This Chapter makes three contributions in the literature. First, we propose a dynamic capacity control model that integrates the revenue management and channel distribution decisions. The modelling framework can be further used for other relevant issues in the airlines. Second, we develop the optimal policy for the airlines on the basis of this model. The results can be implemented in practice. The numerical experiments demonstrate that airline revenues will increase more than $3 \%$ in a simple integrated system with two channels compared to the independent model. Further improvement is possible in more complicated situations. This study also analyses the reasons for improvements in different situations (such as multi-channels have better improvements than a single-channel and the model has a better match of channels and fare classes) so that management insights are obtained for airlines.

In Chapter 4,we further extend the study to consider the customer shift behaviours after being rejected by high-cost channels. The goal is to show how the customer shift behaviours influence our two-decision mechanism. Those models and mechanisms well reflect the airlines' decision behaviour and customer 
behaviour with the consideration of the channel distribution effect. Therefore, the results of this study can enhance the decision-making process of the airlines for the capacity allocation problem.

The numerical experiments demonstrate that the customer shift behaviour can influence the results of the model and subsequently the decisions of airlines. At the same time, through the analysis of the results, the airlines are provided with corresponding suggestions to better face the customer's choice behaviour. For example, how can an airline change when faced with different needs, and the purchase of a model by a customer can increase the airline's acceptance rate.

In Chapter 5,on the base of single-leg model, we propose a new network dynamic model to integrate network revenue management and channel distribution. To take a network structure airline, the airlines can make more revenue benefits comparing the single-leg method. This has been demonstrated by several researchers such asBelobaba and Lee(2000p),Belobaba(2001r), Williamson(1988c) and Williamson(1992a). Although the network dynamic model can make more improvements, the exact optimization is impossible for practical purposes because of the curse of dimensionality. Therefore, we use determined linear programming method for approximating to dynamic model.

This Chapter also makes three contributions in the literature. First, we propose a new network dynamic model which considered channel factor. Second, we develop an approximation method for the airlines. These methods can be directly used in airline industries. Third, the numerical experiments demonstrate that the airline revenues can increase more than $3 \%$ in a simple network when the commission rate is $15 \%$ compared to the traditional network model.

In Chapter 6, the research results of this study are summarized and the contribution of this thesis in the academic field and application field is elaborated. Finally, it points out the research insufficiency and further research direction. 


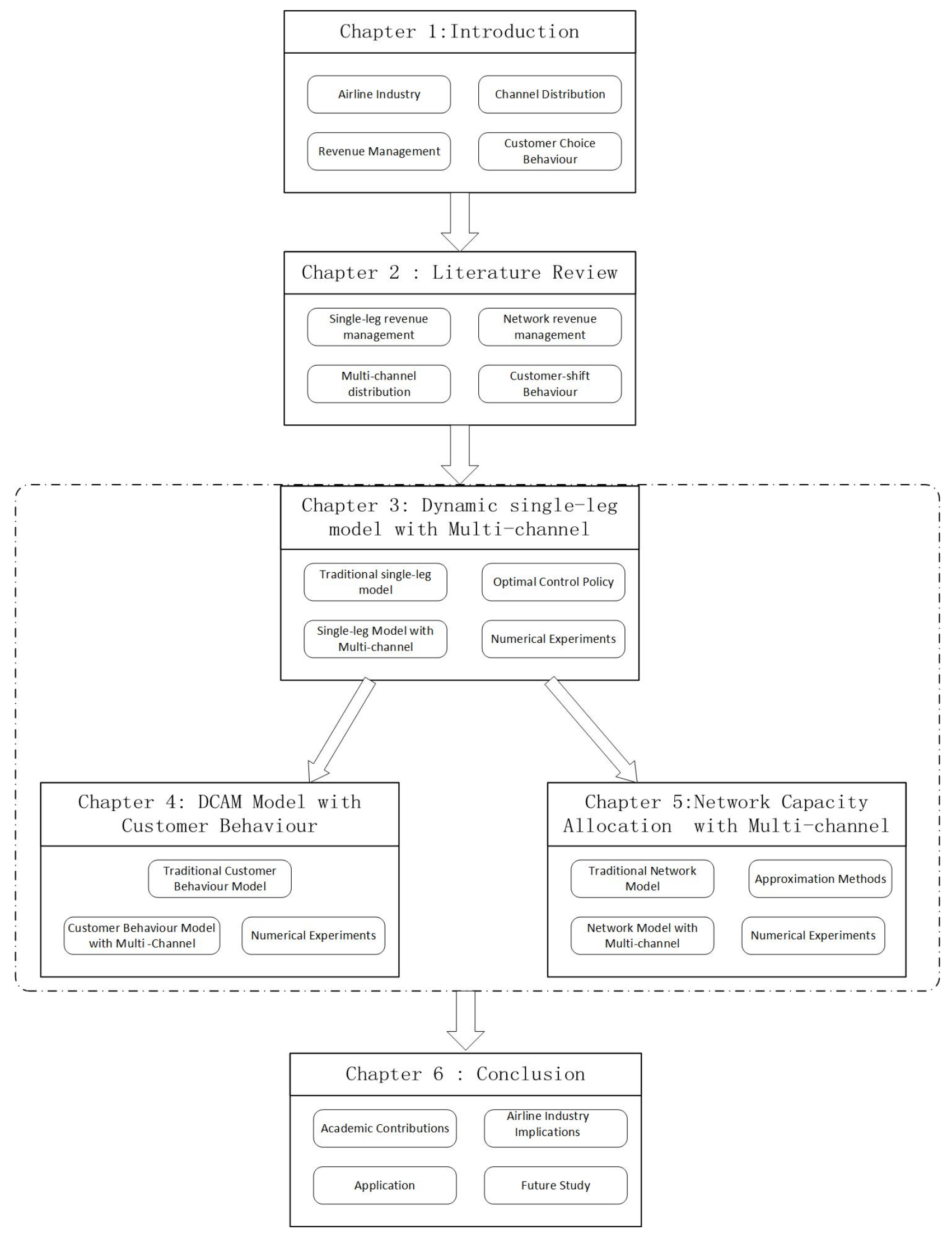

Figure 1.11: Structure of the Thesis 


\section{Literature review}

Through the introduction of the development of the civil aviation industry and the problems existing in the civil aviation channel in Chapter 1, we have initially understood the significance of integrating revenue management issues with channels. In this chapter we plan to determine the theoretical theory of this study by reviewing relevant academic research. We separately reviewed the literature on four relevant aspects, namely single-leg capacity control, network capacity control, capacity control considering consumer behaviour, and related research on channel distribution.

\subsection{Single-leg revenue management}

As mentioned before, capacity control as a traditional and significant aspect of revenue management has been reported in many studies. The earliest research on capacity control was Littlewood(1972) which proposed a static model for two fare classes on a single flight leg. Since then, many scholars have conducted research on the issue of single flight leg and have mainly divided into several issues (See Table 2.1). The first is the single-leg dual-class problem, which is what Littlewood(1972) has proposed. Bahatia et al.(1973) and Richter(1982) used different methods to derive the Littlwood rule. Mayer(1976) and Titze and Griesshaber(1983) separately conducted a simulation study of performance of Littlewood's rule. Mayer implied that if the Littlewood rule was reused before the flight took off, its effect might be the same as that of a more complex DP model. Titze and Griesshaber proved that the Littlewood rule is robust under the assumption of low to high fare.

Based on the two fare class single-leg model, Belobaba (1987a,b,1989) extended the two fare classes model to multiple fare classes model and developed an effective heuristics solution (EMSR, expected marginal seat revenue) to solve this single-leg problem. McGill(1989) and Wollmer proved that the approximation result is roughly good in some typical demand distribution based on the EMSR method. However, for general demand distribution, Robinson(1995) gave a evidence that the result is poor.After that, Van and McGill improved the EMSR method and proposed a close method EMSRb. The approximation result of EMSRb was closer to the result of optimal booking limits. EMSRb is then widely used in airline industries although they did not give an optimal booking limits. For obtaining optimal booking limits, some literatures provided different methods. Curry(1990) obtained the optimal booking limits in continuous demand distribution and Wollmer(1992) obtained the optimal 
Table 2.1: Single leg capacity allocation literature

\begin{tabular}{|c|c|c|}
\hline Category & Year & Author \\
\hline \multirow{5}{*}{ Static single leg two-class model } & 1972 & LITTLEWOOD \\
\hline & 1973 & Bhatia and Parekh \\
\hline & 1976 & Mayer \\
\hline & 1982 & Richter \\
\hline & 1983 & Titze and Griesshaber \\
\hline \multirow{9}{*}{ Static single leg Multi-class model } & $1987 \mathrm{a}$ & Belobaba \\
\hline & $1987 \mathrm{~b}$ & Belobaba \\
\hline & 1989 & Belobaba \\
\hline & 1989 & McGill \\
\hline & 1990 & Curry \\
\hline & 1993 & Brumelle and McGill \\
\hline & 1992 & Wollmer \\
\hline & 1995 & Robinson \\
\hline & 1998 & Van and McGillVan Ryzin and McGill \\
\hline \multirow{19}{*}{ Dynamic single leg model } & 1993 & Lee and Hersh \\
\hline & 1994 & Shaykevich \\
\hline & 1994 & Young and Van Slyke \\
\hline & 1997 & Brumelle and Walczak \\
\hline & 1998 & Kleywegt and Papastavrou \\
\hline & 1999 & Zhao \\
\hline & 1999 & Subramanian et al. \\
\hline & 1999 & Liang \\
\hline & 1999 & Lautenbacher and Stidham Jr \\
\hline & 1999 & Zhao \\
\hline & 2000 & Van Slyke and Young \\
\hline & 2001 & Kleywegt and Papastavrou \\
\hline & 2003 & Brumelle and Walczak \\
\hline & 2009 & Gallego et al. \\
\hline & 2010 & Han et al. \\
\hline & 2012 & Aydın et al. \\
\hline & 2015 & Arslan et al. \\
\hline & 2016 & Aydın et al. \\
\hline & 2017 & Hopman et al. \\
\hline
\end{tabular}


booking limits in discrete demand distribution. Brumelle and McGill(1993) used sub-differential optimization to obtain the optimal booking limits both in continuous and discrete demand distribution. Van Ryzin and McGill(2000) proposed a multiple nested fare class model to find the booking limits. Additionally, this method did not need demand forecasting.

Dynamic programming model(DP) relaxed the low to high booking arrival order compared as static model. Lee and Hersh (1993) developed a dynamic single-leg model, which is the first study in DP model. Apart from the above mentioned literature, there are some other academic papers related to dynamic single-leg capacity control, such as Shaykevich(1994), Young and Van Slyke(1994), Brumelle and Walczak (1997),Kleywegt and Papastavrou(1998), Zhao and Zheng(1998),Brumelle and Walczak(1998), Lautenbacher and Stidham (1999), Liang (1999), Subramanian et al. (1999), Zhao (1999) and Talluri and van Ryzin (2004). Among these, Lautenbacher and Stidham prove the monotonicity of the discrete time DP model and unified the dynamic and static model. Liang(1999) propose a continues-time dynamic model and Van Slyke and Young(2000) also give a result of continuous-time version of Lee and Hersh dynamic model. After that, Subramanian et al.(1999) extended the DP model to integrate cancellations, no-shows and overbooking. Apart from the Lee and Hersh DP model, Kleywegt and Papastavrou(1998) proposed a dynamic and stochastic knapsack problem(DSKP). On the base of his own model, they extended DSKP model to incorporate the batch arrivals. Apart from these, there are some recent research on dynamic model, such as Han et al.(2010), Aydın et al.(2012),Arslan et al.(2015), Aydin et al.(2016) and Hopman et al.(2017). A review of these single-leg model can be found in McGill and Van Ryzin(1999, Sec.4), Pak and Piersma(2002,Sec.3) and Talluri and van Ryzin (2006, Chapter $3)$.

Apart from the airline industry, some non-service industries have widely discussed the capacity control problem. Barut and Sridharan (2004) proposed a dynamic capacity allocation procedure model (DCAP) in the make-to-order (MTO) manufacturing environment. The DCAP model dynamically allocates short-term constrained capacity to multiple product classes in multiple periods. Barut and Sridharan (2005) further extended the former DCAP model by relaxing two assumptions. We need to note that there are some similarities and differences between the DCAP model and our model. Obviously, the application of the DCAP model has a focus on the manufacturing industries (especially for make-to-order manufacturing processes). In this case, the demand for each fare class is not necessarily unit-sized. Thus, when a large-sized order for a high value class is rejected, a small-sized order for a low value class can be accepted, given that there is available capacity. Conversely, in our model, a maximum of one customer comes in each time period. When the system rejects a customer 
in a high value class, the revenue will be directly lost. Furthermore, our model considered the multi-channel effect on capacity control in the airline industry, whereas the channel effect is not included in the DCAP models.

2.2 Revenue management with customer-shift behaviour

The earliest study to consider the customer's upward shift was proposed by Belobaba(1989), who analyzed the customer's upward purchasing behaviour in his EMSR model. After that, Weatherford et al.(1993), Brumelle et al.(1990) and Pferifer(1989) took the buy-up factor into consideration. The above model assumes that there are only two fare classes, that is the static models we introduced earlier in the single-leg literature. The specific introduction of the static model will appear in Chapter 4. Based on the static model, You(2001)and You(2003) extended the buy-up factor into the dynamic model. Shumsky and Zhang (2003) studied a cross-time capacity allocation with buy-up factor. Wen(2001) considered the no-show based on the customer buy-up model

The above literature is mainly to discuss the customer's buy-up behaviour under single-leg condition. In addition, there are some literatures that study the influence of customer behaviour on the network model. Andersson(1998) proposed a linear programming model for the network problem considering buyup model. Buke et al(2008) use a stochastic programming approximation to describe the buy-up network problem. Jiang and Miglioico(2014) proposed a dynamic programming network model and use several approximation method to solve it.

The above is some study influence on the customer's buy-up behaviour. Of course there are some revenue management models that take into account the customer's choice behaviour. Instead of using buy-up factor, they use a set of choices, often called choice model. For example, Talluri et al.(2004) proposed the choice model on the base of single-leg model. After that, Liu(2008) extend the choice-based single-leg model to network model and give a approximation method to solve it. After this, there are others who have studied it, like Bront et al.(2009), Kunnumkal(2010), Zhang(2009), Meissner and Strauss(2012),Sierag and van der Mei(2016) and Feldman and Topaloglu(2017). Because this direction is not the main direction of our research, we will not elaborate on it. More specifically, Strauss et al. reviewed the related literature and introduced the theory and model. However, because the choice model is too complex and takes too long to run on actual problems, it is difficult to apply it in the airline's revenue management system. All related literature has been listed in Table 2.2. 
Table 2.2: Customer-shift behaviour literature

\begin{tabular}{c|c|c|c}
\hline Year & Author & year & Author \\
\hline 1989 & Pfeifer & 2003 & You \\
1989 & Belobaba & 2009 & Shumsky and Zhang \\
1990 & Brumelle et al. & 2008 & Büke et al. \\
1993 & Weatherford et al. & 2006 & Cooper et al. \\
1998 & Andersson & 2004 & Talluri and Van Ryzin \\
1999 & McGill and Van Ryzin & 2012 & Cooper and Li \\
2001 & You & 2014 & Jiang and Miglionico \\
2001 & Zhao and Zheng & 2009 & Bront et al. \\
2010 & Kunnumkal and Topaloglu & 2009 & Zhang and Adelman \\
2012 & Meissner and Strauss & 2014 & Talluri \\
2016 & Sierag and van der Mei & 2017 & Feldman and Topaloglu \\
2018 & Strauss et al. & & \\
\hline
\end{tabular}

2.3 Network Revenue Management

Compared with single-leg flight, network revenue management evolved slightly later. With the rapid development of the civil aviation industry, especially the rapid development of the airline's hub-and-spoke network, the research on the network factors of revenue management has become increasingly important. Therefore, our research also integrates channel factors into network revenue management. Below we will review the relevant literature on network revenue management.

In academic literature(see Table 2.3), the earliest research on network revenue problem are Glover et al.(1982), D'Sylva(1982), Wang(1983), Dror et al.(1988). Glover et al.(1982) proposed a deterministic network minimum cost flow model, which is the first study on network effect. Dror et al. extended the deterministic network minimum cost flow model to consider the cancellations. This type of model is also the earliest deterministic linear programming(DLP) model.In addition to the above studies, Wong also studied the DLP model. Wong(1990) proposed a single fare network RM model. On the base of this study, Wong et al.(1993) compared different cabin assignment methods under the single fare case. Additionally, Willamson(1992) discuss the DLP model in extensive simulation studies. Wollmer(1986) developed a linear programming model with stochastic demand, which is the first research on probabilistic 
Table 2.3: Network capacity allocation literature

\begin{tabular}{l|c|c|c}
\hline Year & Author & year & Author \\
\hline 1982 & D'Sylva & 2000 & Kuyumcu and Garcia-Diaz \\
1982 & Glover et al.Glover et al. & 1998 & Chen et al. \\
1983 & Wang & 1999 & McGill and Van Ryzin \\
1986 & Wollmer & 1999 & Ciancimino et al. \\
1988 & Dror et al. & 1999 & Chen et al. \\
1988 & Wysong & 1999 & De Boer et al. \\
1988 & Smith and Penn & 2001 & Feng and Xiao \\
1988 & Williamson & 2002 & Cooper \\
1989 & Simpson & 2001 & Cooper \\
1990 & Wong & 2003 & Bertsimas and Popescu \\
1991 & Phillips et al. & 2003 & Bertsimas and Shioda \\
1992 & Williamson & 2003 & Brumelle and Walczak \\
1990 & Curry & 2004 & Möller et al. \\
1993 & Wong et al. & 2004 & El-Haber and El-Taha \\
1994 & Phillips & 2005 & Bertsimas and De Boer \\
1999 & Talluri and Van Ryzin & 2006 & Klein \\
2008 & Reiman and Wang & 2009 & Zhang and Adelman \\
2009 & Topaloglu & 2012 & Meissner and Strauss \\
2015 & Vossen and Zhang & 2015 & Kirshner and Nediak \\
2017 & Feldman and Topaloglu & & \\
\hline
\end{tabular}


nonlinear programming model(PNLP). Cinacimino et al.(1999) developed a specialized nonlinear programming algorithm to solve the PNLP problem. Chen et al.(1999) combined DLP and PNLP approximations to get the bid-price. Apart from these two approximations, Smith and Penn(1988) first proposed the randomized linear programming approximation method(RLP) and Talluri and van Ryzin(1999) investigated the detail of RLP. The most relevant and closest to the reality yet least reviewed model for network revenue management is the dynamic network model which was proposed by Talluri and Ryzin(1999). Bertsimas and Popescu(2003) extended the proposed algorithm to handle cancellations and no-shows. Klein proposed a concept of self-adjusting bid price to solve the network dynamic programming problem. The detail of dynamic network model and related approximation method also can be found in Talluri and van Ryzin(2006).

The existing literature on the capacity allocation with multi-channel is quite limited in revenue management. Most of the research work on revenue management assume that channel cost is not considered and the airlines directly face to the customers. Hence, some literatures integrated the customer behaviour into the revenue management. Zhang and Cooper(2005) considered customer choice behaviour among parallel flights. Talluri and Van Ryzin(2004) proposed a basic choice-based single-leg revenue management model. Liu and Talluri(2008) developed a approximation method for the network choice-based capacity allocation model, which is called Choice-based deterministic linear programming. And they studied a column generation algorithm to solve it. After that some authors has focused on the choice-based network model, such as Bront et al.(2009), Kunnumkal et al.(2010), Zhang et al. (2009)and Meissner and Strauss(2012) Talluri(2014) and Gallego et al.(2014). As we have said above, it is assumed that the airline directly faces the customer. However, in reality, half proportion of ticket are sold by indirect channels and every airlines pay a lot of commission fee to indirect channel every year. Therefore, the behaviour of the channel is often more important than the behaviour of the customer. However, most of the literature on network revenue management ignored the channel's effect on airlines' revenue. Additionally, some researches on other aspect of network revenue management, such asTopaloglu(2009), Vossen and Zhang(2015) and Kirshner and Nediak(2015). A review of these network literatures can be found in McGill and Van Ryzin(1999), Pak and Piersma(2002), Chiang et al.(2006) and Talluri and Van Ryzin(2006). We also review the detail of dynamic network model(Bertsimas and Popescu,2003) and some approximations method which related in our research in Chapter 5. 
Before the advent of e-commerce, research on channels was rare, especially in the aviation industry. Because the original channel of the civil aviation industry mainly distributes resources to various travel agencies or ticket agents through the GDS system. Due to the fact that civil aviation's own channels are affected by the cost, market share is not large, such as call centres and outlet stores. With the rapid development of e-commerce, the transparency and convenience brought by online ticketing have made it quickly become the main channels for customers. At the same time, it also gives airlines the opportunity to face customers directly, such as building up direct sales websites and launching direct sales Apps(Buhalis and O'Connor,2006). The Internet-based sales mode brings opportunities and challenges to airlines. Below the last part of the literature review is some research on multi-channel issues, which has been mentioned widely in traditional manufacturing products marketing literature since the rapid development of e-commerce.

In airlines, multi-channel distribution has been implemented for many years(see Table 2.4), but only few studies have focused on the effect of multichannel distribution on airlines revenues. Jarach (2002) showed that internetbased ticketing in airlines would bring some changes for airline companies through the analysis of the impact on the e-commerce-oriented airline. Toh and Raven(2003) was the first to focus on the impact of the channel on the airline's revenue and suggested that the Integrated Internet marketing concept (IIM) wanted to integrate inventory, prices, markets, and channels into one system. However, they just put forward an idea and conducted a channel impact analysis, and did not propose a specific model. Shon et al. (2003) proposed that online channels would dominate the tickets market compared to traditional channels. Alamdari and Mason (2006) demonstrated that changes were taking place in airline distribution and predicated that the direct channels would substantially increase in the airlines. Through an empirical survey on customers, Yoon et al. (2006) discovered that the airlines' e-commerce activity might have important effects on their ticket distribution channels. Castillo-Manzano, et al. (2010) analysed the customer choice behaviour in purchasing air tickets from the traditional channel and online channel. In our paper, we will also discuss the impacts of airlines operations management on air ticket distribution channels. Ruiz-Mafe et al. (2009) and Bigne et al. (2010) studied the motivation and barriers for customers to purchase tickets on websites. Koo et al.(2011) proposed a decision support model to analysis the relationship between the direct channel and indirect channel like online travel agency(OTA). They found that when the customers had loyalty, the airline liked to use direct channel rather than OTA. Otherwise, the OTAs have competitive. Wei Yihua(2008) has discussed the 
Table 2.4: Multi-Channel literature

\begin{tabular}{|c|c|c|}
\hline Objective & Year & Author \\
\hline \multirow{13}{*}{ Airline } & 2002 & Jarach \\
\hline & 2003 & Shon et al. \\
\hline & 2003 & Toh and Raven \\
\hline & 2004 & Hornick \\
\hline & 2006 & Alamdari and Mason \\
\hline & 2006 & Yoon et al. \\
\hline & 2005 & Buhalis and O'Connor \\
\hline & 2008 & Wei Yihua \\
\hline & 2009 & Ruiz-Mafe et al. \\
\hline & 2010 & Castillo-Manzano and López-Valpuesta \\
\hline & 2010 & Bigné et al. \\
\hline & 2011 & Koo et al. \\
\hline & 2001 & Smith et al. \\
\hline \multirow{2}{*}{ Hotel } & 2002 & Choi and Kimes \\
\hline & 2005 & Rao and Smith \\
\hline \multirow{7}{*}{ Manufacturer } & 2003 & Chiang et al. \\
\hline & 2003 & Yao and Liu \\
\hline & 2004 & Wallace et al. \\
\hline & 2005 & Alptekinoğlu and Tang \\
\hline & 2004 & Barut and Sridharan \\
\hline & 2005 & Barut and Sridharan \\
\hline & 2014 & Xie et al. \\
\hline Marketing & 2005 & Rangaswamy and Van Bruggen \\
\hline \multirow{2}{*}{ Overview } & 2004 & Simchi-Levi et al. \\
\hline & 2008 & Agatz et al. \\
\hline Railway & 2014 & Cheng and Huang \\
\hline Tourism & 2015 & Fountoulaki et al. \\
\hline
\end{tabular}


pricing problem for revenue management with multiple channel. Apart from the airline industry, some studies have also investigated same channel distribution issues in similar industries such as hotels and high speed rails. Choi and Kimes (2002) conducted some simulations to testify the effects of electronic distributions on hotel revenue management. Rao and Smith(2005) use decision support model to hotel channel distribution. Cheng and Huang (2014) adopted an empirical survey for Taiwan high speed rail industry to identify the ticketing channel preference for customers. Fountoulaki et al. (2015) reported the new channel distribution for travel and tourism using on the case of Crete.

Compared with perishable asset industries(like airline, hotel and railway), channel sales in manufacturing are also affected by e-commerce. Therefore, there are also some manufacturing documents that we can refer to. Chiang et al.(2003) study the issue of multi-channel design. They found that direct sales channels will bring more profits to manufacturers. After that, Yao and Liu(2003) and Wallace et al.(2004) both study the effect of direct channel on traditional channel and prove that the direct channel will make the market system stable under some conditions. Xie et al.(2014) proposed a model integrate the capacity allocation and channel distribution in traditional manufacturing system. An overview of manufacturing multi-channel literature can be found in Simchi-Levi et al. (2004)

The detail of literature on multi-channel also can be found in Agatz et al.(2008), Boyd and Bilegan(2003).

\subsection{Summary}

Since the 1970s, academic research on revenue management has been going on for more than 40 years. With the development of the civil aviation market and the development of computer technology, the revenue management model has evolved from the original static two-class single-leg model to the current dynamic multi-class network model. At the same time, the study of revenue management has a profound influence on the industry, so it is also generally taken seriously by the industry. However, when we see that in the Internet era, more and more manufacturing companies have undergone transformation and upgrading to adapt to the development of the Internet such as online and offline coordination. As an airline core system, the revenue management system still continues the characteristics of the previous century and does not keep up with the development of the Internet age. Because of this, many professional managers are eager to have a new revenue management system[52] and academic researchers need to integrate Internet characteristics into a revenue management system to provide guidance for practice managers through empirical and model 
studies. However, through the above literature review, we find that there are some deficiencies in the current theoretical research in this area:

(1) There is a lack of analysis of channel costs in the revenue management literature. There may be two reasons. First, the basic assumption of revenue management is to consider revenue without considering costs. Because both civil aviation and hotels, their fixed costs far exceed the variable costs, so it is usually assumed that every seat are only considered revenue. However, with the rapid development of civil aviation industry and increased competition, especially the emergence of low-cost carrier, more and more airlines are paying attention to variable costs. The cost of the channel accounts for most of the variable cost of the airline, because the airline pays a part of the agency fee for each ticket sold through the agency channel(Toh and Raven,2003). The second reason is that the original civil aviation channels are almost monopolized. All the tickets are distributed to the agents through GDS, and the agents sold the products to customers. Airlines do not have better channels to face customers directly, so they usually choose to ignore this part of management. Therefore, we found that almost all the revenue management literature, whether it is single-leg or network capacity allocation, did not discuss the issue of channel cost. However, the rapid development of Internet channels has caused the above two reasons to disappear. At the same time, it also gives airlines the opportunity to face customers through direct channels. As a result, airlines need to integrate channels into company management and develop new revenue management systems.

Therefore, the research of this paper is mainly to combine channel management and revenue management. Through the overall balance of channel costs and airline ticket revenues, total airline revenue has been increased.

(2) There is a lack of analysis of customer choice behaviour when they face the channel in the literature. Most of the revenue management literature assume that the customer is directly facing the airlines. However, the study of the airline market chain shows that more than half of flight tickets are sold by different channels. Therefore, customer behaviour in the channel side greatly influences the airline's control of the channel.

Therefore, this study analysis the research on the channel side and proposes customer behaviour with channel transfer behaviour. On this basis, we have improved the original channel revenue management model to improve the application of the model.

(3) The airline channel distribution literature lacks applicability and operability. Although there is literature investigating the airline channel distribution, such studies seldom coordinate the revenue management system. Most of the airline channel distribution literature recognizes that the distribution costs need to be reduced for airlines, but few of them propose detailed solutions to achieve this. 
Therefore, Our research focuses on channel allocation and management at the operational levels. We combined the inventory control model with the channel control and dynamically control the capacity within the channel and the fare class in each time period to achieve the purpose of reducing channel costs and increasing revenue.

(4)Because traditional network models usually is complex and hard to solve, they lack integrated design and research of channel issues. The traditional network model also needs to use the approximation method to solve, such as DLP, PNLP, RLP and other approximation models. If you integrate the channel control problem, the model may be more complicated. But as we have said before, if the channel issues are not integrated with the revenue management issues, then each individual decision may bring losses to the airlines.

Therefore, our research propose a dynamic network capacity allocation model which integrating multi-channel factors. Additionally, we applied a linear programming model to approximate it and make the problem easy to solve. That make the model more applicable. 
3 Dynamic capacity allocation for single-leg with Multi-Channel distribution

\subsection{Single-leg capacity allocation model}

In this section, we will first introduce traditional single leg capacity control and booking control policies. Normally, single leg capacity control model has two stream: static solution method and dynamic solution methods. The biggest distinguish between static models and dynamic models is the customer arrival process. Static single leg solution methods assume that the customer arrives in order of increasing fare class(See figure 3.1). For instance, low value customer arrives before high value customer.

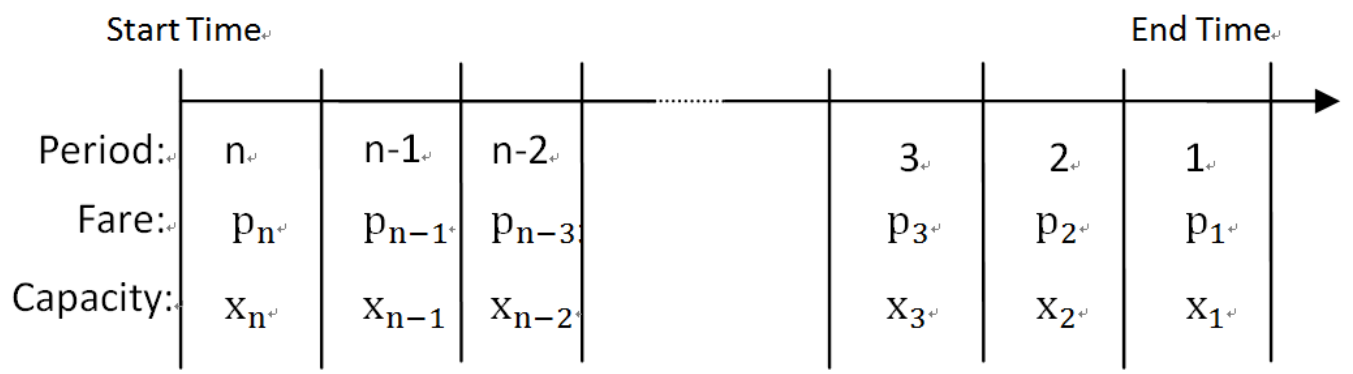

Figure 3.1: Static solution booking process.

In contrast, dynamic solution methods assume that the booking requests is not in order of increasing fare class. At any moment, customers with different needs have a chance to arrive. The dynamic system decides whether to accept the customer through the marginal price.(See figure 3.2) The static solution methods are described in section 3.1.1 and the dynamic solution methods are described in section 3.1.2

\subsubsection{Traditional Static Model For Single-leg Capacity allocation}

In this section, we will introduce the static model for single-leg, which is the first developed and applied in airline industry. The earliest single-leg capacity control research is due to Littlewood(Littlewood,1972). Before RM model building, Littlewood proposed six assumptions for their static model.

- The customer for different fare classes arrives in nonoverlapping periods in the order of increasing fare classes. 


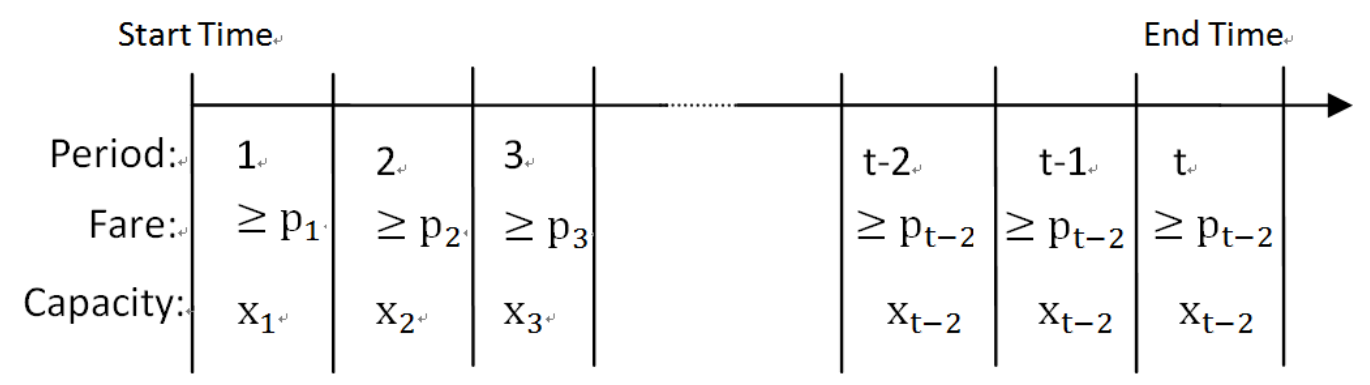

Figure 3.2: Dynamic solution booking process

- The demands for different fare classes are independent random variables.

- The demand for a given class will not be affected by the inventory control.

- The static models ignore some details about the control process in every periods. for example, in practical, demand may not come in a order over time and sometimes comes in a batch in a period.

- The static models do not concern the group booking request.

- The static models assume risk-neutrality.

Basing on those assumptions, some static solution methods are modelled. Next we will introduce some static model. Firstly, we introduce the earliest single leg capacity control model: Littlewood's two-class model. The model has two fare classes in a single leg flight. The total flight seats is $\mathrm{S}$ and the two class' fare is $f_{1}>f_{2}$. The demand for class 1 is denoted $D_{1}$ and its distribution is denoted by $\varepsilon_{1}(x) \cdot \operatorname{Pr}\left(D_{1} x\right)$ represents the probability of selling all protected seats $x$ to high fare class demand. $f_{1} \operatorname{Pr}\left(D_{1} x\right)$ is the marginal value, which decides whether to accept low fare class or not. The model denotes $x$ as the remaining seats. The process is described in figure3.3. At first, a customer for low fare class comes. If the marginal value with remaining seats $x$ is lower than the low fare class fare, the airline will accept the low fare class and receive the revenues of $f_{2}$. Otherwise, if the marginal value with remaining seats $x$ is higher than the low fare class fare, the airline will reject low fare class and close the low fare class. After that, the airline only accept the high fare class.

Note that the expected value is related to remaining seats $x$ and decreasing in $x$. Hence, we can find a optimal remaining seats to simply the model. When the remaining seats $x$ is less than the optimal points, the airlines will reject the low fare class. This optimal points is denoted as protection level, $\tau_{1}$. Therefore, the protection level $\tau_{1}$ must satisfy the condition below:

$$
f_{2}<f_{1} \operatorname{Pr}\left(D_{1} \tau_{1}\right) \text { and } f_{2} f_{1} \operatorname{Pr}\left(D_{1} \tau_{1}+1\right)
$$

This is called Littlewood's rule. The advantages of Littlewood's model is simple and easy to implement. Airlines only need to use a demand distribution 


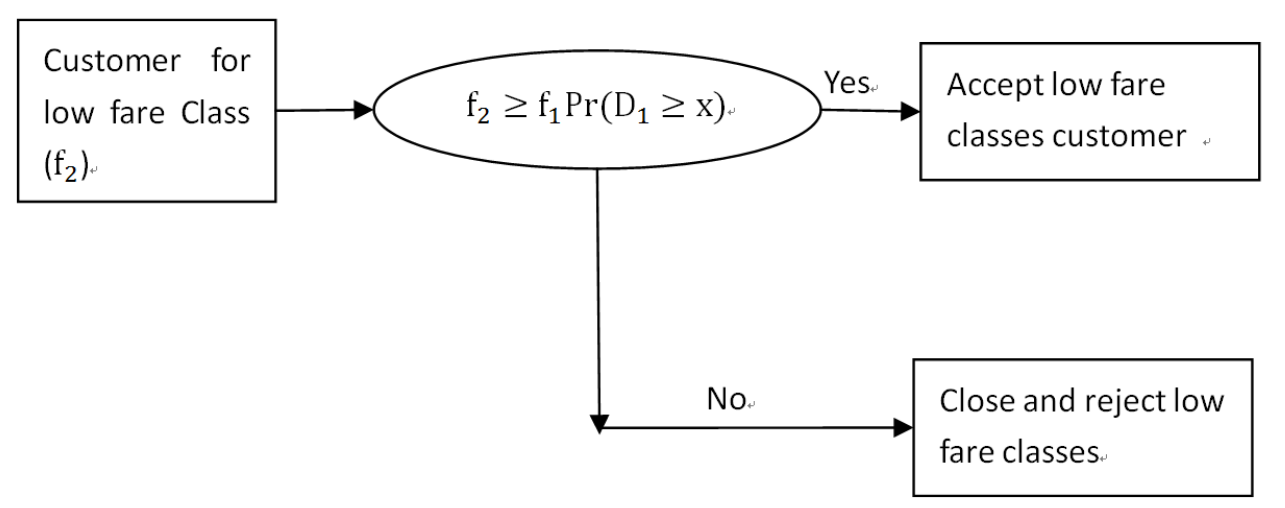

Figure 3.3: Littlewood's model booking process

and then the capacity in different fare class can be calculated. However, the disadvantages of Littlewood's model is also obvious. In reality, customer will not arrive in order of fare class. Therefore, Littlewood's model is not in line with the actual situation. As we mentioned before, the static model has several assumptions. Most of assumptions make the model far from the reality. Therefore, in the history of revenue management research, Scholars are constantly relaxing these assumptions to get closer to reality.

The littlewood model is limited to a two-class model. On this basis, many scholars have proposed multiple classes. The multi-level problem can be described as the airlines divide the tickets into different prices to sell for different customer groups. For example, as the figure 3.4 showing, the tickets from Shanghai to Frankfurt have several fare classes in the China Eastern Airline websites. According to the examples, we can see that airlines sell the multiple fare classes in the reality. Therefore, capacity allocation need to consider the multiple fare classes problems.

Here we introduce multiple fare class model. The airlines sell one flight seat in a increasing order fare classes. The fare class order can be noted as $f_{1}>$ $f_{2}>\ldots>f_{n}$. The booking process in multiple fare class model is almost the same as the two-class model. In the first period, the airline only opens the lowest fare class and accepts lowest fare class (like fare $f_{n}$ )request. And in the next period, the airline closes the above fare class $f_{n}$ and opens the fare class $f_{n-1}$, and so on, until the last period $f_{1}$. It's hard to solve this problem by twoclass model. Generally, the techniques for finding the optimal booking limits 


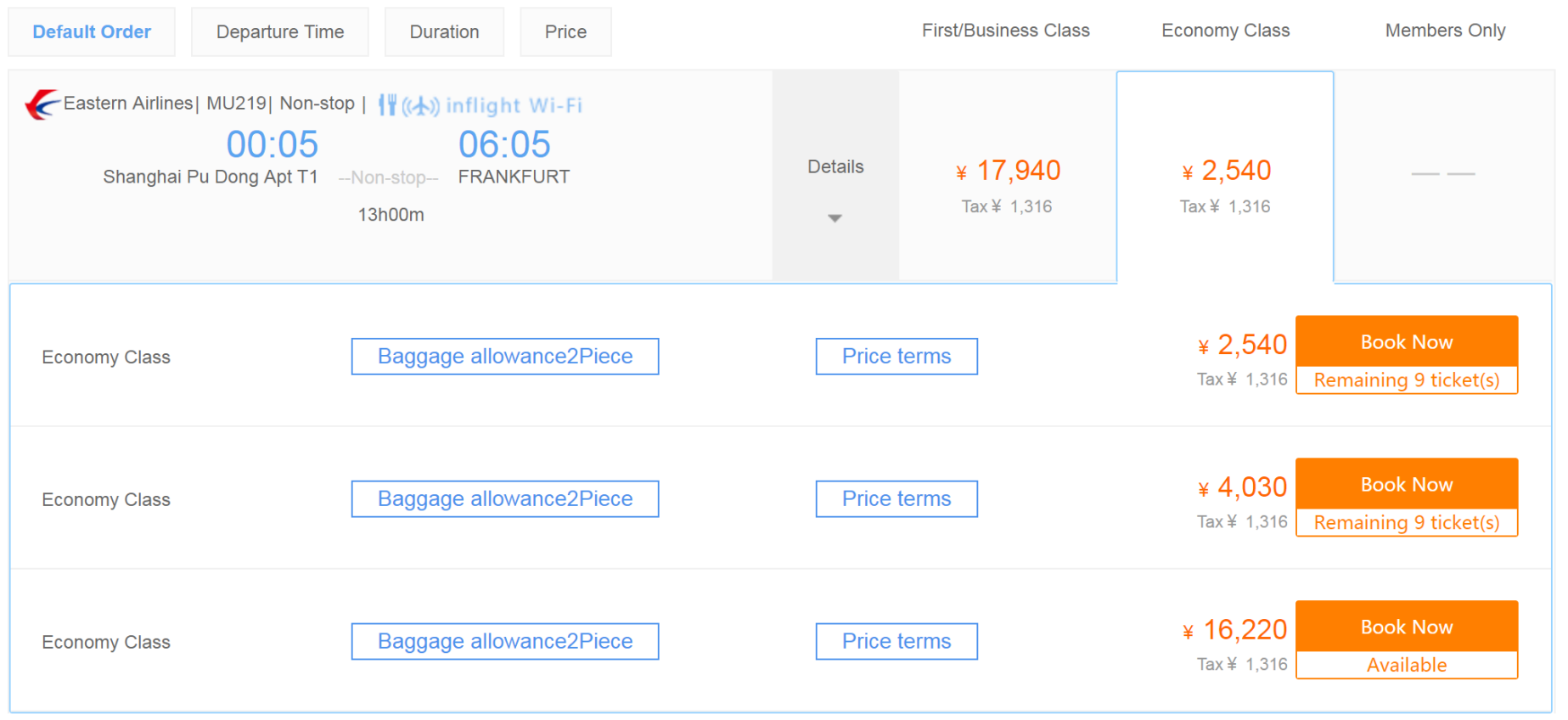

Figure 3.4: Fare class from Shanghai to Frankfurt

for three or more fare classes require the use of dynamic programming. As the assumptions, the bellman function is :

$$
r_{i}(x)=E\left[\max _{0 \leq \varphi \geq \min \left\{d_{i}, x\right\}}\left\{f_{i} \varphi+r_{i-1}(x-\varphi)\right\}\right]
$$

With boundary conditions:

$$
r_{0}(x)=0, x=0,1, \ldots, C .
$$

$C$ is the total capacity. $\varphi$ is quantity of the customer which is accepted by airlines in each fare class(optimal control seats). After solving the dynamic programming, the model can get optimal booking limit in every fare class. Although the dynamic programming model for finding the optimal booking limits in the Multiple class model seems not mathematically difficult from today's view, at 1980s the airline choose some easy heuristics to solve n-class model in their revenue management system. The most widely used heuristics is the expected marginal seat revenue(EMSR) approaches which is developed by Belobaba(1987).

The EMSR approaches estimates multi-class problems through a series of Littlewood's two-class models. The solution process is to calculate sum of protection level of current class relative to its higher by using Littlwood's rule. Here we illustrate a small example that use EMSR approach. A flight has three 
fare classes. If we want to know the protection level of class 3 , first we need to calculate the protection level for class 3 against class 1 by using equation (3.1):

$$
\tau_{31}=\operatorname{Pr}_{1}^{-1}\left(\left(f_{1}-f_{3}\right) / f_{1}\right)
$$

and the protection level for class 3 agains class 2 :

$$
\tau_{32}=P r_{2}^{-1}\left(\left(f_{2}-f_{3}\right) / f_{2}\right)
$$

Therefore ,the protection level for class 3 is the sum of these two protection levels, which is:

$$
\tau_{3}=\tau_{31}+\tau_{32}=\operatorname{Pr}_{1}^{-1}\left(\left(f_{1}-f_{3}\right) / f_{1}\right)+\operatorname{Pr}_{2}^{-1}\left(\left(f_{2}-f_{3}\right) / f_{2}\right)
$$

According to the above example, the researcher proposed generalized model as follow:

$$
\tau_{j}=\sum_{i=1}^{j-1} \operatorname{Pr}_{i}^{-} 1\left(f_{i}-f_{j} / f_{i}\right)
$$

According to the EMSR approach above, the airlines can easily deal with the multiple class model. More precisely, the EMSR approach is same as the Littlewood model when the flight only have two fare classes. However, the EMSR model still does not break the limitations of the static model. Although closer to reality than the littlewood model, the problem of multi-level models was discussed.

\subsubsection{Traditional dynamic Model for single-leg}

This section will introduce the dynamic model for single-leg, which is relevant to our DCAM model. The dynamic model for single leg can be found in Talluri and van Ryzin(2006). Compared with the static model, the dynamic model no longer assigns customers with different values to the specified time period. In the dynamic model, customers with different values arrive randomly at each time period. Due to the different arrival rates of customers with different values in reality, the dynamic model usually gives different value customers an arrival probability to simulate the airline ticketing process. The key contribution of DP model is relaxing the low to high revenue order(low fare customer comes first and then high fare customer comes) assumption. Other static model assumptions 
are retained as we mentioned in the section 3.1.1. The demand for different fare classes are independent random variables. And the airlines are assumed as risk-neutral.

The model assumptions and model formulation is described below:

$$
r_{t}(x)=E\left[\max _{\omega \in(0,1)}\left\{\operatorname{Prod}(t) \omega+r_{t+1}(x-\omega)\right\}\right]
$$

With boundary conditions:

$$
\begin{aligned}
& r_{T}(x)=0 \quad \forall 0 \leq x \leq N \\
& r_{t}(0)=0 \quad \forall 0 \leq t \leq T
\end{aligned}
$$

The DP model assumes that one single-leg has $N$ seats. $\operatorname{Prod}(t)$ is the revenue made in time $t$ periods. If a customer for class $j$ comes in time period $t, \operatorname{Prod}(t)=p_{j}$, otherwise $\operatorname{Prod}(t)=0 . p_{j}$ is the fare of class $j$. The remaining capacity is denoted as $x$. The purpose of the model is to maximize the revenue of a flight. The decision to make this revenue is controlled by binary variable $\omega$. When the customer is accepted by the airlines, $\omega=1$ and otherwise $\omega=0$.

As we mentioned before, DP model relaxed the simple Low-to-high order assumptions. Additionally this makes the DP model closer to reality because in the reality you can not imagine all demand arrives in your order, most of time is disorderly. Because of the different policies of the static model, the dynamic model needs new policies to solve the problem. As our DCAM model in section 3.2 has some relations with the DP model, next we will introduce the related policies of the single-leg model.

\subsubsection{Control policy for single leg}

The control policies or control mechanisms are important in implementation level of revenue management for airlines. Among them, there are three main control strategies, namely booking limits, protection level and bid price.

Booking limits and Protection level

The control policy of booking limits is one of the basic and applicable control mechanisms. Booking limits are defined that each class has a capacity limit at a given point in time.(Defined in Talluri, 2006) Protection level is a similar control for an amount of capacity to protect for each class in a flight. Protection 
level and booking limits are relative concepts. Protection level is to protect the capacity of the high fare class. In contrast, booking limit is to limit the capacity of the low fare class. Both of these two controls can be partitioned and nested. According to the figure 3.5, we specify partitioned booking limits, partitioned protection level, nested booking limits and nested protection level.

Class 1

$¥ 16220$,
Class 2

$¥ 4030$ 。
Class 3

$¥ 2540$ 。

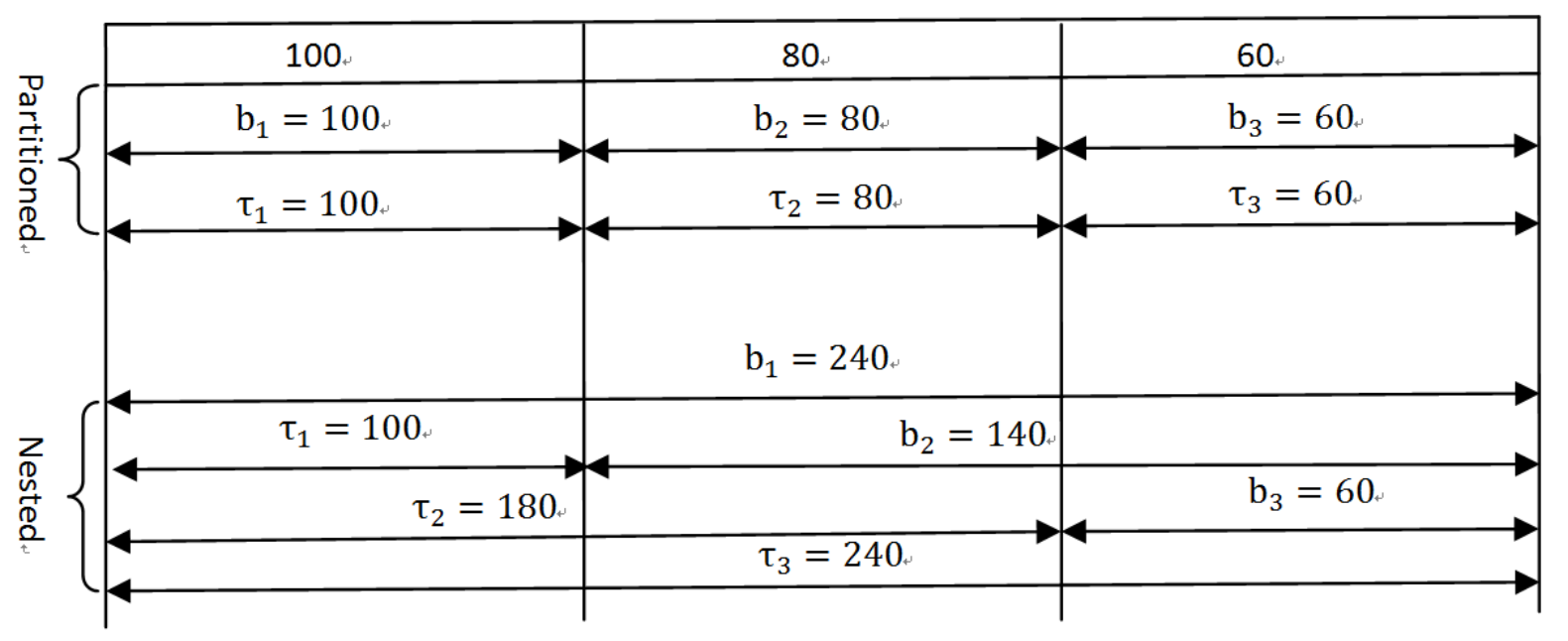

Figure 3.5: Partitioned and Nested booking limits and protection level

We use the flight data from eastern airline website (See figure 3.4) to explain these four control policies. A partitioned booking limits distribute the available capacity into determined class. Here, we denote $b_{c}$ as the booking limits of fare class $c$, Which $c \in 1,2,3$. And we also denote $\tau_{c}$ as the protection level of class $c$. When one class demand is over than its booking limit, this class will be close. As the figure 3.5 showed, the airlines set a partitioned booking limit of 100 seats for class 1,80 seats for class 2 and 60 seats for class 3 . When 100 seats are sold in class 1 , the class 1 will be close. At this time, low revenues class which are not sold out are still open. It can be seen from this point that it is not reasonable by using partitioned booking limit. As the denotation of protection level, we can see from the figure 3.5 that the protection level of each class is the same as the booking limit in the partitioned control.

Compared the partitioned control policy, nested control policy seems more reasonable. We use the same data to explain that. A nested booking limit is demonstrated that the higher revenue class can access to the capacity of lower revenue class after the capacity of the higher revenue class used up. For example(See figure 3.5), with the total capacity of 240, the booking limit of high revenue class(Class 1 ) is 240 . That means that the customer for class 1 can buy the seat until all seats are sold out. The booking limit of class 2 is 140 . That 
means the customer for class 2 can buy any seat in class 2 and class 3 but the airline has to reserve 100 seats for class 1 . The booking limit of class 3 is 60 which means that the class 3 is closed after the capacity of class 3 is sold out.

As the denotation, the nested protection level is different from the nested booking limit. As shown in figure 3.5 , the protection level is equal to $C-b_{j}$. $C$ is defined as total capacity and $b_{j}$ is defined as the booking limit of class $j$. For example, the protection level of class 1 is 100 . It means that the airline must leave 100 seats for class 1 in a flight. Additionally, the airline will leave 180 for class 1 and class 2 . At this time, class 3 can not be sold. And the protection level $\tau_{3}$ means that in this case all the class is opened for any customers.

\section{Bid Price}

The bid price control is simply to control the opening of the fare class through the marginal price. We use the previous example to illustrate how to apply bid price control. There are three fare classes that the fare is $f_{1}=16220$, $f_{2}=4030, f_{3}=2540$ respectively. The time period is $T=100$. When time $t=70$, the marginal price is 5200 which is higher than $f_{2}$ and lower than $f_{1}$. The demand request from class 2 and class 3 will be rejected and the demand request from class 1 will be accepted. And When time period $t=30$, the marginal price is 3000 which is higher than $f_{3}$ and lower than $f_{2}$. The demand request from class 3 will be rejected and the demand request from class 1 and class 2 will be accepted. The bid price control and booking limits control are different. The bid price control is dependent on the price and the booking limits control is dependent on the fare-class. The bid price control is usually used in dynamic programming capacity allocation model. In our model, we mainly use bid price control.

\subsubsection{Summary}

In summary, after introducing the traditional single-leg capacity allocation model, we know that the traditional model focused on the capacity allocation in different fare class for achieving the maximum revenues. Compared with the static model, dynamic programming model is closed to the reality but not easy to solve. Therefore, some study introduced bid price optimal control to solve it. However, none of these capacity allocation model considered the channel effect. In the next part, we will proposed our new DP model which integrated channel effect into revenue management system. On the base of the new model, we also propose optimal policy to solve it. And finally we design some experiments to test the model and propose some implications for airline company according to the results. 
3.2 Dynamic capacity allocation for airlines with multi-channel distribution

\subsubsection{Problem definition}

The dynamic capacity allocation with multi-channel distribution can be described as the following problem(DCAMP). Assume an airline company selling $N$ identical products(seats) for a single leg flight. Products are divided by $\mathrm{n}$ fare classes and each fare class has its price $p_{j}$, where $j=\{1, \ldots, n\}$. The products are sold by $i=\{0, \ldots, m\}$ channels. Let channel 0 be the direct channel and channel $1, \ldots, m$ as different indirect channels. There are $t=0, \ldots, T$ time periods in a finite selling horizon. Each time period is small enough such that maximum one customer will arrive in one period. Time starts from $t=0$ and ends at $t=T$. In each period, the customer arrives at $i$ channel and require $j$ class with probability $\lambda_{i j}$. It holds that $\lambda_{0}+\sum_{i=1}^{m} \sum_{j=1}^{n} \lambda_{i j}=1$, where $\lambda_{0}$ is the probability that no customer arrives at that time period. When one seat is sold by channel $i$, the airline pays $\delta_{i} p_{j}$ commission fee to channel $i$. At the beginning of each period, when one demand arrives in one channel requiring one fare class, the airline company firstly decides whether to accept the channel request or not. This decision is denoted $w_{i} t$ coded to one if the airline company accepts the channel request and 0 otherwise. Then, the airline company makes the second decision about fare class request. The decision is again denoted by a binary variable $u_{j t}$. If $u_{j t}=1$, the airline company accepts this fare request and 0 otherwise. After the customer request has been accepted, the airline company obtains a revenue $\left(1-\delta_{i}\right) R_{j}(t)$, or otherwise $\left(1-\delta_{i}\right) R_{j}(t)=0$ in case of no arrival or request rejected. The decision depends on the remaining capacity $x$, each time period $t$, the channel revenues rate $\delta_{i}$ and the fare class $p_{j}$, with the objective to maximize the total expected revenues in a selling horizon from the airline company's perspective.

Now, we can present the main mathematical programming formulation with the state variable remaining capacity $x$, the channel decision variable $w$, the product decision variable $u$ and the stage variable $t$ to describe the airline decision process. The model develops a dynamic airline seat booking capacity allocation model in Lee and Hersh (1993), Lautenbacher and Stidham (1999), and Liang (1999). Basing on their model, we integrate the channel factor into airline dynamic seat booking capactiy allocation model and analyse the effect in different situation. The premise of our model is to have the authority to limit the capacity of each channel. Based on this premise, our model integrates the capacity allocation for fare class and the capacity allocation for the channel into a new dynamic model. We also discuss the optimal policy for the new model. 


\subsubsection{Dynamic capacity allocation with a multi-channel model}

We define the following parameters that will be used throughout the paper.

Parameters:

$i: \in\{0, \ldots, m\}$ represents every channel in a single-leg flight.

$j: \in\{0, \ldots, n\}$ represents the every fare class in a single-leg flight.

$t$ : represents each time period.

$T$ : finite horizon periods.

$N$ : totally identical products in one leg flight

$w_{i t}$ : the channel decision binary indicator such that $w_{i} t=1$ if the airline company accepts channel $i$ 's request in time period $t$, and 0 otherwise.

$u_{j t}$ : the fare class decision binary indicator such that $u_{j t}=1$ if the airline company accepts fare class $j$ request in time period $t$, and 0 otherwise.

$\delta_{i}$ : channel $i$ 's commission fee rate.

$R_{j}(t)$ : revenues made from fare class $j$ in time period $t$.

$x$ : the integer variable representing the number of products left (capacity).

$V_{t}(x)$ : the maximum expected revenues of the remaining capacity $x$ in period $t$.

Based on the parameters, the Bellman equation is then written as:

$$
V_{t}(x)=E\left[\max _{w_{i t} \in(0,1), u_{j t} \in(0,1)}\left\{w_{i t}\left(1-\delta_{t}\right) u_{j t} R_{j}(t)+V_{t+1}\left(x-w_{i t} u_{j t}\right)\right\}\right]
$$

With boundary conditions:

$$
\begin{aligned}
& V_{T}(x)=0 \quad \forall 0 \leq x \leq N \\
& V_{t}(0)=0 \quad \forall 0 \leq t \leq T
\end{aligned}
$$

Boundary condition (3.12) will be applied when the last period $T$ comes, and the airline company will make no decision after period $T$, regardless of the remaining capacity. Boundary condition (3.13) will be applied regardless of the time period. When the remaining capacity becomes 0 , the expected revenues will be 0 .

\subsubsection{Optimal control policy for DCAM}

We optimize the model and find the optimal control policy. For analysing the optimal control in this case, (3.11) can be rewritten as the following equation: 


$$
\begin{array}{r}
V_{t}(x)=V_{t+1}(x)+E\left[\max _{w_{i t} \in(0,1), u_{j t} \in(0,1)}\left\{w_{i t}\left(1-\delta_{t}\right) u_{j t} R_{j}(t)+V_{t+1}(x-1)-V_{t+1}(x)\right\}\right] \\
=V_{t+1}(x)+\sum_{i=1}^{m} \sum_{j=1}^{n} \lambda_{i j}(t)\left[\left(\left(1-\delta_{i}\right) p_{j}+V_{t+1}(x-1)-V_{t+1}(x)\right)_{+}\right]
\end{array}
$$

With the same boundary conditions.

We define $\Delta V_{t+1}(x)=V_{t+1}(x)-V_{t+1}(x-1)$ as the expected marginal value (EMV) of the capacity at time period t, which is the expected increasing value of the $x_{t} h$ unit of the capacity. The optimal control policy is based on EMV. When EMV is lower than the revenues from channel $i$ in fare class $j$ (we assume that the revenue is only related with fare price $p_{j}$ and the commission rate $\delta_{i}$, which is $\left.\left(1-\delta_{i}\right) R_{j}(t)=\left(1-\delta_{i}\right) p_{j}\right)$, the airline company accepts the request, and otherwise, it rejects it. The EMV is calculated by function (3.14) and is related to state variable $x$ and time period $t$. Therefore, the optimal condition for accepting channel $i$ in class $j$ can be described as $\left(1-\delta_{i}\right) p_{j} \geq \Delta V_{t+1}(x)=$ $V_{t+1}(x)-V_{t+1}(x-1)$. The optimal control can use the bid price where we define the bid price as the following equation.

$$
\pi_{t}(x)=\Delta V_{t}(x)
$$

When revenues exceed the bid price, the seat request is accepted and otherwise is rejected. Based on the definition of value function (3.11), $V_{t}(x)$ and the definition of EMV $\Delta V_{t+1}(x)$, we can obviously obtain the following important properties:

Lemma 1 For $t=0,1, \ldots, T, V_{t}(x)$ is concave and non-increasing in $x=$ $0,1, \ldots, N$.

Lemma 2 The expected value of capacity $\Delta V_{t}(x)$ is non-increasing with state variable $x$ and time period $t$.

$$
\begin{aligned}
\Delta V_{t}(x+1) & \leq \Delta V_{t}(x) \\
\Delta V_{t+1}(x) & \leq \Delta V_{t}(x) \quad \forall 0 \leq x \leq N, \forall 0 \leq t \leq T
\end{aligned}
$$

Proof of Lemma (2): we first introduce a concave function definition and a lemma proven by Stidham (1978).

Definition 1 A function $g: \mathbb{Z}_{+} \rightarrow \mathbb{R}$ is concave if and only if $g(s)-g(s+1)$ is non-increasing in $s$.

Lemma 3 Suppose $f: \mathbb{Z}_{+} \rightarrow \mathbb{R}$ is concave. Let $g: \mathbb{Z}_{+} \rightarrow \mathbb{R}$ be defined by

$$
g(x)=\max _{b=0,1, \ldots, n}\{b p+f(x-b)\}
$$


Then, for any $p \geq 0$ and non-negative integer $n, g(x)$ is concave in $x \geq 0$.

The proof of Lemma (1) is based on (3), and we prove it by induction on t. $V_{T}(x)=0$ for all $x$. Therefore, $V_{T}(x)=0$ is trivially concave and nonincreasing. Assume that $V_{t+1}(x)$ is concave and non-increasing, and let $g(x)=$ $\max \left\{\delta_{i} p_{j}+V_{t+1}(x), V_{t+1}(x-1)\right\}$. Then,

$$
V_{t}(x)=V_{t+1}(x)+\sum_{i=1}^{m} \sum_{j=1}^{n} \lambda_{i j}(t) g(x)
$$

According to Lemma (3), $\mathrm{g}(\mathrm{x})$ can be easily proven to be concave. Because $\sum_{i=1}^{m} \sum_{j=1}^{n} \lambda_{i j}(t)$ can be regarded as a non-negative constant, $\sum_{i=1}^{m} \sum_{j=1}^{n} \lambda_{i j}(t) g(x)$ is also concave. $V_{t+1}(x)$ is concave as we have assumed. Thus, based on the principle that the sum of two concave (nonincreasing)functions is also a concave function, we can prove that $V_{t}(x)$ is also a concave and non-increasing with respect to $x$.

Proof of Lemma (2): because the value function $V_{t}(x)$ is concave, the increments $\Delta V_{t}(x)$ are non-increasing in $x$.

To prove the monotonicity of $\Delta V_{t}(x)$ in $t$, we have:

$$
\begin{aligned}
& \Delta V_{t}(x)=V_{t}(x)-V_{t}(x-1) \\
& =V_{t+1}(x)+E\left[\max _{w_{i t} \in(0,1), u_{j t} \in(0,1)}\left\{w_{i t} \delta_{i} u_{j t} R_{j}(t)-\Delta V_{t+1}(x)\right\}\right] \\
& -\left\{V_{t+1}(x-1)+E\left[\max _{w_{i t} \in(0,1), u_{j t} \in(0,1)}\left\{w_{i t} \delta_{i} u_{j t} R_{j}(t)-\Delta V_{t+1}(x-1)\right\}\right]\right. \\
& =\Delta V_{t+1}(x)+E\left[\max _{w_{i t} \in(0,1), u_{j t} \in(0,1)}\left\{w_{i t} \delta_{i} u_{j t} R_{j}(t)-\Delta V_{t+1}(x)\right\}\right. \\
& \left.-\max _{w_{i t} \in(0,1), u_{j t} \in(0,1)}\left\{w_{i t} \delta_{i} u_{j t} R_{j}(t)-\Delta V_{t+1}(x-1)\right\}\right]
\end{aligned}
$$

Because of $\Delta V_{t}(x) \leq \Delta V_{t}(x-1)$ as just proven,

$$
E\left[\max _{w_{i t} \in(0,1), u_{j t} \in(0,1)}\left\{w_{i t} \delta_{i} u_{j t} R_{j}(t)-\Delta V_{t+1}(x)\right\}\right] \geq
$$




$$
E\left[\max _{w_{i t} \in(0,1), u_{j t} \in(0,1)}\left\{w_{i t} \delta_{i} u_{j t} R_{j}(t)-\Delta V_{t+1}(x-1)\right\}\right]
$$

This is therefore larger than or equal to $\Delta V_{t}(x)$.

These two Lemmas demonstrate the monotonicity of $\Delta V_{t}(x)$ with respect to time period $t$ and capacity $x$. The monotonicity $\Delta V_{t}(x)$ implies that for one deterministic time period $t$, for each channel $i$ and each fare class $j$, there exists a critical capacity point $\hat{x}_{i j}(t)$ that a request for a seat in channel $i$ and fare $j$ is denied for the remaining capacities $x<\hat{x}_{i j}(t)$ and accepted for $x \geq \hat{x}_{i j}(t)$. With the remaining capacity $x$ given, for each channel $i$ and each fare class $j$, there also exists a critical time period point $\hat{t}_{i j}(x)$ that a demand for a seat in channel $i$ and fare class $j$ is denied for $t<\hat{t}_{i j}$ and accepted for $t \geq \hat{t}_{i j}(x)$. Obviously, from the above two implications, we can imply that for a time period $t$, remaining capacity $x$ and a determined class $j$, there exists a set of channel $\hat{i}_{j}(t, x)$ that the demand for a seat in channel $i$ and fare class $j$ is denied for $i_{j}>\hat{i}_{j}(t, x)$ and accepted for $i_{j} \leq \hat{i}_{j}(t, x)$.

These implications show how the airline makes the decision (deny/accept) with the information of the time period, remaining capacity, class and channel by using our optimal policy when demand comes. Therefore, the optimal policy developed here provides theoretical support in the following sections (4) for analysing the channel-shift customer behaviours.

\subsection{Numerical experiment and analysis}

\subsubsection{Design of experiments}

In this section, we design several experiments for examining our model in different situations. Our model describes the integration of channel choice and fare choice and the relationship between the channel distribution system and revenue management system. Thus, there will be a number of problems to be investigated. We have illustrated some representative examples Table 3.1 to demonstrate the characteristics of our model and the outcomes when decisions are integrated.

Experiment 1 examines the relationship between the commission fee and airline company revenues in multichannel and single-fare classes. Customer shift behaviour is not considered in this experiment. Experiment 2 examines the change of airline company revenues with different numbers of channels in one fare class. We assume that there is one demand pattern, one determined commission fee and no customer shift behaviour. Experiment 3 discovers the differences between the classic revenue dynamic model and our multichannel model in the multi-fare system. We assume that each channel has the same market 
Table 3.1: Experiment list

\begin{tabular}{c|cccc}
\hline Experiments & No.of Channels & No.of Fare Classes & Demand pattern & Commission Fee Case \\
\hline 1 & 2 & 1 & 1 & 3 \\
2 & $2 \& 3$ & 1 & 1 & 1 \\
3 & $1 \& 2$ & 2 & 1 & 1 \\
4 & 2 & 1 & 3 & 1 \\
\hline
\end{tabular}

share and that each fare class has the same arrival rate. In addition, these experiments have the same length of time. The fare and the channel commission rates are all the same. Experiment 4 investigates how our multichannel system behaves when facing different demand patterns (i.e., with high and low demand volumes). Among these experiments, we include the classic single-leg dynamic programming model (DP in section 3.1.2, classic system, see Lee and Hersh, 1993) as the benchmark for comparison purposes. More specifically, the DP model is an independent fare class capacity allocation model, and the channel part in the reality will depend on the rule of first come, first serve.

\subsubsection{The effect of commission fee on airlines revenue}

In the first experiment, we discuss the effects of commission rates on airline revenues and channel allocation by implementing our policy. We proposed the following examples as in Table 3.2 for running our numerical experiment. An airline has two channels: Channel A is indirect (like GDS) and Channel D is direct (like airline websites). These two channels differ in terms of their market shares (in our model, market share can also be replaced by arrival rates) and commission rates. For explicitly describing the result of channel allocation, the example only has one fare class.

Table 3.2: Channel Characteristics for Experiment 1

\begin{tabular}{c|ccc}
\hline Channel & Market Share & Fare Class(Fare) & Commission Rate \\
\hline A(CH.A $)$ & $74 \%$ & 1 class(260) & $13 \%, 23 \%, 33 \%$ \\
D(CH.D) & $26 \%$ & 1 class(260) & $0 \%, 0 \%, 0 \%$ \\
\hline
\end{tabular}


The experiment flight leg has 50 capacities, and the time period for selling is $\mathrm{T}=100$. Through 1,000 runs of the simulator for different commission rates, the experiments have different maximum revenues that are averaged over 1,000 runs, and the results are shown in Table 3.3. In this experiment, we compare the total revenues using the DP model and the DCAM model, the customer proportions in channel $\mathrm{A}$ and channel $\mathrm{D}$, and the increasing percentage of revenues from the DP model to the DCAM model. As illustrated in Table 3.3, the DCAM model system makes more revenues than the classic system through reallocating the capacity using the fare class and channel. Even at the low commission rate of 0.13, the DCAM improves revenues by $3.99 \%$ compared to the DP model.

Moreover, we analyse the sources of the improvements and the sales behaviours in the time periods. From the results of allocating seats in CH.A and CH.D (Table 3.3), the seat sold by CH.D has improved by $53.76 \%$ compared to the original $74 \%$ (See Table 3.2), and the seat sold by CH.A has decreased to $46.24 \%$. More seats allocated to CH.D (direct channel/low cost channel) improve airline revenues. However, according to Figure 3.6, CH.A is not useless in all time periods. At the beginning, CH.A sells more seats than the CH.D because of the high volume of demand, and then our optimal policy limits CH.A and relaxes the restriction to CH.D. The policy lets CH.D receive more seats than CH.A in the middle range of selling periods. In the latter periods, CH.A meets much more demand than the CH.D and increases the volume of sales. In general, our model does not simply restrict the allocation of seats to CH.A and shift the seats to CH.D, but rather, it compromises the demand volume, channel costs and the time of demand to reallocate the seats for CH.A and CH.D.

Table 3.3: Results of Experiment 3.3.2

\begin{tabular}{c|ccccc}
\hline Commission rate & Revenues for DP & Revenues for DCAM & Seat allocation in CH.A & Seat allocation in CH.D & Inc.rate \\
\hline 0.13 & 11749.4 & 12218.5 & $46.24 \%$ & $53.76 \%$ & $3.99 \%$ \\
0.23 & 10787.4 & 11647.3 & $45.24 \%$ & $54.76 \%$ & $7.97 \%$ \\
0.33 & 9825.4 & 11052.3 & $47.47 \%$ & $52.04 \%$ & $12.48 \%$ \\
\hline
\end{tabular}

If we consider the effect of commission rate, the total revenues will decrease with the increasing commission rate, either by the DP model or the DCAM model. However, in this case, with the comparison of the DP and DCAM models, revenues will decrease more slowly in DCAM (Figure 3.7).

Thus, this experiment concludes the following:

- The DCAM model improves revenues over different commission rates compared with the DP model. 


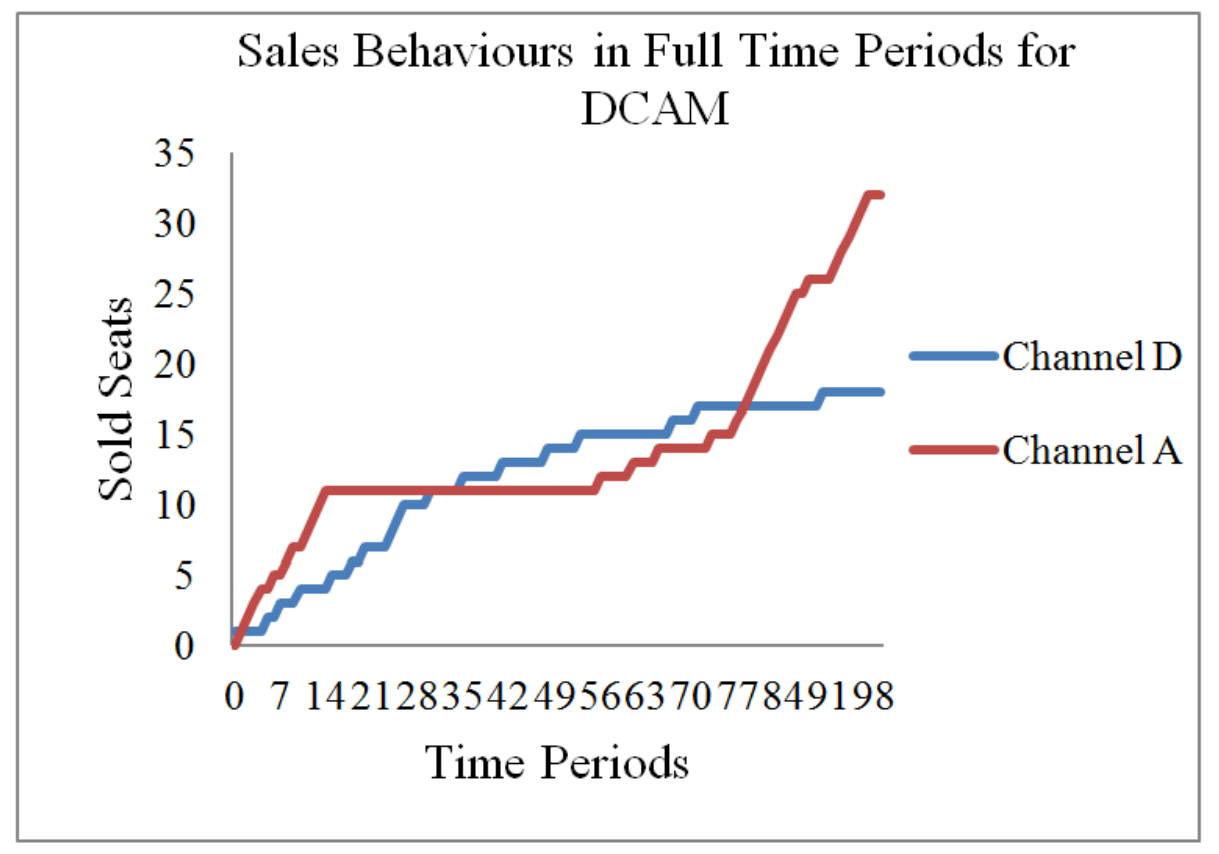

Figure 3.6: Sales Behaviours in Experiment 3.3.2

- The DCAM model does not simply limit the indirect channel and increase the seats in the direct channel, but rather, it compromises the demand volume, channel costs and time of demand to dynamically reallocate the seats for all channels.

- The DCAM model can reduce the lost revenues due to increased commission fees compared to the DP model.

\subsubsection{The effect of channel amount}

In the second experiment, we discuss whether the number of channel affects the revenues by using the DCAM model or not. The experiment adopts the double channel example and the triple channel example with one fare class $(\mathrm{p}=260)$. The selling horizon is divided into $\mathrm{T}=100$ time periods, and the total capacity is 50. The double channel example has two channels: Channel A (as Agency) has an $82 \%$ market share with a $19 \%$ commission rate, and Channel D (as airlines website) has an $18 \%$ market share with a $0 \%$ commission rate. For the triple channel case, we consider two situations. Firstly, we add one additional low commission rate channel (Low CR), and then we add one additional high commission rate channel (High CR). This consideration can fully verify the effect with the consideration of the number of channels. We assume that Channel A and Channel $\mathrm{B}$ are different travel agencies with different arrival rates and 


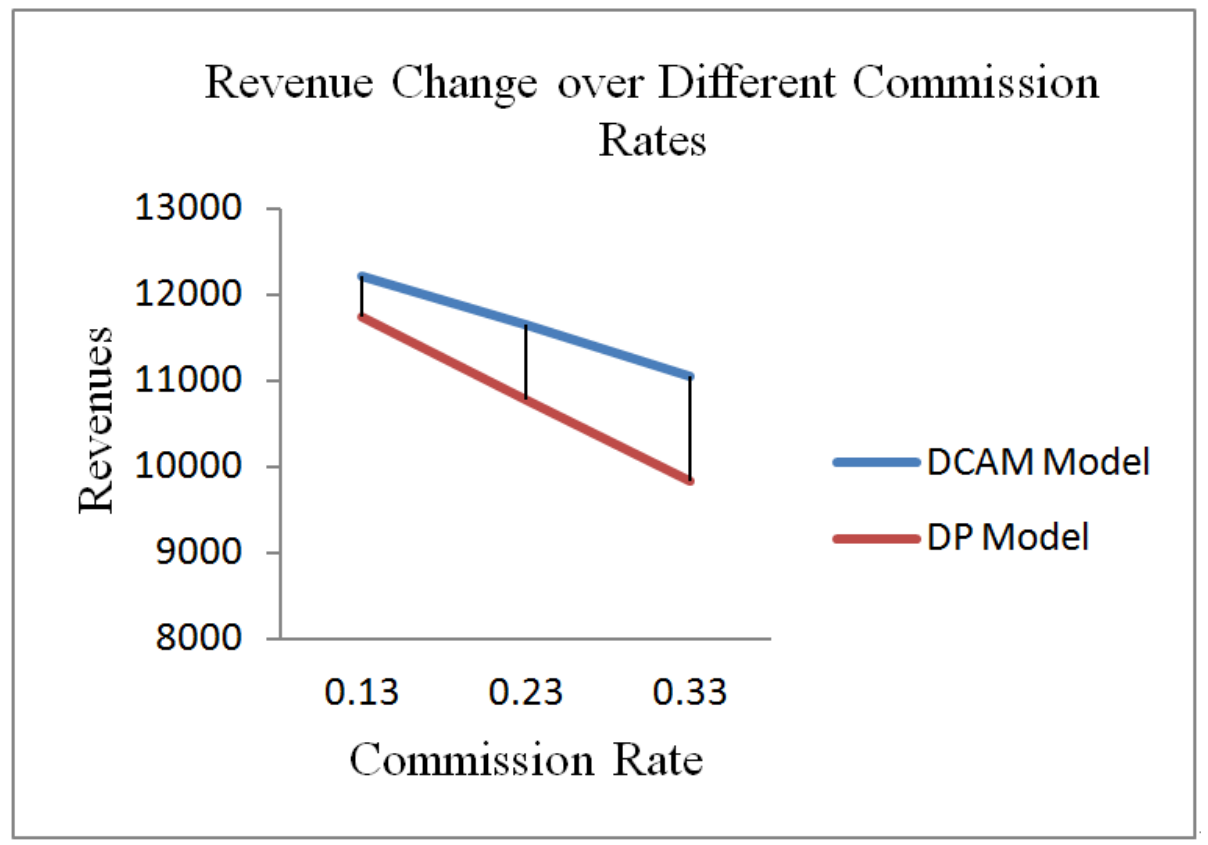

Figure 3.7: Revenue Changes over Different Commission Rates

different commission rates. The experiment is simulated 1,000 times, and we assess the average results.

Table 3.4: Results of Experiment 3.3.3

\begin{tabular}{c|cc|cccc|ccc}
\hline & \multicolumn{3}{|c}{ Double Channel } & \multicolumn{2}{c}{ Triple Channel(Low CR) } & \multicolumn{2}{c}{ Triple Channel(High CR) } \\
\hline & CH.A & CH.D & CH.A & CH.B & CH.D & CH.A & CH.B & CH.D \\
Arrival Rate & 0.82 & 0.18 & 0.51 & 0.31 & 0.18 & 0.51 & 0.31 & 0.18 \\
Commission rate & $19 \%$ & $0 \%$ & $19 \%$ & $9 \%$ & $0 \%$ & $29 \%$ & $19 \%$ & $0 \%$ \\
Seat allocation & $62.55 \%$ & $37.45 \%$ & $6.54 \%$ & $58.93 \%$ & $34.53 \%$ & $6.81 \%$ & $59.46 \%$ & $33.73 \%$ \\
\hline Rev.DP & 10974.6 & & 11377.6 & & 10311.6 & \\
Rev.DCAM & 11455.3 & & 12149.8 & & 11282.1 & \\
Inc.rate & $4.37 \%$ & & $6.78 \%$ & & & & \\
\hline
\end{tabular}

As illustrated in Table 3.4, the DCAM system improves revenues in both the double channel distribution system, and the triple channel system compared to classic system. More precisely, the triple channel with the low CR added improves revenues by $6.78 \%$ compared to the DP model, and the improvement is also higher than double channel. Compared with the absolute revenues of the double channel, the triple channel with the low CP is an improvement, whereas the triple channel with the high CR has lower revenues than the double channel. Nevertheless, the improvement from the DP to the DCAM is the highest in the 
case of the triple channel with the high $\mathrm{CR}$. Where do the improvements come from? Additionally, why does this happen? We plot the sales behaviours for the full periods for the explanation (Figure 3.8) of the case of the triple channel with the high CR. From the analysis of sales behaviours, at the beginning and middle stages of the sales period, almost all seats are sold by Channel B and Channel D. In the latter periods, Channel A sells more seats because Channel $\mathrm{B}$ and Channel D have already fulfilled their demand. Therefore, regardless of adding one low $\mathrm{CR}$ channel or one high $\mathrm{CR}$ channel, the increasing rate between the DP and the DCAM improves because the airlines receive more requests from relatively low $\mathrm{CR}$ channels and rejects more requests from relatively high $\mathrm{CR}$ channels, although it has a high arrival probability. On the other hand, in the DP model, airlines receive requests only by first come first serve principle, which means that it only depends on arrival probability. In conclusion, the DCAM model can efficiently allocate the capacity in the multi-channel system (regardless of adding the high $\mathrm{CR}$ channel or the low $\mathrm{CR}$ channel) to maximize the airline's revenues.

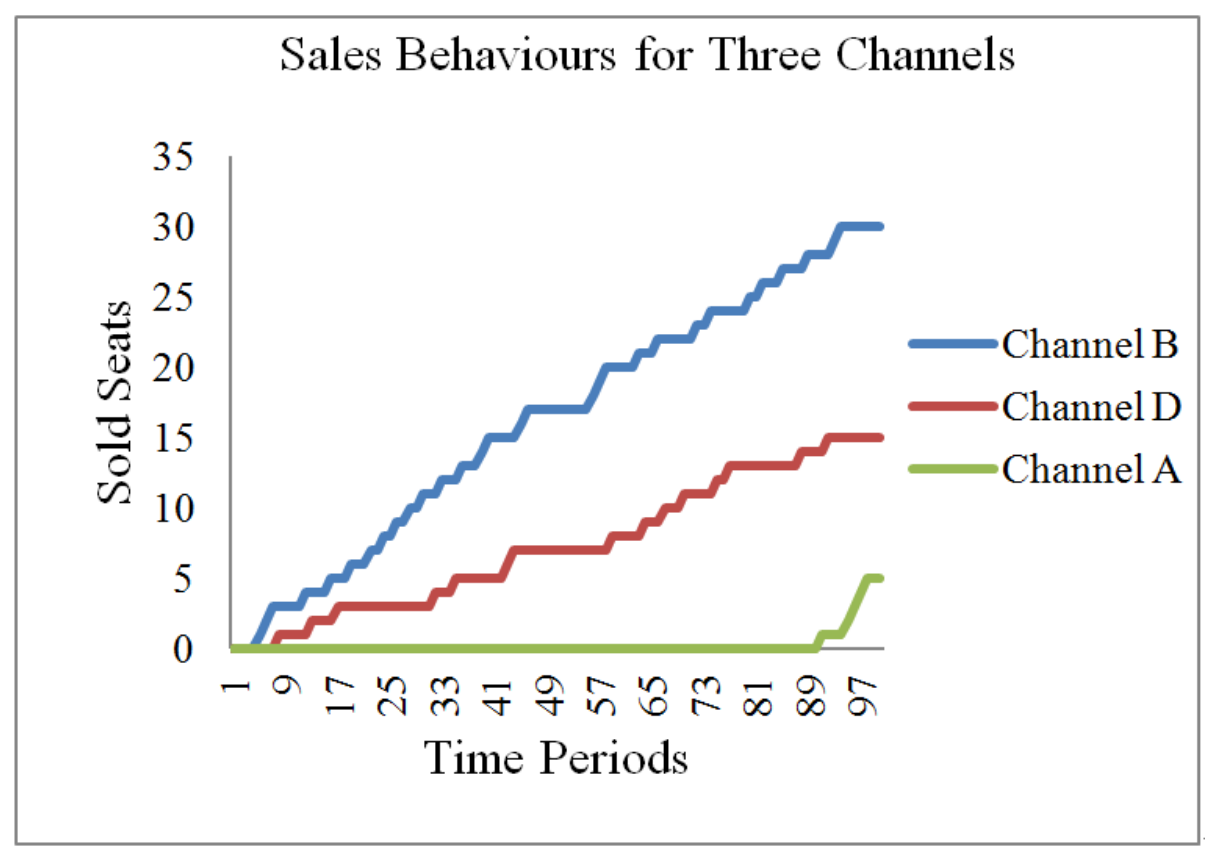

Figure 3.8: Sales Behaviour in Experiment 3.3.3

\subsubsection{Dynamic channel distribution with two fare classes and double channels}

Experiment 3 examines the efficiency of the DCAM model in the multi-fare system. We assume that a single-leg flight has two different classes of customers with fare prices $\left\{p_{1}, p_{2}\right\}=\{260,200\}$ and arrival rates with probabil- 
ities $\left\{\lambda_{1}, \lambda_{2}\right\}=\{0.24,0.75\}$. The airline sells these tickets in two channels with the commission fee rates $\left\{\delta_{1}, \delta_{2}\right\}=\{0.13,0\}$ and customer arrival rates in channels with probabilities $\left\{\lambda_{a}, \lambda_{b}\right\}=\{0.74,0.26\}$. The selling horizon is also divided into $T=100$ periods, and the total capacity is 50 . As Table 3.5 illustrates, the DCAM model can reallocate the capacity to customers both in the fare classes and channels, which does not follow the first come, first serve order. The DP model can only reallocate the fare classes, and the channel allocation applies first come, first serve order. Therefore, the experiment results demonstrate that the DCAM model improves revenues by $3.15 \%$ compared to the DP model.

Table 3.5: Results of Experiment 3.3.4

\begin{tabular}{c|ccccc}
\hline & Channel A & Channel D & Fare class 1 & Fare class 2 \\
\hline Arrival Rate & 0.74 & 0.26 & 0.24 & 0.75 \\
Commission rate & $13 \%$ & $0 \%$ & Price & 260 & 200 \\
Seat allocation by DCAM & $53.12 \%$ & $46.88 \%$ & & $68.05 \%$ & $31.95 \%$ \\
Seat allocation by DP & $74 \%$ & $26 \%$ & $68.79 \%$ & $31.21 \%$ \\
\hline Rev.DP & \multicolumn{5}{c}{10903.2} \\
Rev.DCAM & \multicolumn{5}{c}{11247.4} \\
Inc.rate & \multicolumn{5}{c}{$3.15 \%$} \\
\hline
\end{tabular}

For more precise analysis, we adopt one experiment for our DCAM system and observe the sales behaviours in all time periods (Figure 3.9). We supposed that aircraft seats are sold in 4 situations, including the high commission fee channel with the high fare class (CHAF1), the high commission fee channel with the low fare class (CHAF2), the low commission fee channel with the low high fare class (CHDF2) and the low commission fee channel with the high fare class (CHDF1). From Figure 3.9, we have the obvious result that CHAF1 is the best seller among these four situations. However, CHAF1 does not have the highest volume (the highest volume belongs to CHAF2, which means that CHAF2 has a high arrival rate) and does not have the highest net revenue per seat sold (the highest value is CHDF1). Thus, the result of our optimal choice has relationships with the airlines' revenues and the customer volume of each channel and fare. This also explains why at the end of periods, the high-volume situation CHAF2 sells more seats, as seen in Figure 3.10.

When we compare the DCAM system with multi-fare and the DCAM system without multi-fares, we find some differences. The increasing rate of the DCAM with multi-fares is lower (3.15\% vs. 3.99\%). By observing the sales behaviours in Figure 3.10, we see that such a reduction of improvement in our experiment 


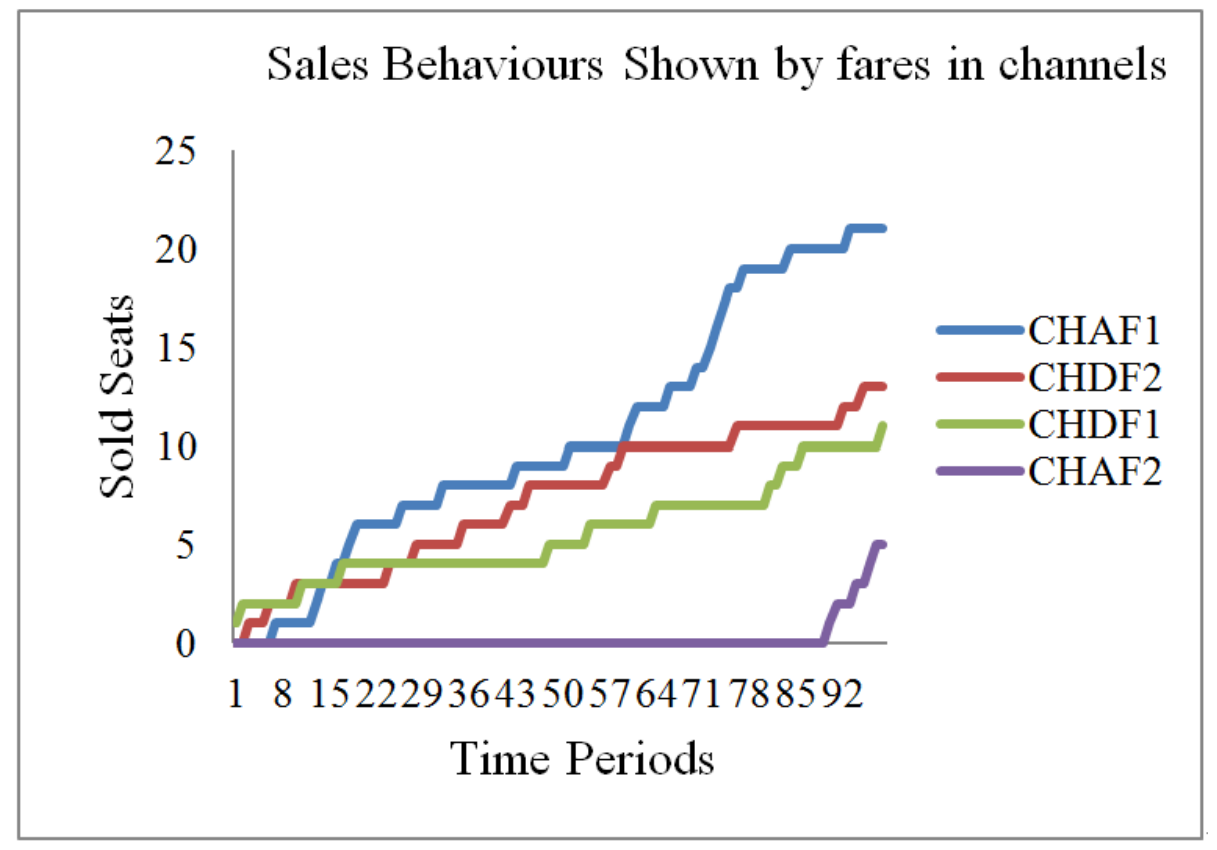

Figure 3.9: Sales Behaviours Shown by Fares in Channels

could be due to the assumption of having a maximum one customer in one period (i.e., when the customer is rejected in the period, demand is lost). For example, when a customer is rejected by the high fare class with a high commission fee channel, this high fare demand is lost, and in the next period, the seat probably is sold by the low fare class with a low commission fee channel. Hence, the whole system will lose the opportunity of revenue improvement compared with the multi-channel system. In conclusion, the integration of the multi-channel and multi-fare system still has a good performance in our experiment.

\subsubsection{Channel allocation with different demand patterns}

Experiment 4 compares the performances of the classic system and the DCAM system over different selling horizons and with different capacities. In the example, we assume that the customer arrival rate for each time is 0.99 , which represents that there is a $99 \%$ possibility that one customer will come in each time period. Thus, the time period can be roughly viewed as the value of total demand. This experiment adopts the basic parameters of experiment 3.3.2 (Table 3.2). The total capacity for each example is 50, and the time period is $30,100,300$, which represents the low demand, middle demand and high demand volumes, respectively.

Table (3.6) illustrates that for the low demand case, there are less revenue differences between the DP and DCAM models. The result confirms the reality in air tickets sales. When the airline does not have sufficient customers, it 


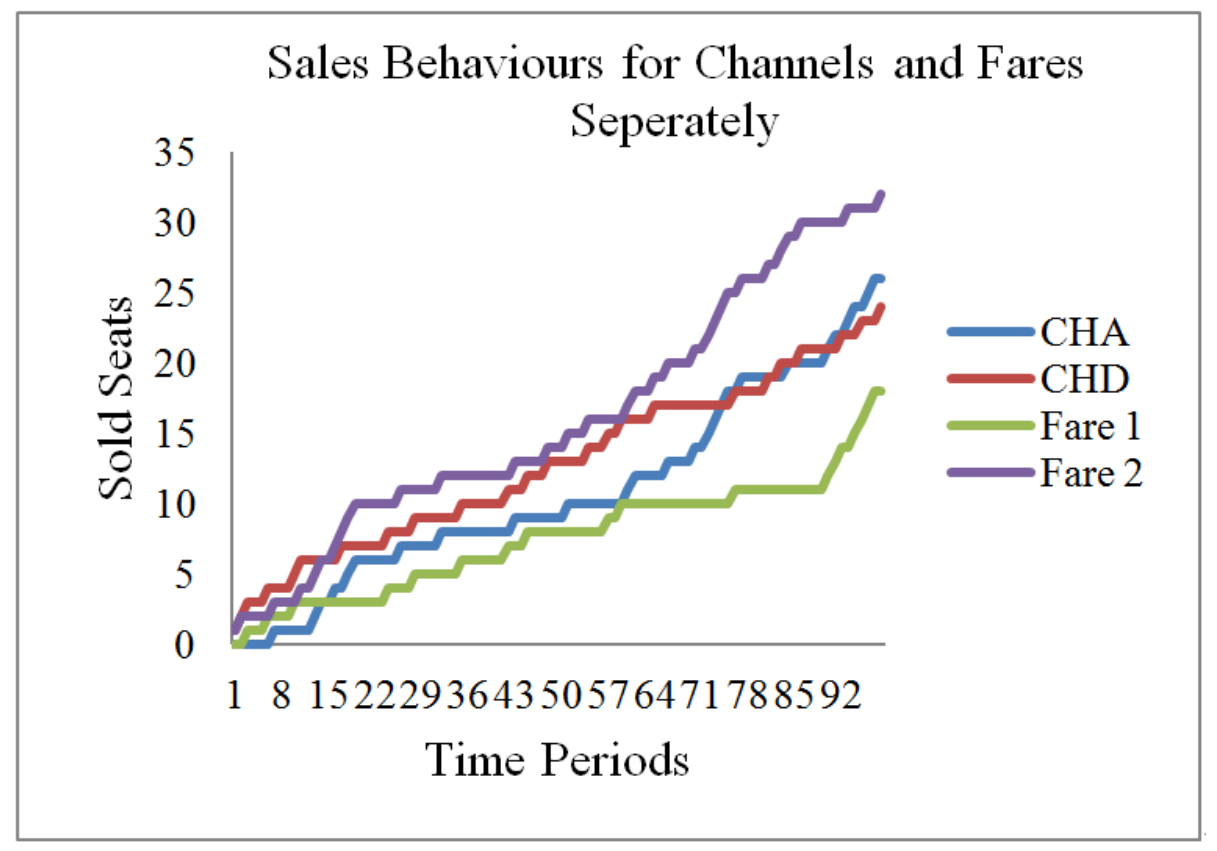

Figure 3.10: Sales Behaviours for Channels and Fares Separately

Table 3.6: Results of Experiment 3.3.5

\begin{tabular}{c|cccccc}
\hline Time & Capacity & Revenues for DP & Revenues for DCAM & Seat allocation of CH.A & Seat allocation of CH.D & Inc.rate \\
\hline 30 & 50 & 7049.64 & 7033 & 0.5004 & 0.0996 & $-0.23 \%$ \\
100 & 50 & 11749.4 & 12218.5 & 0.4624 & 0.5376 & $3.99 \%$ \\
200 & 50 & 11749.4 & 12958.3 & 0.0244 & 0.9756 & $10.64 \%$ \\
\hline
\end{tabular}


attempts to sell every ticket to every coming customer to increase its revenues. This sales behaviour can also be shown in Figure 3.11. Therefore, when low demand happens, the optimal channel allocation for the DCAM completely depends on the arrival rate of each channel. The second case presents that normal demand volume provides more revenue improvements by using the DCAM system rather than by using the classic system. The customer proportion of each channel will be reallocated by the DCAM model.

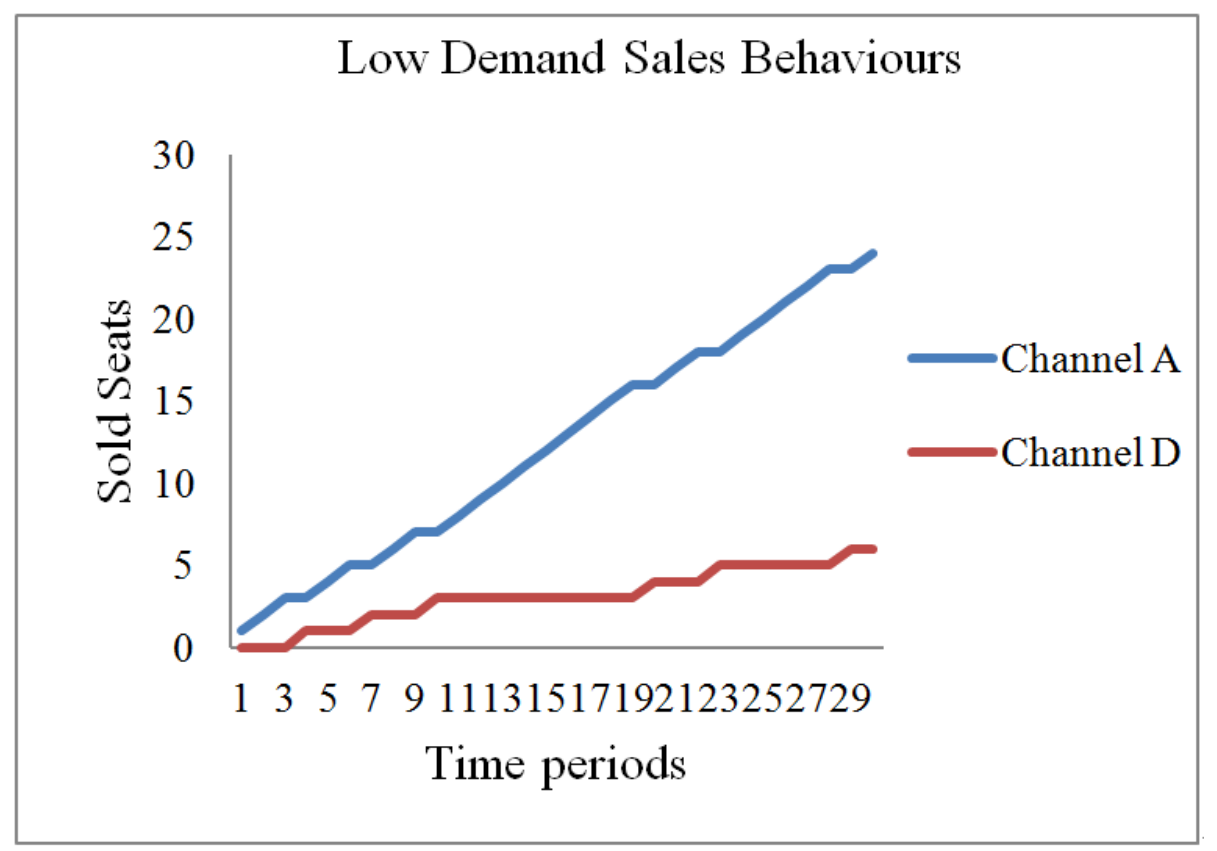

Figure 3.11: Low Demand Pattern Sales Behaviours

Figure 3.12 demonstrates how the DCAM system controls the capacity to improve the revenues. As mentioned in the above experiments, in the beginning periods, the system sells more seats through channel $\mathrm{D}$, and at the end of periods, the system sells more seats through Channel A because of its high arrival rate.

When the extremely high demand occurs (Figure 3.13), the channel control system will choose all the low commission fee channels such as the direct channel (CH.D), and the DCAM model makes the highest revenue improvements. These three cases imply that demand forecasting for the DCAM system is important and will influence the revenue improvements.

3.4 Conclusion

In this Chapter, we have studied the problem of capacity control in the singleleg flight fare class and multi-channel distribution. We proposed a dynamic programming model for integrating the fare class capacity allocation and the 


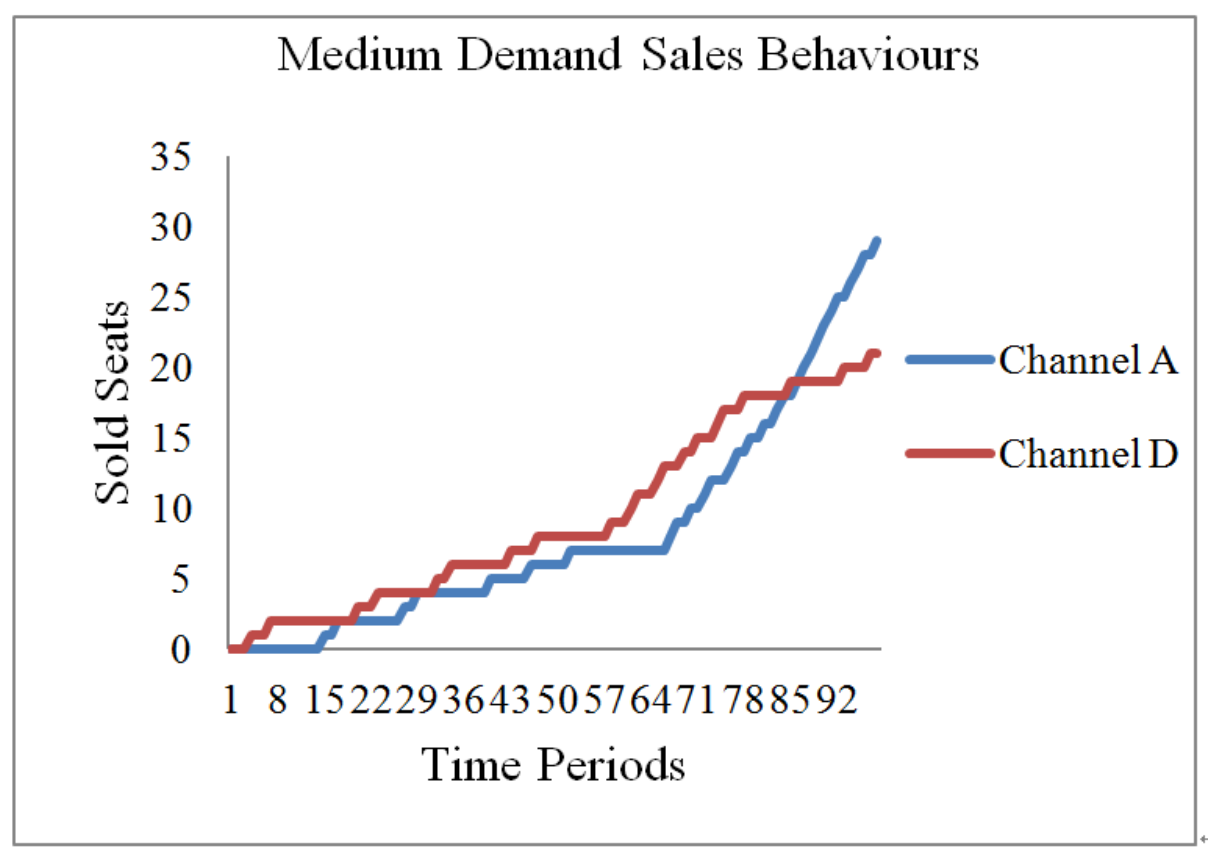

Figure 3.12: Middle Demand Pattern Sales Behaviours

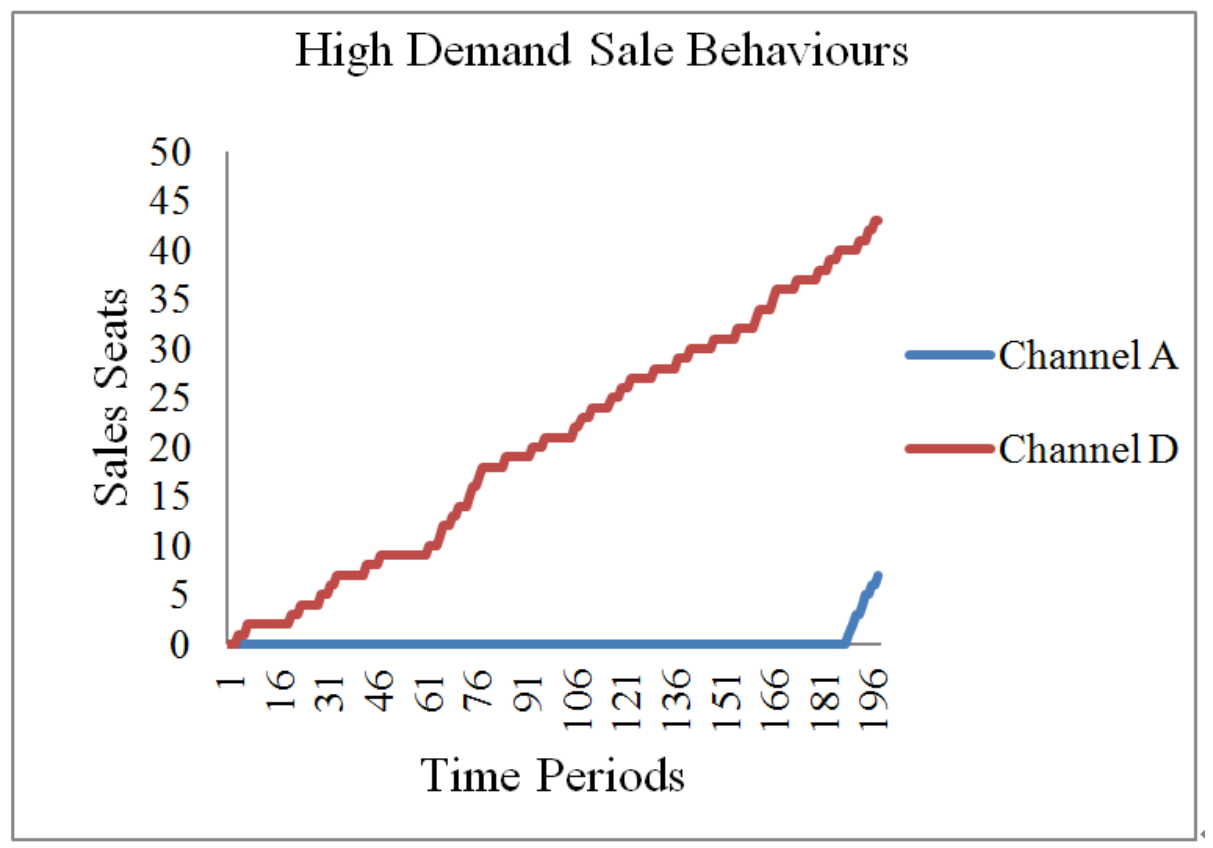

Figure 3.13: High Demand Pattern Sales Behaviours 
channel capacity deployment. After that, we have explored the optimal policy for the integrated model to maximum the airlines revenues and proposed several properties corresponding to the optimal policy. Furthermore, we proposed a customer shift behaviour model on the basis of the integrated model and discovered the optimal policy for that. The numerical experiments illustrate the different applications of the model and provide several implications for airline companies. Firstly, our system can keep the revenues more stable when channel commission rates increase. This implication decreases the lost revenue risk by contracting with the channel and holding the dominant position in airline market. Secondly, multiple indirect sales channels will not reduce airline company revenues, and, conversely, more indirect channels might bring more profits for the airline company. Therefore, the airlines should introduce more channels to improve their revenues. Thirdly, the demand forecast dominates a significant position in our system. The accurate prediction of demand improves the revenues for the airline company. After that, our system integrates the channel and fare class, and there is not an obvious conflict between the channel and fare class allocation. The airline company can realize channel control on the basis of the revenue system. However, we need to note the observation that the increased rate of the DCAM with multi-fare and without multi-fare brings a small difference. This might be due to the combined but complex effects of the fare class and the channel distribution. As discussed in Section 5.4, the assumption of customer arrival is one explanation for the result. A more thorough investigation is needed in a future study. Thus, our study solves a mathematical problem, and it has value for practical use. Finally, all applications we proposed have better performance in dealing with the problem of multi-fare and multi-channel capacity control than the classic independent system. The key contribution of this study is that it is the first attempt to integrate the capacity allocation decision and distribution channel decision in order to improve the revenue management. The modelling framework provides a basis for investigating relevant issues in future studies. The optimal policy together with the observations from numerical examples brings insights on the directions where revenues can be improved in practice. Thus, from both the theoretical and practical perspectives, this study contributes to the knowledge development in the field. 
4 DCAM Model with Channel-shift Customer Behaviour

While the chapter 3 deal with single-leg capacity allocation and channel distribution problem. In the DCAM model, we assumed that demand for each channel is independent. More specifically, when the airline makes a decision of one channel, they do not consider the other available channel. From the customer side, the control model assumes that if the request is rejected by one channel, the demand will lost. However, this hypothesis is somewhat inconsistent with reality. For example, When a customer is rejected by a low value channel, He has a great possibility to buy the ticket from a high value channel and it is called channel-shift customer behaviour. When the customer choose another flight, it is called lost sales. Obviously, this kind of customer behaviour often occurs in reality and needs to be considered in the control model.

\subsection{Traditional capacity allocation model with customer behaviour}

In the traditional capacity control model, similar behaviours of customers are also considered. In traditional model, it is called buy-up model. The buy up model relaxed one of the six assumptions we introduced in the previous section(See section 3.1.1), which is that the demand is independent for each fare class. The model considered that the customers would buy a higher fare class when they rejected by a low fare class. Of course, this kind of customer behaviour can also occur, but it is less likely to occur than the channel-shift customer behaviour. Needles to say, for a customer, if he buys a ticket at a higher fare class, he will increase their ticketing cost, but to buy from high value channel will not increase his cost. Therefore, it is more practical to consider channel-shift customer behaviour.

Next we will first introduce some traditional buy-up models in section 4.1.1 and 4.1.2. After that, we will define our channel-shift problem in section 4.2 and propose our model in section 4.3. Finally, we use some experiments to verify the channel-shift model in section 4.4.

\subsubsection{Traditional Static buy-up model}

In the previous research on the buy up model, the first step was to start with the traditional static revenue management model(See Belobaba(1987a,b,1989),Belobaba and Weatherford(1996),Weatherford et.al(1993), Bohutinsky(1990). For example, two class buy-up model is discussed based on the Littlewood's model we have introduced in section 3.1.1. To discuss the 
two class buy-up model, we review the Littlewood model here. When the customer for fare class 2 comes, he will be accepted only if the class 2 fare is greater than the marginal price, which is $f_{2} \geq f_{1} \operatorname{Pr}\left(D_{1} \geq x\right.$ ) (See equation (3.1)). Additionally when the class 2 fare is lower than the marginal price, which is $f_{2} \leq f_{1} \operatorname{Pr}\left(D_{1} \geq x\right)$, the customer will be rejected. However, to the buy-up model, it has a probability $q$ that the customer will buy class 1 instead of class 2. The optimal formulation is below:

$$
f_{2}-f_{1} \operatorname{Pr}\left(D_{1} \geq x\right) \geq q\left(f_{1}-f_{1} \operatorname{Pr}\left(D_{1} \geq x\right)\right)
$$

or equivalently

$$
f_{2} \geq(1-q) f_{1} \operatorname{Pr}\left(D_{1} \geq x\right)+q f_{1}
$$

Note that If the customer buy up to class 1 , the net benefit for airline is $f_{1}-f_{1} \operatorname{Pr}\left(D_{1} \geq x\right)$. The left side is the net revenue for airline if the airline accept the customer for class 2, which is the Littlewood's rule. The optimal balance is whether to open class 2 or close class 2 and let the customer have probability $q$ to buy up to class 1 . If the net benefit for airline in class 2 is higher than the expected net revenue for airline up to class 1 , the airline will still open class 2, otherwise close the class 2. The process is shown in figure 4.1.

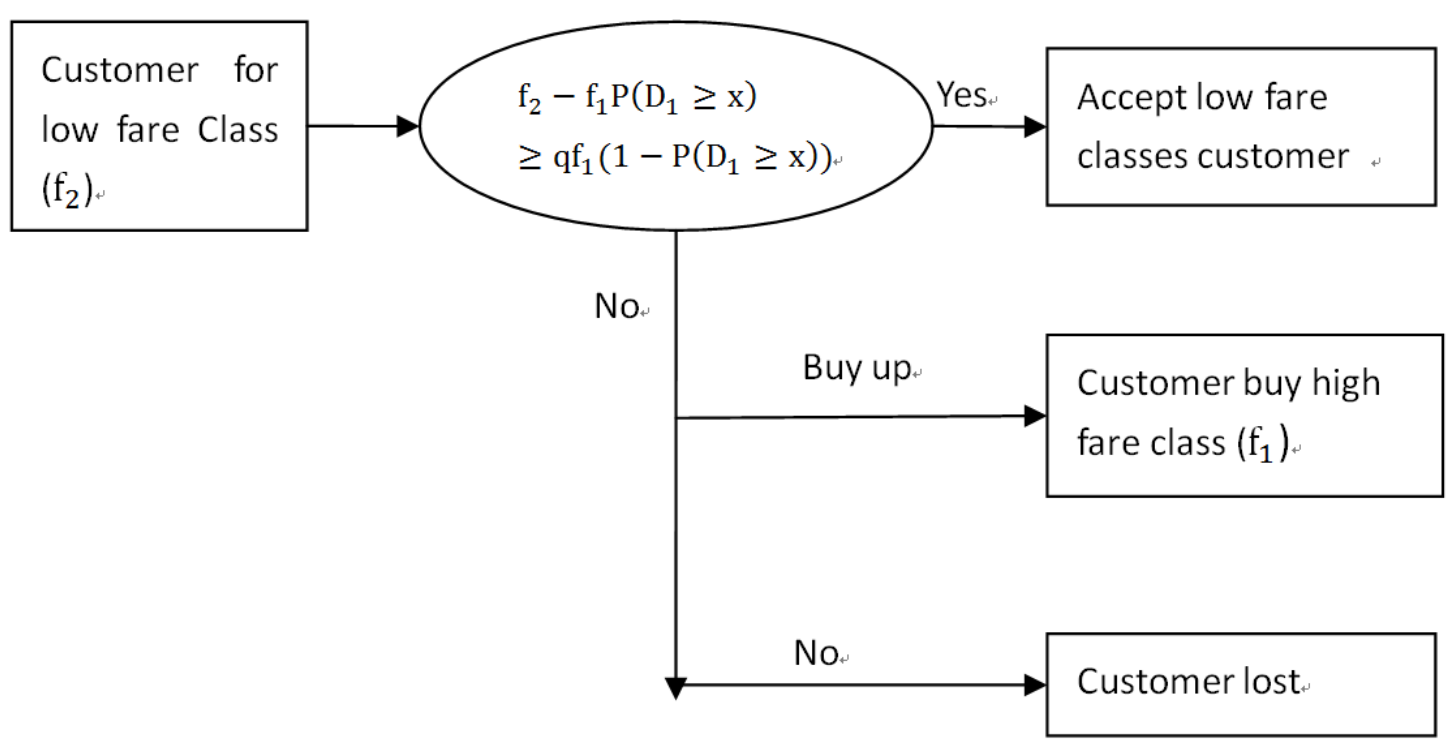

Figure 4.1: Static two-class buy up model booking process 
The development of the buy-up model relies on the development of traditional revenue management models. Like the traditional two-class model, the twoclass buy-up model can not solve the multi-class buy up problem in reality. For example, when one class is closed, the decision can not only depend on the upper class or the lower class. The buy up behaviour is also complex. Some one may buy two higher class or three higher class. Therefore, the multi-class model is not a simple binary model and become a complex multi-nominal model.

We have mentioned a heuristic method EMSR in traditional static single-leg, which dealing with the multi-class model(See section 3.1.1). Through introducing the buy up factor into EMSR heuristic model, the researcher try to solve the multi-class buy up problem. The equation is below:

$$
\tau_{j}=\sum_{i=1}^{j-1} P r_{i}^{-1}\left(q_{i} f_{i}-f_{j} /\left(1-q_{i}\right) f_{i}\right)
$$

Where $q_{i}$ is the probability that the customer of class $j$ buys up to class $i$, $i \in 1, \ldots, j-1 . f_{i}$ is the fare of class $i$ and $\tau_{j}$ is the protection level of class j. $\operatorname{Pr}_{i}$ is the demand distribution of class $i$. The EMSR buy up model (4.3) is developed from the original EMSR model (3.7).

Though the static buy up model has proved useful in practice(Bohutinsky,1990), it is undeniable that the model still has certain flaws as the traditional static model. The most of typical of these is the customer arrival order. In the next section, we will introduce the buy-up factor in dynamic model.

\subsubsection{Traditional dynamic buy-up model}

Dynamic fare-class buy-up Model was developed by You(2001). This model extended the static model, which we have mentioned above, to a dynamic multiclass model. The difference between dynamic model and static model will not be discussed again here(See Chapter 3). Here we introduce the dynamic buy-up Model.

As the traditional dynamic model in section 3.1.2, the objection of the value function is to maximize the expected revenue in a determined selling period. However, the dynamic buy-up model has one more decision compared with the traditional DP model. For example, to traditional DP model, it is assumed that when one demand comes, the airline has to decide whether to accept it or not. If the customer is rejected, this demand is lost. But to buy-up model, after the customer is rejected, the model assumed that the customer has some probability to buy up to a higher class. At this time, the airline should make 
another accept/deny decision for the return customer. The buy-up DP model is illustrated below:

$$
v_{t}(x)=v_{t-1}(x)+\sum_{i=1}^{n} \lambda_{t}^{i} \max \left\{g_{t}^{i}(x, c), r^{i}-\Delta v_{t-1}(x)\right\}
$$

Where

$$
g_{t}^{i}(x, c)=\sum_{l \in c} q_{l}^{i}\left(r_{l}-\Delta v_{t-1}(x)\right), i \geq 2
$$

Compared with the DP model, the decision of the buy-up model(equation 4.4) not only depends on the net revenue of the class $i$, which is $r^{i}-\Delta v_{t-1}(x)$, but also considers the expected net revenue of buy-up class $l$, which is the equation (4.5).

According to the development of the buy-up model, this model provides two important contributions to the study of the buy-up model. The first one is for the traditional DP model, which breaks an assumption that when a customer is rejected, the demand will be lost. The second one is for the static buy-up model, which releases an assumption of the strict low-high class order . Relaxing these two assumptions makes the buy-up model closer to the reality. The model of this paper is based on the inspiration of this model and is combined with the multi-channel model.

\subsubsection{Summary}

The buy-up-related models, whether static, heuristic, or dynamic, provide a solution that responds to customer choice behaviour. At the academic level, some experiments with these models have demonstrated that the buy up model has increased the revenue of airlines. At the same time, there can be some analysis and feedback on the customer's behaviour. Of course, the model itself also has some limitations and assumptions that require more researchers to release it to get closer to reality. In addition to the limitations of the model itself and some assumptions, the buy up model still has some difficulties at the implementation level. For example, how to estimate the buy-up factor is very difficult for airlines. In the model we mentioned above, they always simply assume a reasonable-sounding factor. However, sometimes there are some degrees of deviation from the actual situation. In addition, the buy up model easily duplicates customer demand forecasts. This will also have a certain influence on the capacity control. Despite some deficiencies, the buy-up model proved to be effective in practice. 
As we mentioned above, customer choice behaviour has a certain influence on capacity control. Due to the development of the Internet, in most cases, customers no longer face the airline directly, but face various channels. The customer choice behaviour in the face of the channel also has a great influence on the airline's channel strategy. In the next section we will propose solutions to the customer's choice of behaviour in the channel.

\subsection{Problem definition}

In this section, we analyse the customer channel shift behaviour and present a customer channel shift DCAMP model (DCAMS). We define customer shift behaviour as: when a customer is rejected by a high-cost channel, the customer has a probability to shift to a low-cost channel(Figure 4.2). As mentioned in Section 3.2.3, when demand occurs, there exists a set of channels which are capable of fulfilling the demand, i.e. with only $i_{j} \leq \hat{i}_{j}(t, x)$. Channel shift customer behaviour will more likely happen in reality than the fare buy-up behaviour (You, 2001,see section 4.1.2), because shift behaviour will not add more cost for customers. Therefore, channel shift behaviour will affect on the decision of channel capacity allocation.

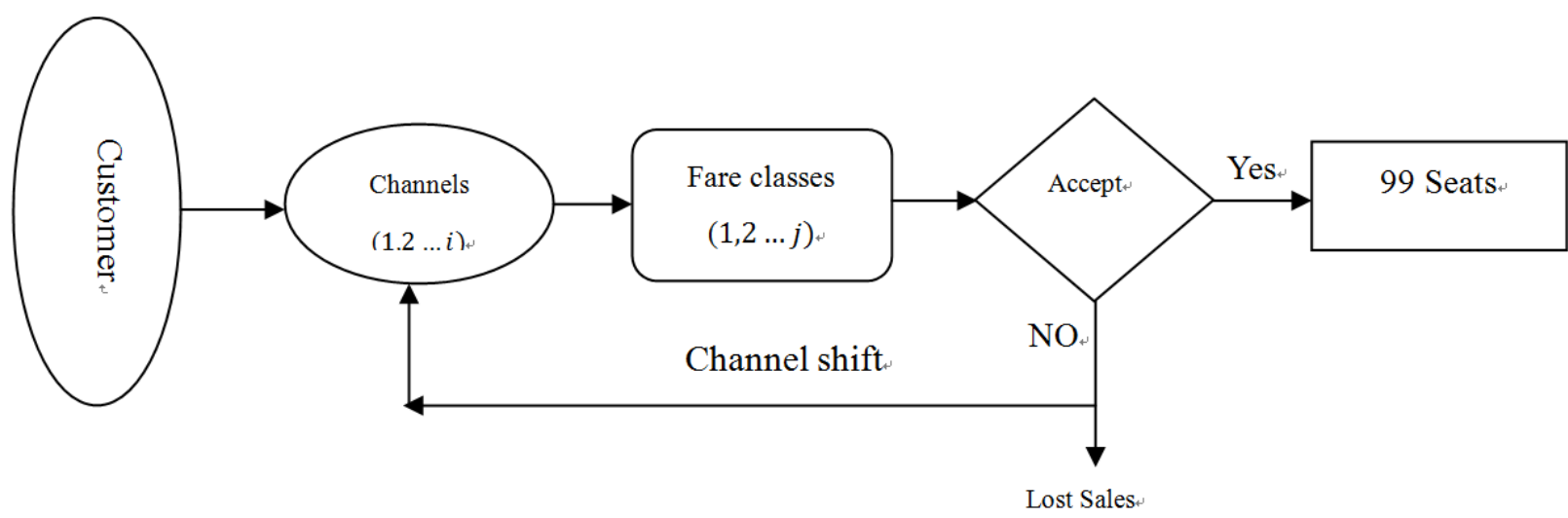

Figure 4.2: Channel Shift Decision Process

We formulate the DCAMS model basing on the DCAM model assumption (see Chapter 3). So there are some assumptions that are the same as the DCAM model. For example, we assumed that at most one customer arrives in each period. The arrival rate of each fare class $j$ and channel $i$ is $\lambda_{i j}$. It holds that $\lambda_{0}+\sum_{i=1}^{m} \sum_{j=1}^{n} \lambda_{i j}=1$, where $\lambda_{0}$ is the probability that no booking requests in that period. In addition to the original customer-based assumptions, we extend some assumptions about the channel-shift behaviour. We assume that if a customer is rejected by channel $i$ in time period $\mathrm{t}$, then the customer determines to buy the tickets through channel $l$ at the same period with a probability $\sigma_{l t}$. 
General conditions imposed on $\sigma_{l t}$ are $\sigma_{l t} \geq 0$ and $\sigma_{0 t}+\sum_{l=i}^{m} \sigma_{l t}=1$ where $\sigma_{0 t} \geq 0$ denotes the probability of having no further booking requests for the denied booking of channel $j$ at time $t$. If the customer is rejected for the second time, we assume that the customer is completely lost. We also define that the commission fee rate $\delta_{i}$ can be nested by $\delta_{0} \leq \delta_{1} \leq \delta_{2} \ldots \leq \delta_{m-1} \leq \delta_{m}$, where $i \leq l \in\{0, \ldots, m\}$. The condition denotes that the customer can only shift to low-cost channel.

The first assumption is a standard assumption for the DCAM model in chapter 3. The second and third assumption describe the possibility of channel-shift customer behaviour. Therefore, our channel-shift model make an improvements on the traditional RM models and multi-channel RM model which do not consider the channel-shift behaviour.

To the DCAMS problem, the airline has to make three decisions at each time period $t$ :

1. Accept or reject a booking request from Channel $i(\mathrm{D} 1)$.

2. Accept or reject a booking request for fare class $j(\mathrm{D} 2)$.

3. In the case of rejection in D1 and if the customer wants to shift to channel $l$, accept or reject the customer's new booking request(D3).

As DCAMS system has three decisions for channel allocation, we introduce the binary channel variables $w_{i} t$, the binary fare-class variables $u_{j t}$ and another binary channel-shift variable $\eta_{l t}$ to represent decisions D1, D2 and D3 for any time period $t$, channel $i$, fare-class $j$, and shift channel $l$. In particular, $u_{j t}=1$, $w_{i t}=1$ if and only if a booking request for fare class $j$ from channel $i$ is accepted. When $u_{j t}=1, w_{i t}=0, \eta_{l t}=1$ if and only if an upgrade to channel $l$ from $i$ is accepted, Which represents that if the channel $i$ rejected customer is accepted by channel $l$ at the time period $t, \eta_{l t}$ equals to 1 ; otherwise 0 .

The DCAMS problem can be formulated as a dynamic programming model. The model is formulates as the following section.

\subsection{Channel-shift Model}

Parameters:

$i: \in\{0, \ldots, m\}$ represents every channel in a single-leg flight.

$j: \in\{0, \ldots, n\}$ represents the every fare class in a single-leg flight.

$t$ : represents each time period.

$T$ : finite horizon periods.

$N$ : totally identical products in one leg flight

$w_{i t}$ : the channel decision binary indicator such that $w_{i} t=1$ if the airline company accepts channel $i$ 's request in time period $t$, and 0 otherwise. 
$u_{j t}$ : the fare class decision binary indicator such that $u_{j t}=1$ if the airline company accepts fare class $j$ request in time period $t$, and 0 otherwise.

$\delta_{i}$ : channel $i$ 's commission fee rate.

$R_{j}(t)$ : revenues made from fare class $j$ in time period $t$.

$x$ : the integer variable representing the number of products left (capacity).

$V_{t}(x)$ : the maximum expected revenues of the remaining capacity $x$ in period $t$.

Additional Parameters:

$\eta_{l} t$ : the channel binary indicator such that $\eta_{l t}=1$ if the channel $i$ rejected customer is accepted by channel $l$ at the time period $t$, otherwise 0 .

$\sigma_{l t}$ : the probability representing the customer buy the tickets through channel $l$ in the period $t$ after the customer rejected by channel $i$.

The DCAMS model can be formulated as follows:

$$
\begin{aligned}
V_{t}(x)= & E\left[\operatorname { m a x } _ { w _ { i t } \in ( 0 , 1 ) , u _ { j t } \in ( 0 , 1 ) , \eta _ { l t } \in ( 0 , 1 ) } \left\{w_{i t}\left(1-\delta_{i}\right) u_{j t} R(t)+V_{t+1}\left(x-w_{i t} u_{j t}\right)+\right.\right. \\
& \left.\left.\left(1-w_{i t}\right)\left[\eta_{l t}\left(1-\delta_{l}\right) u_{j t} R(t)+V_{t+1}\left(x-\eta_{l t} u_{j t}\right)\right]\right\}\right]
\end{aligned}
$$

where the constrains for the DCAMS model is :

$$
w_{i t}+\eta_{l t} \leq 1, w_{i t} \in\{0,1\}, \eta_{l t} \in\{0,1\}
$$

This constrain limits that at one period only one customer can come, and only when this customer are rejected by the $i$ channel, there will be some probability that the customer can ask for $l$ channel.

where there are also two boundary conditions as the DCAM model (3.11):

$$
\begin{aligned}
& V_{T}(x)=0, \forall 0 \leq x \leq N \\
& V_{t}(0)=0, \forall 0 \leq t \leq T
\end{aligned}
$$

The same as the DCAM boundary condition, the boundary condition 4.7 represents that no matter how many capacity left, the expected value will be zero at the end of time periods. Additionally, the boundary condition (4.8) sets the capacity boundary, which means that when all capacity are sold out, the expected value will also be zero. 
For computing and analysing, the model (4.6) can be written as the following equation:

$$
\begin{aligned}
V_{t}(x)=V_{t+1}(x)+ & \sum_{i=1}^{m} \sum_{j=1}^{n} \lambda_{i j}(t) \max \left\{\left(1-\delta_{i}\right) p_{j}-\Delta V_{i t}(x),\right. \\
& \left.\sum_{l=i}^{m} \sigma_{l t} \max \left[\left(1-\delta_{l}\right) p_{j}-\Delta V_{l t}(x), 0\right], 0\right\}
\end{aligned}
$$

The optimal policy for DCAMS model is also based on the DCAM model. We also use bid price policy to optimize the model.

The optimal policy for DCAMS model can be simplicity explained as follows. First, we define $\Delta W_{i t}=\sum_{l=i}^{m} \sigma_{l t} \max \left[\left(1-\delta_{l}\right) p_{j}-\Delta V_{l t}(x), 0\right]$. Therefore, $\Delta_{W}$ it is a non-negative variable and represents the net revenue after rejecting the first choice channel $i$. That implies that when the rejected customer is accepted, the revenue of fare class $j$ from channel $l$ must larger than the bid price of channel $l$, where $\left(1-\delta_{l}\right) p_{j} \geq \Delta V_{l t}(x)$. The policy of deciding the first channel choice is also related to the $\Delta W_{i t}$. If the first booking request is accepted, the net revenue of accepting the request must be larger than the net revenue of rejecting it, where $\left(1-\delta_{i}\right) p_{j}-\Delta V_{i t}(x) \geq \Delta W_{i t}(x)$.

Compared the DCAM model or the traditional DP model, the revenue of the fare class $j$ from channel $j$, where $\left(1-\delta_{i}\right) p_{j}$, is not only larger than the bid price, but it should larger than bid price minus the net revenue of rejecting the first request $\Delta V_{i t}(x)+\Delta W_{i t}$. Therefore, there will be a new bid price for the customer shift model:

$$
\pi(x)=\Delta V_{i t}(x)+\Delta W_{i t}
$$

We point out that the DCAMS (4.6) reduces to that presented in You(2001) in a single-leg setting without channel factor(See section 4.1.2). And also the DCAMS model reduces to the DCAM formulation without channel-shift factor presented in Chapter 3.

\subsection{Numerical experiments}

\subsubsection{Design of experiments}

In this section, we design several experiments for demonstrating the properties of the channel customer shift model. Hence, there will be amount of problems 
Table 4.1: Experiment list

\begin{tabular}{c|cccc}
\hline Experiments & No.of Channels & Demand pattern & Commission Fee Case & Shift rate case \\
\hline 1 & 2 & 1 & 1 & 3 \\
2 & 2 & 1 & 4 & 1 \\
3 & 2 & 3 & 1 & 1 \\
\hline
\end{tabular}

to be investigated. We have illustrated some representative examples (Table 4.1) to demonstrate the characteristics of our model and the outcomes when decisions are integrated.

The first experiment was used to test the impact of our model on the revenue system in the three customer transfer rates. Among these, we set the same demand and the same agency rates for the two channels.In the second experiment, we considered the impact of different agency fees on the customer transfer behaviour model. Here we set the same shift rate, the same demand and four commission fee rate for the two channels. In the third experiment, we verified the application of the model under different requirements. We set the same commission fee rate, the same shift rate and three different demand patterns for two channels.

\subsubsection{Channel-shift in different shift rate}

The first experiment presents the effect of customer shift behaviour on channel allocation based on the model DCAMS and testify the effect of shift rate on airline's revenue by implementing our policy. In order to more accurately represent the impact of shift rate on the revenue system, we use the most basic channel model. There are two channels and one fare class. The specific parameters can be seen in Table 4.2 .

Table 4.2: Channel Characteristics for Experiment 4.4.2

\begin{tabular}{c|c|c|c}
\hline Capacity & 50 & Time periods & 100 \\
\hline Channel & Market Share & Fare Class(Fare) & Commission Rate \\
\hline A(CH.A) & $74 \%$ & 1 class $(260)$ & $13 \%$ \\
D(CH.D) & $26 \%$ & 1 class $(260)$ & $0 \%$ \\
\hline
\end{tabular}


In this experiment, the flight leg has 50 capacities and the time period for selling is $T=100$. Through 1000 runs of the simulator for different shift rates, the results are shown in Table 4.3. The experiment compares the revenues of DP model, DCAM model and DCAMS model, the seat allocation into different channel(Channel A and Channel D) and the increase rate in different shift rates. Table 4.3 illustrates that with the increase of customer shift rate, DCAMS makes more revenue improvements than DCAM without considering customer shift behaviour. Although when the shift rate is $11 \%$ the improvements from DCAM to DCAMS is very low and it does not seem to improve, when the customer shift rate increase to $31 \%$ the new system make more improvements. Through the tracking of the seat allocation, we can see that more customers use the direct channel( Channel D) to purchase seats as the shift rate increases. This phenomenon also confirms that as the shift rate increases, the airlineâĂŹs revenue is also increasing. This result implies that airlines can depend on promoting the customer shift behaviour to increase their revenues

Table 4.3: Results of Experiment 4.4.2

\begin{tabular}{c|cccccc}
\hline Rev for DP & \multicolumn{2}{|c|}{11749.4} & Rev.DCAM & \multicolumn{2}{c}{12218.5} \\
\hline Shilt Rate & Rev.for DCAMS & Seat allocation CH.A & Seat allocation CH.D & Inc.rate from DP to DCAMS & Inc.rate from DCAM to DCAMs \\
\hline 0.11 & 12269.8 & 0.4321 & 0.5679 & $4.24 \%$ & $0.4 \%$ \\
0.21 & 12363.2 & 0.3768 & 0.6232 & $4.96 \%$ & $1.23 \%$ \\
0.31 & 12452.8 & 0.3238 & 0.6762 & $5.64 \%$ & $1.92 \%$ \\
\hline
\end{tabular}

In addition, we analysis the sales through the sales behaviour figure 4.3, 4.4 and 4.5. According to the three figures, we can see that direct channel can make more improvements with the increasing shift rate. For airline direct sales channels, its lack of customers led to them selling fewer tickets.

As we can see in Figure 4.5, the customers' channel-shift behaviour in the previous and mid-term plays a significant role, resulting in a certain of customers transferring from high volume indirect channel to direct channel. And thus the behaviour increases the airline's revenue. The reason is that due to the DCAM's optimization strategy, the rejection rate of indirect channel in the early and middle period is relatively high, so it will also lose a large number of customers. The optimization strategy of DCAMS exactly compensates for this shortcoming. In the final stage, indirect channels make up for the shortcomings of insufficient direct channels, and the acceptance rate is relatively high. Therefore, the impact of DCAMS strategy in the later stage is relatively small. 


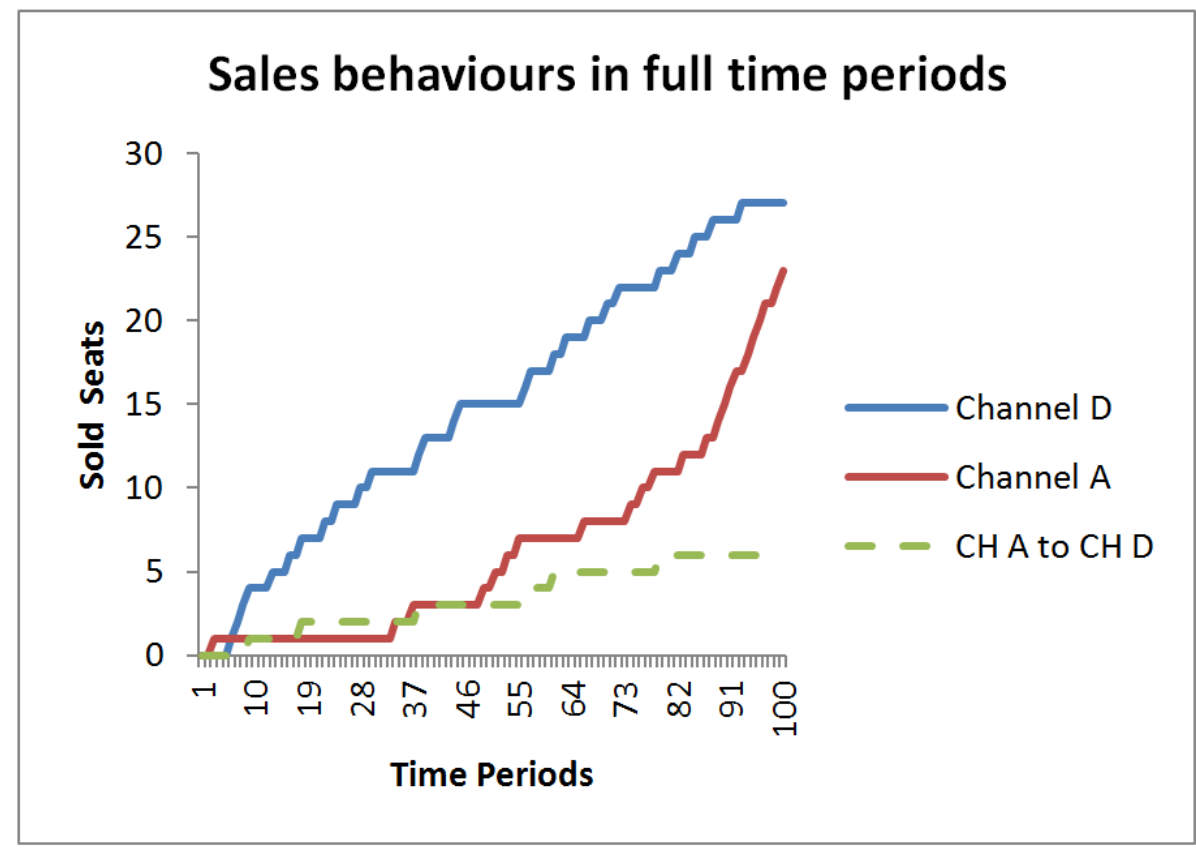

Figure 4.3: Low Shift Rate Sales behaviour for DCAMS

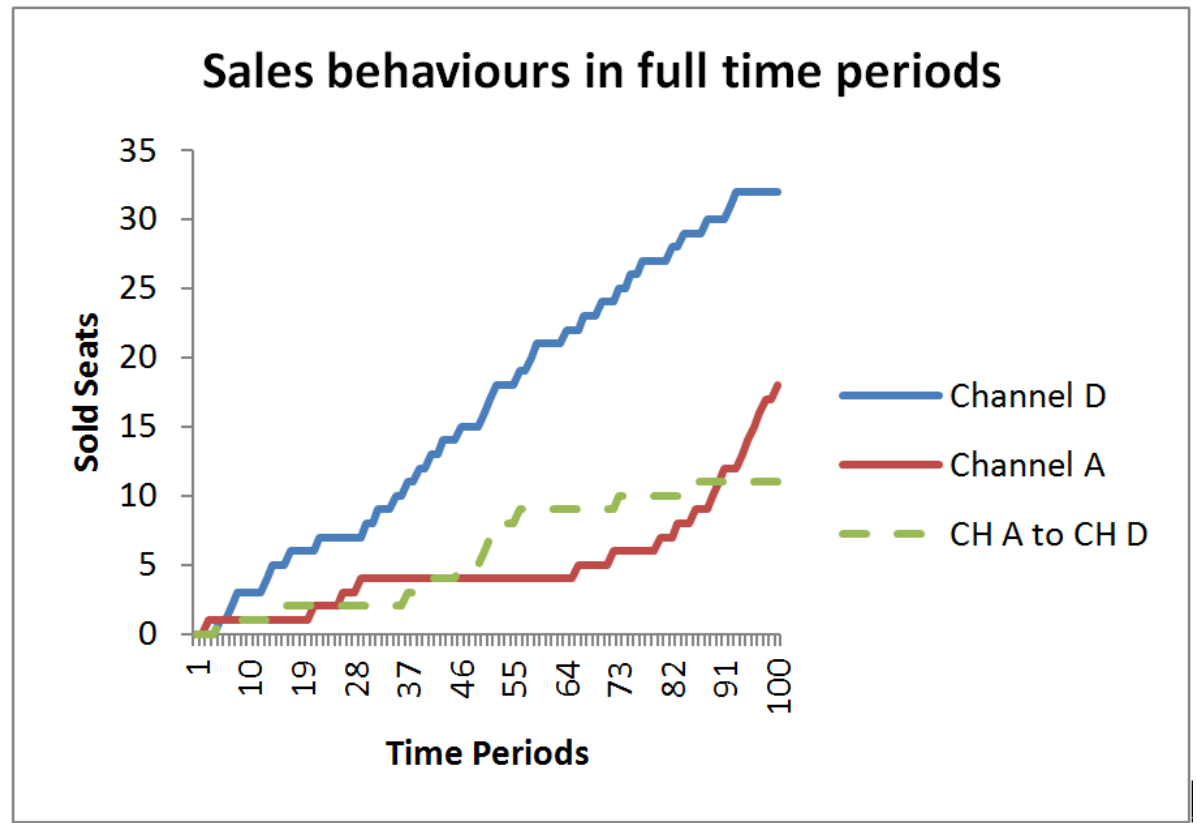

Figure 4.4: Middle Shift Rate Sales behaviour for DCAMS 
For example, as the experiment of shift rate $31 \%$, the acceptance rate in first 50 periods is $40 \%$. For the DCAM model, the acceptance rate in first 50 periods is only $28 \%$. The improvement of acceptance rate in the pre-term have an advantage for competing with other airline company.

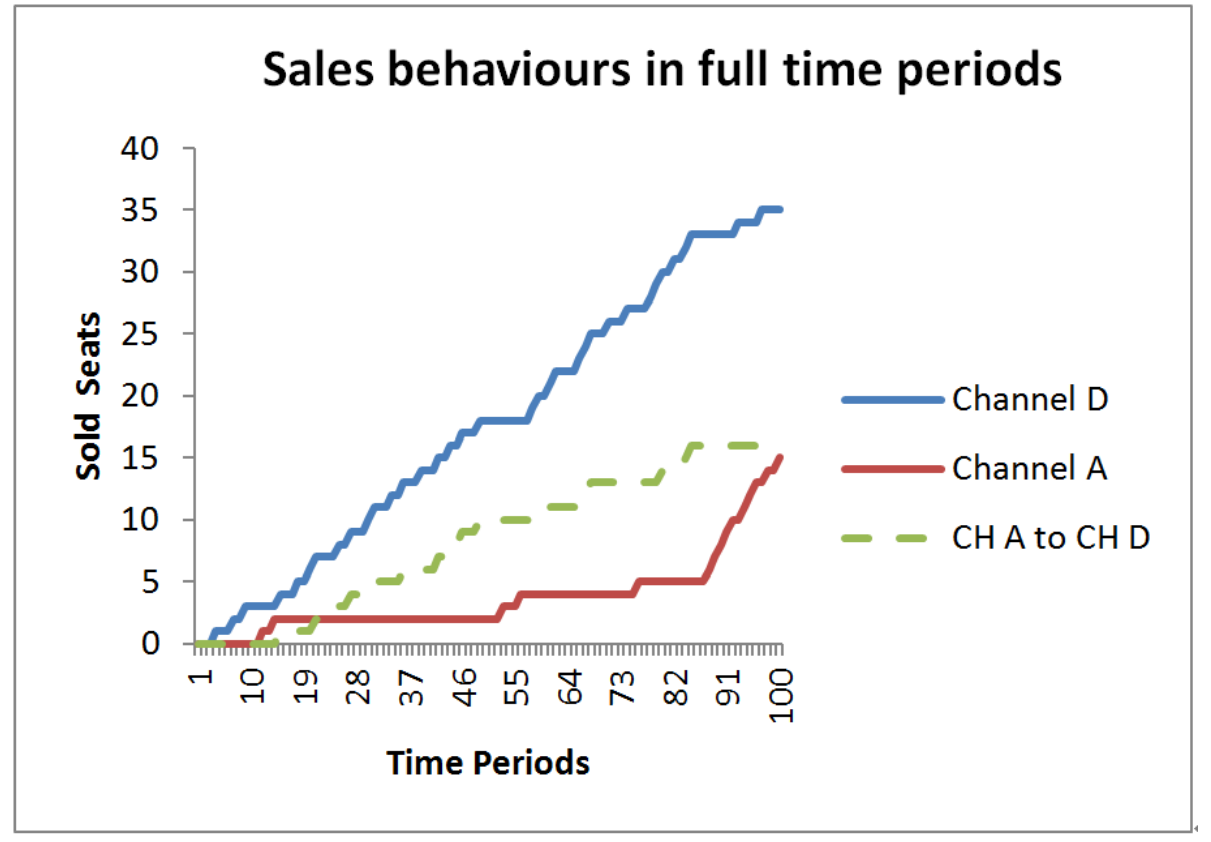

Figure 4.5: High Shift Rate Sales behaviour for DCAMS

In conclusion, Increasing the shift rate will increase the airline's revenue. From the perspective of sales behaviour analysis, the application of DCAMS strategy will increase the airline's pre-interim acceptance rate to reduce the loss of customers.

\subsubsection{Channel-shift in different commission rate}

In this experiment, We discuss the effect of the commission fee rate on the customer channel-shift model when the shift rate is constant. Here we also adopt double channel and one fare class example. The selling time period is $T=100$ and the total capacity is also 50. The shift rate of the example keeps in $31 \%$. The commission rate for the indirect channel(Channel A) is $13 \%, 23 \%$, $33 \%$ and $43 \%$ and the commission rate for the direct channel(Channel D) keeps in $0 \%$. The experiment is simulated 1,000 times with the random demand, and we assesses the average results.

As shown in figure 4.6, With the increase of commission rate, the channel model that considers customer channel-shift behaviour is more profitable than the common channel model.Therefore, when there are agents with relatively high agency rates, the airlines adopt a model that considers the customer's 


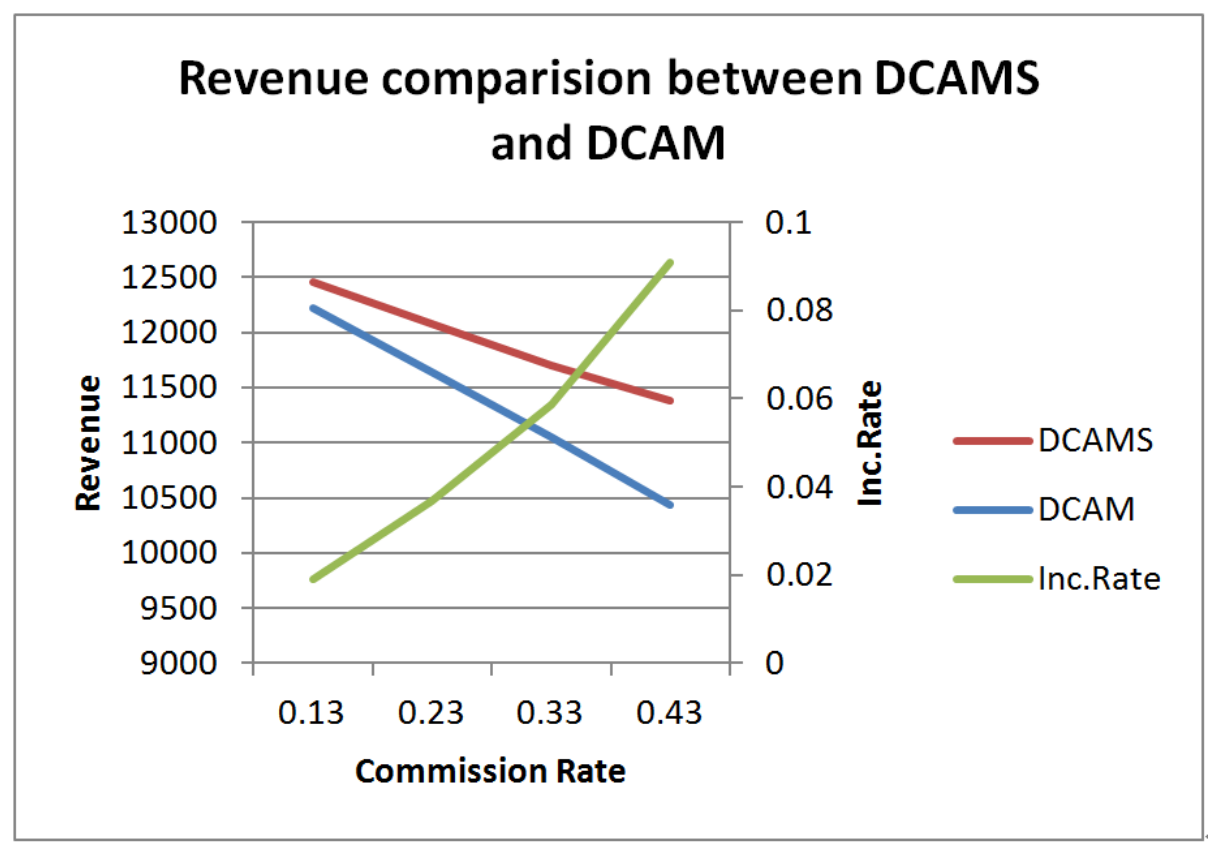

Figure 4.6: Revenue comparison over different commission rate

shift behaviour to bring excess profits to the company. Also considering the customer's shift behaviour, the airline can reduce the loss due to the increase of agency costs through this model.

\subsubsection{Channel-shift in different demand pattern}

The experiment is to discuss the impact of customer transfer behaviour on the airline's revenue under different demand conditions. As the basic assumption of DP model, the customer arrival rate for each time is 0.99 , which represents that there is a $99 \%$ possibility that one customer will come in each time period. Thus, the time period can be roughly viewed as the value of total demand. This experiment adopts the basic channel parameters of experiment 4.4.2. The commission rate is $13 \%$ and the shift rate is $21 \%$. The total capacity for each example is 50, and the time period is $30,100,200$, which represents the low demand, middle demand and high demand volumes, respectively.

According to the table 4.4, We can see that in the high demand and low demand, the model that considers the customer transfer rate gains almost the same as the model that does not consider the customer transfer behaviour. For the medium demand, that is, the demand matching with the accommodation, the profit obtained from the model that considers the customer's transfer behaviour is obviously higher than the model that does not consider the customer's transfer behaviour. 
Table 4.4: Results of Experiment 4.4.4

\begin{tabular}{c|ccccc}
\hline Time & Seat allocation of CH.A & Seat allocation of CH. D & Revenues for DCAM & Revenues for DCAMS & Inc.rate \\
\hline 30 & 0.4597 & 0.1403 & 7033 & 7023.11 & $-0.14 \%$ \\
100 & 0.3763 & 0.6237 & 12218.5 & 12364.1 & $1.18 \%$ \\
200 & 0 & 1.00 & 12958.3 & 13000 & $0.32 \%$ \\
\hline
\end{tabular}

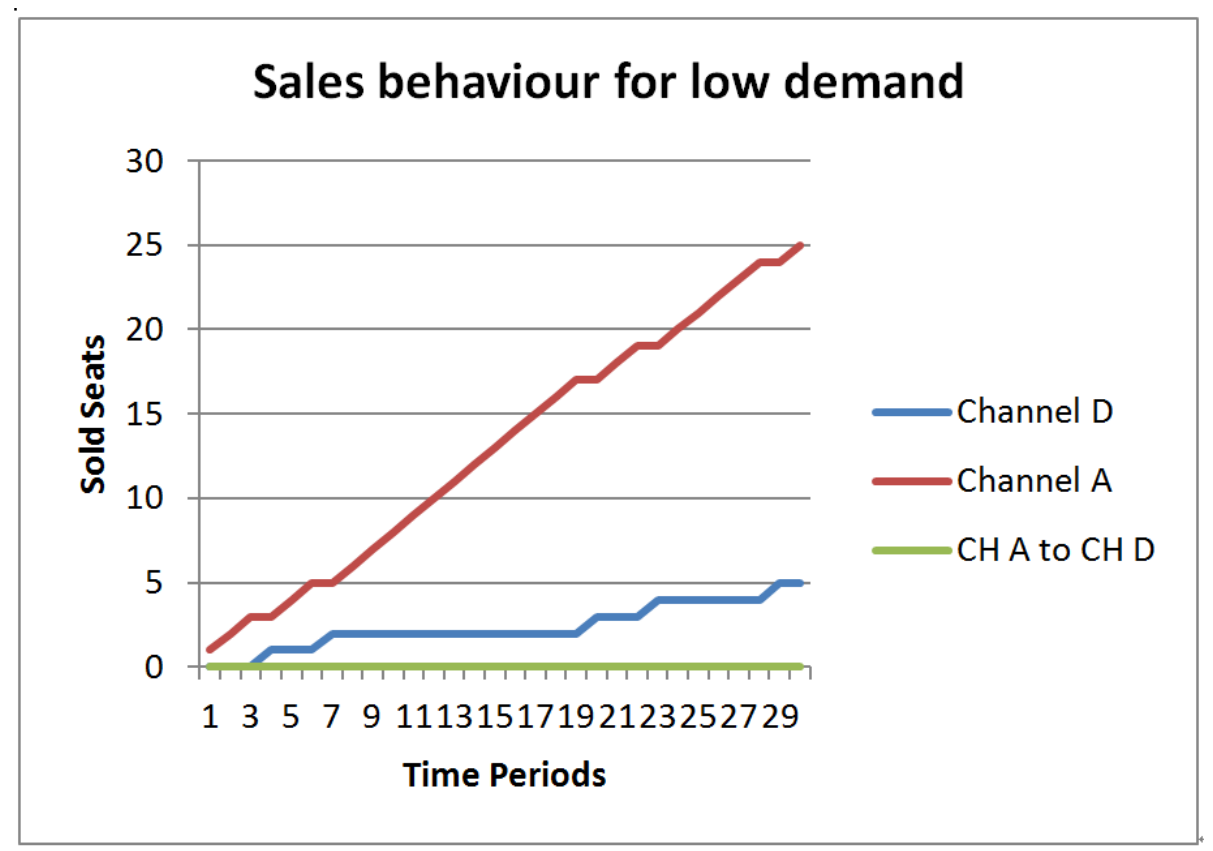

Figure 4.7: Sales behaviour for low demand in DCAMS 
Although the two models have the same benefits under low demand and high demand, the specific reasons are different. We clarify their reasons by analysing their sales behaviour. From the low-demand sales behaviour figure(see figure 4.7 , it can be seen that when the demand is not high, almost all the arriving customers are selected for acceptance during the sales process. Therefore, there is no customer transfer behaviour because there are no rejected customers. Because of this, the model that considers customer transfer behaviour is the same as the model that does not consider customer transfer behaviour. From the lowdemand sales behaviour diagram, it can be seen that when the demand is not high, almost all the arriving customers are selected for acceptance during the sales process. Therefore, there is no customer transfer behaviour because there are no rejected customers. Because of this, the model that considers customer transfer behaviour is the same as the model that does not consider customer transfer behaviour.

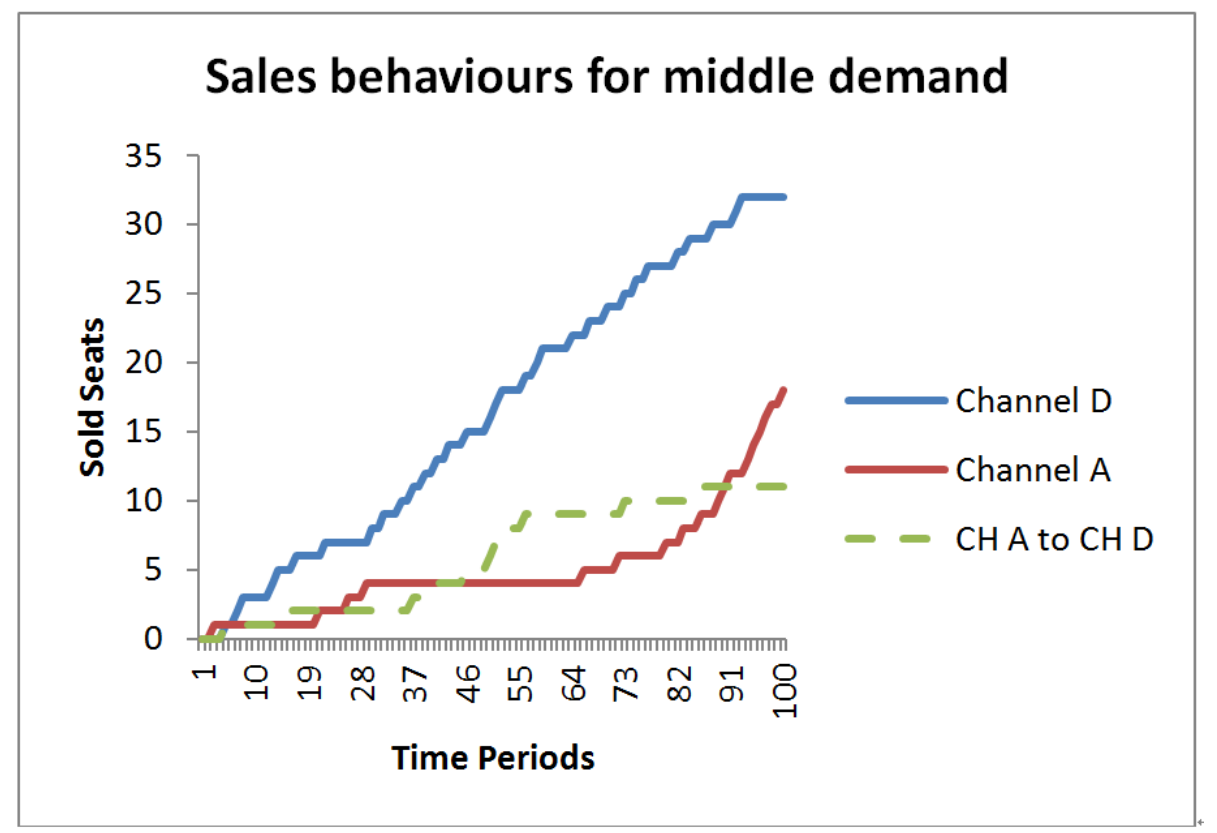

Figure 4.8: Sales behaviour for middle demand in DCAMS

In the case of high demand. the reason is different from the case of low demand. We are still seeking reasons from the high demand sales behaviour figure(See figure 4.9). As can be seen from the figure, due to sufficient demand, airlines have almost rejected all indirect channels in order to maximize the airlineâĂŹs revenue. This is why the model that considers the customer's transfer behaviour and the model that does not consider the customer's transfer behaviour have the same benefit. Because it can be seen from the figure 4.9 and 3.13 that both scenarios have almost reached the maximum benefit of the airline. 


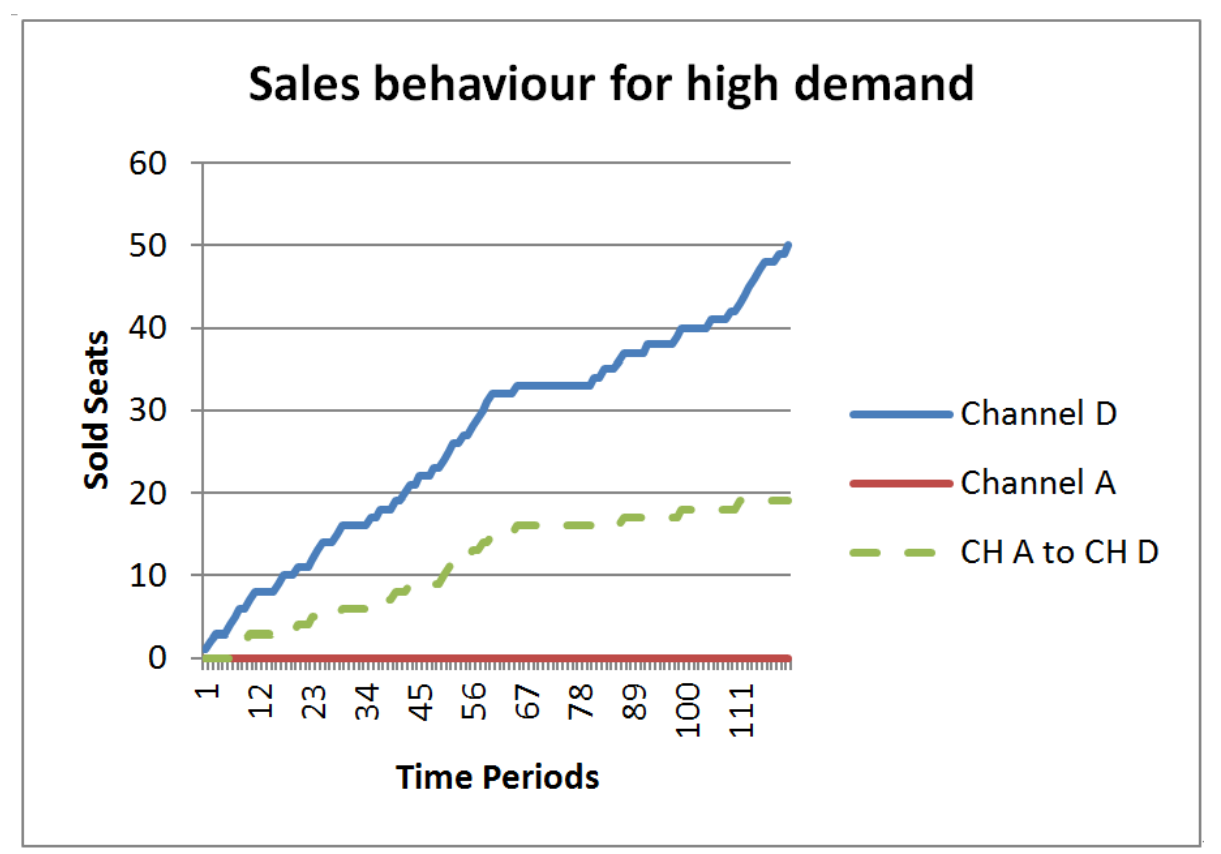

Figure 4.9: Sales behaviour for high demand in DCAMS

But in addition to the benefits we can see, the model that considers the customer's transfer behaviour produces some differences in the sales process compared to the model that does not consider the customer's transfer behaviour. In both cases, we set the time to 200 time periods. We can see that in the model that considers the customer's transfer behaviour, the airline has completed the sale of 50 capacities in only about 120 time periods. Under the same condition, which does not consider the customer's transfer behaviour, the airline needs to sell 50 seats in around 200 time periods. This phenomenon is well explained by our model and the actual situation. Because the model takes into account the customer's transfer behaviour, the demand for indirect channels that were rejected in the previous period has shifted to direct channels. In addition to greatly increasing the airline's acceptance rate for demand, it also reduces overall sales time. This has several important implications for the airline. First, as can be seen from this case, the rejection rate of models that do not consider customer transfer behaviour is too high. This is inconsistent with the reality and may have an adverse effect on the airline's demand forecast. Second, due to changes and increases in actual demand, it may have an impact on the airline's sales time and the schedule of the aircraft. For example, the last case airline can reduce sales time or change larger aircraft to increase revenue. Finally, airline demand forecasts have a certain influence on this model. 


\subsection{Conclusion}

In this chapter, We studied a multi-channel single-leg capacity allocation model that considers customer channel-shift behaviour. We propose a model that introduces customer transfer behaviour into multi-channel capacity allocation. After that, we have developed the optimal policy on the base of multi-channel capacity allocation optimal policy. The numerical experiments enumerate the impact of the model on the revenue management system under different circumstances and suggest various inspirations to the airlines. Firstly, Our system revenue will increase as customer transfer rates increase. This has a great effect on the revenue system considering multi-channel issues. When the customer discovers that a channel cannot purchase a ticket and transfer to other channels without any cost, there is a high probability that they will choose to purchase tickets through other channels. For airlines, it makes sense to make forecasts and research on the demand of transfer customer. Secondly, the model increases the acceptance rate of customers to reduce customer loss caused by the introduction of multi-channel factor. Retaining more customers means greatly improving the competitiveness of airlines. Thirdly, The model that considers customer transfer behaviour has better stability than the model that does not consider customer transfer behaviour. When agency fees increase, airlines can better reduce cost increases. Fourthly, The customer's transfer behaviour has a great influence on the demand forecast. Airlines can reduce sales costs by changing sales time through changes in demand. Or increase airline revenue by replacing larger aircraft. Finally, our model not only has better results than the traditional revenue management model, but also has better results than the multi-channel model. Our model is also more realistic than other models. 
5 Network capacity allocation with Multi-Channel distribution

\subsection{Traditional network model in revenue management}

At first, we will introduce the network revenue management in airline industry. In modern civil aviation service industry, the airline company usually sells a flight ticket with a set of flight legs. This kind of product is called an origindestination itinerary fare class combination by civil aviation service company. The basic network problem can be showed as figure 1. Airline can sell different leg combination products. For example, the airlines can sell a direct ticket from Shanghai(PVG) to Frankfurt(FRA). The airlines also can sell a transfer ticket which from Shanghai to Frankfurt and transit in Beijing(PEK). It is usually cheaper to take transit flight than the direct flight. Why are transit flights cheaper and how do they allocate the capacity and pricing? All this are pointing to network revenue management, what we will talk about.

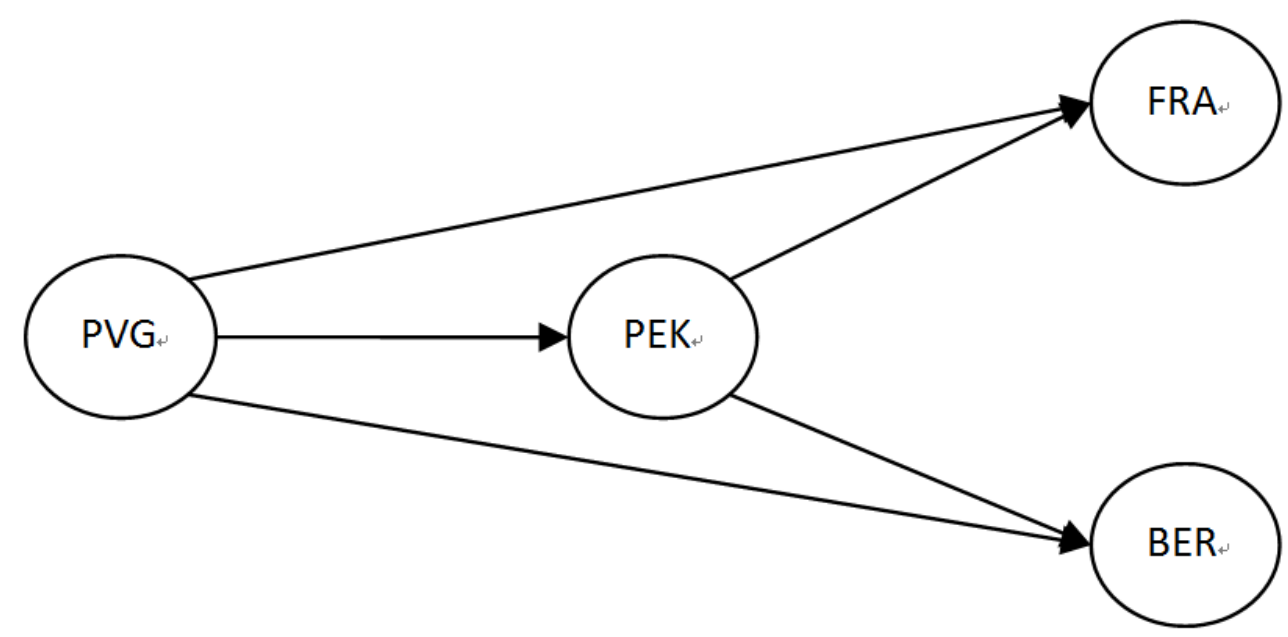

Figure 5.1: A simple airline network

When products are sold as legs combination, managing the capacities of each leg in a network will influence the airlines' revenue and operation. Therefore, it is important to integrate the capacity allocation on each resource for maximizing total revenues. 


\subsubsection{Dynamic network model}

This part will introduce the dynamic network model which is the most realistic and relevant model for network revenue management. The dynamic network model(DNM) can be found in Talluri and van Ryzin(2004, Sec 3.2). DNM model assumes a process of airline's selling tickets that when a request for one product comes, the airline makes a decision whether to accept or not. The model assumptions and model formulation is described below:

$$
r_{t}(x)=E\left[\max _{\omega \in(0,1)}\left\{\operatorname{Prod}(t) \omega+r_{t+1}(x-M \omega)\right\}\right]
$$

With boundary conditions:

$$
r_{T}(x)=0 \forall x
$$

$$
r_{t}(0)=0 \forall t
$$

It is assumed that there are $n$ products and $m$ legs in the network. Each product consists of several legs or one leg with a given price. The vector $\operatorname{Prod}(t)$ is the revenue of the product when the demand comes at the $t$ periods. There are $t=\{0, \ldots, T\}$ time periods in a finite selling horizon with the starting time 0 and $T$ as the time of departure. Each time period is assumed to be small enough so that only one arrival request in one time period. The state of the network is described by each leg remaining capacity vector $x=\left(x_{1}, \ldots, x_{m}\right)$. The initial capacity vector $s$ is $s=\left(s_{1}, \ldots, . s_{2}\right)$. Define the incidence matrix $M=\left[m_{i j}\right]$ where $m_{i j}=1$ if leg $i$, where $i=\{1, \ldots, m\}$, is used by product $j$, $m_{i j}=0$ otherwise. The vector $\omega=\left(\omega_{1}, \ldots, \omega_{n}\right)$ is the decision binary variable. When the arrival request from product $j$, where $j \in\{1, \ldots, n\}$, is accepted, the decision variable $\omega_{j}$ is coded to one. On the other hand, When the arrival request from product $j$ is rejected, the decision variable $\omega_{j}$ is coded to zero. The decision depends on the remaining capacity, the revenue of the product and capacity incidence in each flight leg. The objective of each decision in each time period is to maximize the total expected revenue. The boundary condition (5.2) represents that when the time ends, no matter how many capacities left, the expected revenue of $T$ periods will be zero. The boundary condition (5.3) indicates that the expected revenue of starting period is also zero.

Although DNM model is very close to practical situation, solving the network dynamic programming optimally is almost impossible because of the curse 
of dimensionality. For example, an airline network has 30 flight legs and each flight 150 seats. The state of airline network will be $150^{3} 0$. Therefore, the problem has to rely on the approximation methods. Generally there are two types of approximation methods. One is to use static mathematical programming to simplify the network model, like determined linear programming model, random linear programming model. Another type of methods is to decompose the dynamic network model to single-leg model, like approximation dynamic programming model. Next part we will introduce some approximation methods for solving the DNM mode

\subsubsection{Approximations based on dynamic network model}

This part will introduce several approximation methods. In network revenue problem, the objective of using approximation is to estimate the value function 13.1. One of the most widely used approximation is determined linear programming(DLP), which is first developed by Williamson(1992). DLP model assumes that the demand is given by the historical data or forecast. The basic DLP model is showing blow:

$$
\begin{aligned}
& r_{t}^{D L P}(x)=\max \sum_{j=1}^{n} \operatorname{Prod}_{j} b_{j} \\
& \text { s.t. } \sum_{j=1}^{n} \sum_{i=1}^{m} M_{i j} b_{j} \leq x_{i} \\
& b_{j} \leq M D_{j} \\
& b_{j} \geq 0
\end{aligned}
$$

The decision variables $b_{j}$ stands for the capacity allocation for each of the $n$ products. The demand is given by the mean demand $M D_{j}$. The objective of function (5.4) is to maximum revenue of each product times their capacity allocation. The constrain (5.5) states that the capacity allocation for each product must not exceed the capacity limit of each leg. The second constrain (5.6) indicates that the capacity allocation must not exceed the mean demand. The last constrain shows that the booking request must be non-negative. 
The advantages of DLP model is simple and easy to implement. However, DLP model utilizes determined demand as the system input, ignoring the demand uncertainty. Therefore some model begin to consider the demand uncertainty, for example, a randomized linear programming was developed by Talluri and Van Ryzin(1999). Next part we will introduce randomized linear programming.

The difference between randomized linear programming(RLP) and determined linear programming is demand input. RLP approximation incorporates the random demand $R D$ information replacing the determined demand $M D_{j}$ in constrain (5.6). The approximation linear programming to the value function is below:

$$
\begin{aligned}
& r_{t}^{R L P}(x)=\max \sum_{j=1}^{n} \operatorname{Prod}_{j} b_{j} \\
& \text { s.t. } \sum_{j=1}^{n} \sum_{i=1}^{m} M_{i j} b_{j} \leq x_{i} \\
& b_{j} \leq R D_{j} \\
& b_{j} \geq 0
\end{aligned}
$$

Although the RLP model seems like DLP model, RLP model provide a solution to consider the demand uncertainty. And also because of the similarity of DLP model, RLP model can be easily applied into traditional revenue systems. The RLP also was proved that the result is better than the DLP model in some test bed. The paper also mentioned there are some variance reduction techniques to improve the efficiency of the system in the future studies.

\subsubsection{Summary}

After several introduction of network revenue management model, we know that network management can be used in some service companies which sell products consisting of a combination of a bundle of resources and fare class. For instance, airlines those offer multi-leg services, hotel renting rooms for multi-nights, and 
car rental for multi-days. Network revenue management is important for these perishable products service company. Comparing the single-leg revenue management, computation complexity and the scale of network is the key challenge in network. As we mentioned in section 5.1.1, general dynamic programming is hard to be solved because of curse of dimensionality. Therefore, some approximations are developed for solving the large scale of network problem.

As mentioned above, the computation of the traditional network revenue management has already been very complex. How to integrate the channel factor problem into the revenue management problem and solve the computational complexity problem has become the key role to solve the problem of the channel network. We first propose a dynamic revenue management with multi-channel model based on the revenue management network model. At the same time, due to the computational complexity of the dynamic model, we propose a deterministic linear programming model to solve the original problem. Therefore, we have simplified complex mathematical problems into solutions that can be applied in the industrial field.

In the next section, we will introduce our new network model and approximation method. In the end, we will use the experiments to illustrate the results and give some implications.

5.2 Network capacity allocation with Multi-Channel distribution

\subsubsection{Problem Definition and model}

Assume the airline company selling $\mathrm{n}$ products(itineraries) in a $\mathrm{m}$ resources(legs) network. A product is a combination of a bundle of resources and fare class. Each product $j$ has its price $p_{j}$, where $j \in\{1, \ldots, n\}$. The products are sold by $h \in\{1, \ldots, l\}$ channels. Define the incidence matrix $A=\left[a_{i j}\right]$ where $a_{i j}=1$ if resource $i, i \in\{1, \ldots, m\}$, is used by product $j, a_{i j}=0$ otherwise. We denoted that $A_{j}$ is the set of legs used by product $j$ and $A^{i}$ is the set of products that use resource $i$. Therefore, the notation $i \in A_{j}$ indicates that leg $i$ is used by product $j$, and $j \in A^{i}$ indicates that product $j$ uses resource $i$. The initial seats of the resource $s$ is $s \in\left\{s_{1}, \ldots, s_{m}\right\}$. The state of the network is described by the remaining seats vector $x \in\left\{x_{1}, \ldots, x_{m}\right\}$. There are $t=\{0, \ldots T\}$ time periods in a finite selling horizon. Each time period is small enough such that maximum one customer will arrive in one period. Time starts from $t=0$ and ends at $t=T$. In each period, the customer arrives at $h$ channel and require $j$ product with probability $\lambda_{j h}$. It holds that $\lambda_{0}+\sum_{j=1}^{n} \sum_{h=1}^{l} \lambda_{j h}=1$, where $\lambda_{0}$ is the probability that no customer arrives at that time period. When one seat is sold by channel $\mathrm{h}$, the airline pays $C_{h} P_{j}$ commission fee to channel h. At the 
beginning of each period, when one demand comes in one channel requiring one product, the airline company first decides whether to accept the channel request or not. The decision is denoted by a binary variable $w_{h t}$. After that, the airline company makes the second decision about product request. The decision is denoted by another binary variable $u_{j t}$. If $u_{j t}=1$, the airline company accepts this product and 0 otherwise. After the customer request has been accepted, the airline company obtains the revenue $\left(1-C_{h}\right) P_{j}$. The decision depends on the remaining seats vector $x$, each time period $t$, the channel revenues rate $C_{h}$ and the revenue of products $P_{j}$. The objective is to maximize the total expected revenues in a selling horizon from the airline company's perspective. Now we formulated the decision problem as a dynamic programming: Parameters:

- $M$ : the set of resources in a network, indexed by i.

- $N$ : the set of products in a network, indexed by $\mathrm{j}$.

- $L$ : the set of channels in a network, indexed by $\mathrm{h}$.

- $A=\left[a_{i j}\right]$ : where $a_{i j}=1$ if resource $i$ is used by product $\mathrm{j}, a_{i j}=0$ otherwise.

- $x=\left(x_{1}, \ldots, x_{m}\right)$ : state vector of resource capacities, if product $j$ is sold, state change $x-A_{j}$.

- $t$ : represent each time period.

- $T$ : finite horizon periods.

- $s$ : initial seats od the resource, $s \in\left\{s_{1}, \ldots, s_{m}\right\}$

- $P(t)=\left(P_{1}(t), \ldots, P_{n}(t)\right)$ : revenue of the product, If $P_{j}(t)=P_{j}>0$, this indicates a request for product $j$ occurred and its associated price is $P_{j}$; If $P_{j}(t)=0$, this indicates there is no request for product $j$.

- $C=\left(C_{1}, \ldots, C_{h}\right)$ : Commission rate in channel $h$.

- $\lambda_{j h}(t)$ : the probability of customers arrival in products $j$ and channelh in time periods $t$.

- $w_{h t}$ : the channel decision binary indicator such that $w_{h t}=1$ if the airline company accepts channel $h$ request in time period $t$, and 0 otherwise.

- $u_{j t}$ : the product decision binary indicator such that $u_{j t}=1$ if the airline company accepts product $j$ request in time period $t$, and 0 otherwise.

- $V_{t}(x)$ : the maximum expected revenue of rest capacity $x$ in periods $t$.

Based on the parameters, the Bellman equation is then written as:

$$
\begin{array}{r}
V_{t}(x)=E\left[\operatorname { m a x } _ { u _ { j t } \in ( 0 , 1 ) , w _ { h t } \in ( 0 , 1 ) } \left\{P_{j} u_{j t}\left(1-C_{h}\right) W_{h t}\right.\right. \\
\left.\left.+V_{t+1}\left(x-A u_{j t} w_{h t}\right)\right\}\right] \\
\text { with boundary conditions } \\
V_{T}(x)=0 \quad \forall 0 \leq x \leq s \\
V_{t}(0)=0 \quad \forall 0 \leq t \leq T
\end{array}
$$


The boundary condition (5.13) represents that when the last period $\mathrm{T}$ comes, the airline will lost revenues of remaining seats. The boundary condition (5.14) illustrates that when the initial seats of flight has been sold out before time ends, the airline will not make any revenues. According to the definitions of $\lambda_{j h}$, the Bellman equation also can be rewritten as:

$$
\begin{array}{r}
V_{t}(x)=V_{t+1}(x)+\sum_{j \in N} \sum_{h \in L} \lambda_{j h}(t)\left[\left(\left(1-C_{h}\right) P_{j}\right.\right. \\
\left.\left.+V_{t+1}\left(x-A_{j}\right)-V_{t+1}(x)\right)^{+}\right]
\end{array}
$$

\subsubsection{Approximation method}

For any large scale network solving the DP model in exactly is practically hopeless because of the curse of dimensionality. For example, an airline network has 30 flight legs and each flight has 150 seats. The state of airline network will be $150^{3} 0$. Therefore, the problem has to rely on the approximation methods. One general approach is to use a deterministic approximation, in which stochastic demand are replaced by their mean demand. For example, the deterministic linear programming model(DLP) is simplified from the traditional dynamic network modelWilliamson(1992). Additionally the choice-bases linear programming model also was a deterministic approximation approach for choice-based DP formulation Liu and Van Ryzin(2008). Therefore, we also propose a deterministic Linear programming model to solve our channel network DP model and we call it NCDLP. In our NCDLP model, we let $D$ be the random vector of cumulative future demand at time t, and $E(D)$ its mean vector. In particular, $D_{j}$ represents the aggregate demand over the remaining $t$ time periods and $D_{h}$ represents the aggregate demand over the remaining $t$ time periods. We denote $y_{j}$ as the booking limit of capacity for product $j$. Additionally, we denote $z_{h j}$ as the booking limit of capacity for channel $h$. When a flight ticket is sold from channel $h$, the airline company will pay commission fee $C_{h} P_{j}$.

Additional Parameters:

- $y_{j}$ : the booking limit of product $j$.

- $z_{h j}$ : the booking limit of channel $h$ for product $j$.

- $E\left(D_{j}\right)$ : the aggregate mean demand of product $j$.

- $E\left(D_{h}\right)$ : the aggregate mean demand of channel $h$.

For any given capacity $x$ at time $t$, the deterministic linear programming model can be formulated as: 


$$
\begin{array}{r}
V_{L P}(x)=\max \sum_{j \in N} P_{j}\left(y_{j}-\sum_{h \in L} C_{h} z_{h j}\right) \\
\text { subject to: } \\
\sum_{j \in N} A_{i j} y_{j} \leq x_{i} \quad \forall i \in M \\
\sum_{j \in N} \sum_{h \in L} A_{i j} z_{h j} \leq x_{i} \quad \forall i \in M \\
y_{j} \leq E\left(D_{j}\right) \quad \forall j \in N \\
\sum_{j \in N} z_{h j} \leq E\left(D_{h}\right) \quad \forall h \in L \\
y_{j}=\sum_{h \in L} z_{h j} \quad \forall j \in N \\
y_{j} \geq 0 \quad \forall j \in N \\
z_{h j} \geq 0 \quad \forall j \in N, \forall h \in L
\end{array}
$$

In the DLP, the objective function is to maximize the total revenue minus channel commission fee. The constraint (5.17) states that the booking limit allocated to product booking requests must not violate the capacity limit on each resource $i$. The constraint (5.18) states that the booking limit for channel requests also must not violate the capacity limit on each resource $i$. The constraint (5.19) states that the booking limit of each product can not be over the demand of each product. The constraint (5.20) indicates that each channel's booking limit also can not be more than each channel's demand. The constraint (5.21) represents that each booking limit of product must equal to the sum of channel's booking limit on each product. The constraint (5.22) and (5.23) demonstrate that all booking limit are nonnegative number.

\subsection{Asymptotic optimality of the NCDLP}

As the Cooper(2002) shows that the he traditional DLP has the asymptotic property that its solution is asymptotically optimal for the network stochastic DP problem. Here, we will show the same property holds for NCDLP.

To do so, we restate the stochastic problem basing on the DP formulation 5.12. We define $y^{*}$ as the optimal solution and $y_{j}^{*}$ as the allocation capacity of the each fare class $j$. Define $V^{*}$ as the optimal expected revenue over the entire 
time horizon when applying the optimal solution $y^{*}$. With these definitions, the stochastic control problem can be written as:

$$
V^{*}=\max E\left[\sum_{j \in N} \sum_{h \in L} P_{j}\left(1-C_{h}\right) \min \left\{y_{j}^{*}, D_{j}\right\}\right]
$$

We first show that the objective value of the network channel determined linear problem 5.16 is the upper bound of the optimal expected revenue of the stochastic control problem 5.24.

Proposition $1 V^{*} \leq V_{L P}$

Proof 1 For any $j \in 1, \ldots, n$, we will have a cumulative vector of capacity allocation $y$ that satisfies:

$$
\sum_{j \in N} A_{i j} y_{j} \leq x_{i}
$$

And

$$
y_{j} \leq D_{j}
$$

According to the Jensen's inequality,

$$
V^{*}=\max E\left[\sum_{j \in N} \sum_{h \in L} P_{j}\left(1-C_{h}\right) \min \left\{y_{j}^{*}, D_{j}\right\} \leq \max \sum_{j \in N} \sum_{h \in L} P_{j}\left(1-C_{h}\right) E\left[\min \left\{y_{j}^{*}, D_{j}\right\}\right]\right.
$$

Additionally, we can know that $\max \sum_{j \in N} \sum_{h \in L} P_{j}\left(1-C_{h}\right) E\left[\min \left\{y_{j}^{*}, D_{j}\right\}\right]$ is a feasible solution of $V_{L P}$. So we can get that:

$$
V^{*} \leq V_{L P}
$$

Therefore, we can prove that NCDLP is the upper bound of the stochastic DP problem.

Next, we will prove that the upper bound NCDLP is asymptotically tight to the stochastic problem as both capacity and demand scaled up proportionately.

We consider a sequence of these problems and indexed them as $\vartheta=1,2, \ldots$. Additionally, in the $\vartheta$-scaled problem, the capacity is $\vartheta x$ and the demand is $\vartheta E\left[D_{j}\right]$. For the $\vartheta$-scaled problem, when $\vartheta=1$, the problem can be regarded as the original problem 5.16 and 5.24. We also define $V_{\vartheta}^{L P}$ as the expected revenue of the $\boldsymbol{\vartheta}$-scaled NCDLP problem and $V_{\vartheta}^{*}$ as the optimal revenue of the $\boldsymbol{\vartheta}$-scaled stochastic problem. We will get the property: 
Proposition 2

$$
\lim _{\vartheta \rightarrow \infty} \frac{1}{\vartheta} V_{\vartheta}^{*}=\lim _{\vartheta \rightarrow \infty} \frac{1}{\vartheta} V_{\vartheta}^{L P}=V_{L P}
$$

Proof 2 Firstly, we can define a sequence of the linear programs analogous to the function 5.16 as following equation:

$$
V_{\vartheta}^{L P}=\max \sum_{j \in N} P_{j}\left(\vartheta y_{j}-\sum_{h \in L} \vartheta C_{h} z_{h j}\right)
$$

As the proposition 1 , the $V_{\vartheta}^{L P}$ is also the upper bound of $V_{\vartheta}^{*}$ in the $\vartheta_{\text {-scaled }}$ problem. Therefore, $\vartheta y_{j}^{*}$ is the optimal solution to the scaled NCDLP problem. And we can get the $\vartheta$-scaled stochastic problem as following:

$$
V_{\vartheta}^{*}=\max E\left[\sum_{j \in N} \sum_{h \in L} P_{j}\left(1-C_{h}\right) \min \left\{\vartheta y_{j}^{*}, D_{j}(\vartheta)\right\}\right]
$$

Next we divide both formulation by $\vartheta$ and let $\vartheta \rightarrow \infty$, we can get:

$$
\begin{aligned}
& \lim _{\vartheta \rightarrow \infty} \frac{1}{\vartheta} V_{\vartheta}^{*}=\lim _{\vartheta \rightarrow \infty} \frac{1}{\vartheta} \sum_{j \in N} \sum_{h \in L} P_{j}\left(1-C_{h}\right) \min \left\{\vartheta y_{j}^{*}, D_{j}(\vartheta)\right\} \\
& =\lim _{\vartheta \rightarrow \infty} \sum_{j \in N} \sum_{h \in L} P_{j}\left(1-C_{h}\right) \min \left\{y_{j}^{*}, \frac{1}{\vartheta} D_{j}(\vartheta)\right\}
\end{aligned}
$$

When $\vartheta \rightarrow \infty, \frac{1}{\vartheta} D_{j}(\vartheta)=E\left[D_{j}\right]$ So:

$$
\begin{aligned}
& =\sum_{j \in N} \sum_{h \in L} P_{j}\left(1-C_{h}\right) \min \left\{y_{j}^{*}, E\left[D_{j}\right]\right\} \\
& \left.=\sum_{j \in N} \sum_{h \in L} P_{j}\left(1-C_{h}\right) y_{j}^{*}\right\} \\
& =\sum_{j \in N} P_{j}\left(y_{j}^{*}-\sum_{h \in L} C_{h} z_{h j}^{*}\right) \\
& =V_{L P}
\end{aligned}
$$

Therefore, the proven results shows that, the optimal revenue of the $\boldsymbol{\vartheta}$-scaled problem converges in $\vartheta$ infinity to the upper bound $V_{L P}$. Thus, the revenue of the determined linear programming can converge to the optimal revenue. 


\subsection{Numerical examples}

In this section, we conducted numerical experiment using an airline network to verify the performance of our model. In figure 5.2, a small airline network has three cities and three flight legs.

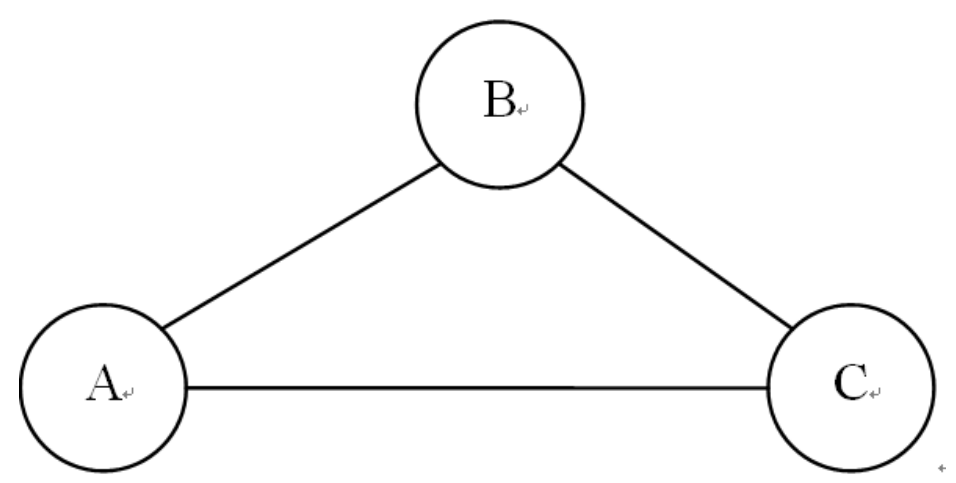

Figure 5.2: Network for experiment

Additionally each flight has its own capacities which are shown in table 5.1. Each flight consists of two fare classes, so the airline network has six products as in table 5.2. Mean demands of each product are assumed as 1.5 demand ratio which equals to 1.5 times of capacity.

Table 5.1: Resource Characteristics for Experiment

\begin{tabular}{c|cc}
\hline Resource & Leg & Capacity \\
\hline 1 & A-B & 30 \\
2 & B-C & 16 \\
3 & A-C & 15 \\
\hline
\end{tabular}

In the experiment, we illustrate the traditional determined linear programming model(DLP, see Williamson,1992) as the benchmark for the comparison purpose. More specifically, DLP model only can allocate the capacity in fare class. The products and resource characteristics are the same as the new channel DLP model. The channel part in the reality depends on the rule of first come first serve as we mentioned above. The products and resource characteristics are the same as the new channel DLP model. The data in Table 5.3 are shown the channel characteristics. In the example, we assume that the airlines have two channels: channel 1 which represents indirect channel such as travel agency whereas channel 2 represents direct channel such as airline websites. These two channels differ in terms of their demand and commission rate. To test the effect 
Table 5.2: Products Characteristics for Experiment

\begin{tabular}{c|cccc}
\hline Products & Origin-Destination & Class & Fare & Mean Demand \\
\hline 1 & A-C & H & 1200 & 9 \\
2 & A-B-C & H & 800 & 9 \\
3 & A-B & H & 600 & 12 \\
4 & A-C & L & 800 & 14 \\
5 & A-B-C & L & 500 & 15 \\
6 & A-B & L & 300 & 9 \\
\hline
\end{tabular}

of channel on airlines revenue, we run 5 instances with different commission rate(See Table 5.3).

Table 5.3: Channel Characteristics for Experiment

\begin{tabular}{c|cc}
\hline Channel & Commission Rate & Mean Demand \\
\hline 1 & $0.15,0.25,0.35,0.45,0.55$ & 54 \\
2 & 0 & 14 \\
\hline
\end{tabular}

As shown in figure 5.3, the new channel DLP model makes more revenues than the traditional DLP model in different commission rate situation. For example, when the commission rate is 0.15 , the new model has $3.99 \%$ revenue improvements. With the commission rate increasing, the new model can make more revenue improvements. According to this point, new model can keep the revenues more stable when the commission rate increase.

\subsection{Conclusion}

In this Chapter, we have studied the problem of capacity control with flight fare class and multi-channel distribution in the network environment. The network dynamic model of Talluri and Van Ryzin(1998) is a general and traditional capacity allocation model, which is widely used in airlines industry. Here, we have extended their model to solve the network model under the multi-channel environment. We proposed a new network dynamic model and introduced the channel factor into the new model. As we mentioned in the section 5.2.2, the dynamic programming model is hardly to be solved under the large scale network environment. Therefore, we have developed an approximation method to 


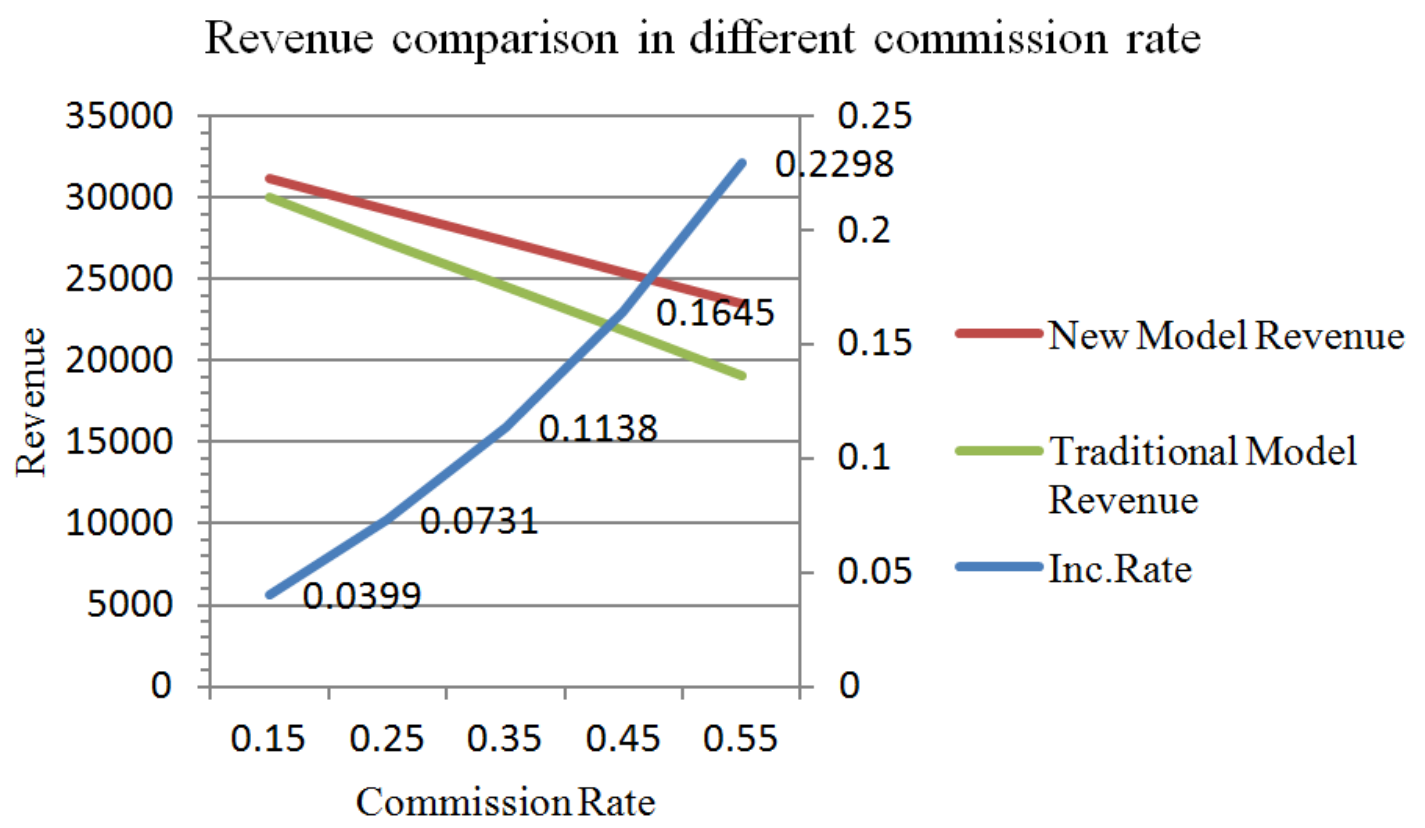

Figure 5.3: Revenue comparison in different commission rate

solve the problem instead of dynamic programming model. The approximation method is the determined linear programming model which is widely used to solve the network dynamic programming problem in revenue management. The numerical experiments illustrate that the determined linear program can be solved optimally. Additionally, the results proved that the network model which considered channel factor can make more improvements on revenues for airlines than the traditional network model. Some implications from single-leg model have also been showed in the channel network model. For example, the model which considered channel factor make the revenue system more stable when the commission fee changes than the traditional model. This implications also can be showed in the results of new network model. Therefore, the key contribution of this study is that we introduce the channel distribution into the traditional network revenue management. On the base of this we proposed an approximation method to make this problem be solved and make the model be used in practical. Thus, this study improves the development of revenue management. 
6 Conclusion and future study

6.1 Conclusion

With the rapid development of e-commerce, it has brought opportunities and challenges to every industry. The biggest challenge among these is the change of marketing channels. The airlines marketing channels are different compared with marketing channels of traditional products. Airlines compete tremendously in increasing customer volume and maintaining market share in a limited time period. Hence, airlines utilize many channels to reach customers in order to enhance the sales opportunities. However, with the development of OTA, the airlines have to pay high commission fee for each ticket they sold. Although the airlines realize that this is a serious problem for airlines and have adopted strategies to increase the sales proportion of direct channel, the strategies is not obviously useful. Under this current situation, airlines have to pay a large amount of commission fee for the indirect channel distribution every year. Hence, how to reduce this cost of channel distribution has become a focus issue from the perspective of some airline companies.

At the same time, the airline's 40-year revenue management strategy has also required radical changes to adapt to the new Internet environment. In the beginning, revenue management is used typically to determine how many seats should be reserved and offered for each class at different prices in one aircraft cabin. Since the application of this management technology, American airlines have acquired more profits in 1980s during which period almost all other airlines had a great deficit. Although revenue management often assumes that cost is not considered because of the high fixed cost and low variable cost, this assumption does not necessarily true concern the cost of channel distribution in nowadays market. Eventually, the channel cost will significantly affect the revenue of selling tickets, and therefore this is essential to investigate how the channel distribution affects the decision making of airlines' revenue management system.

On this basis, this thesis has done related research and reached the following conclusions:

Firstly, we have studied the problem of capacity control in the single-leg flight fare class and multi-channel distribution. We proposed a dynamic programming model for integrating the fare class capacity allocation and the channel capacity deployment. After that, we have explored the optimal policy for the integrated model to maximum the airlines revenues and proposed several properties corresponding to the optimal policy. Furthermore, we proposed a customer-shift 
behaviour model on the basis of the integrated model and discovered the optimal policy for that. The numerical experiments illustrate the different applications of the model and provide several implications for airline companies. Firstly, our system can keep the revenues more stable when channel commission rates increase. This implication decreases the lost revenue risk by contracting with the channel and holding the dominant position in airline market. Secondly, multiple indirect sales channels will not reduce airline company revenues, and, conversely, more indirect channels might bring more profits for the airline company. Therefore, the airlines should introduce more channels to improve their revenues. Thirdly, the demand forecast dominates a significant position in our system. The accurate prediction of demand improves the revenues for the airline company. After that, our system integrates the channel and fare class, and there is not an obvious conflict between the channel and fare class allocation. The airline company can realize channel control on the basis of the revenue system. However, we need to note the observation that the increased rate of the DCAM with multi-fare and without multi-fare brings a small difference. This might be due to the combined but complex effects of the fare class and the channel distribution. As discussed in Section 5.4, the assumption of customer arrival is one explanation for the result. A more thorough investigation is needed in a future study. Thus, our study solves a mathematical problem, and it has value for practical use. Finally, all applications we proposed have better performance in dealing with the problem of multi-fare and multi-channel capacity control than the classic independent system. The key contribution of this study is that it is the first attempt to integrate the capacity allocation decision and distribution channel decision in order to improve the revenue management. The modelling framework provides a basis for investigating relevant issues in future studies. The optimal policy together with the observations from numerical examples brings insights on the directions where revenues can be improved in practice. Thus, from both the theoretical and practical perspectives, this study contributes to the knowledge development in the field.

Secondly, we studied a multi-channel single-leg capacity allocation model that considers customer channel-shift behaviour. We propose a model that introduces customer transfer behaviour into multi-channel capacity allocation. After that, we have developed the optimal policy on the base of multi-channel capacity allocation optimal policy. The numerical experiments enumerate the impact of the model on the revenue management system under different circumstances and suggest various inspirations to the airlines. Firstly, Our system revenue will increase as customer transfer rates increase. This has a great effect on the revenue system considering multi-channel issues because the channel's transfer behaviour has no loss to the customer itself. When the customer discovers that a channel cannot purchase a ticket and transfer to other channels 
without any cost, there is a high probability that they will choose to purchase tickets through other channels. For airlines, it makes sense to collect transfer customer demands and make some research. Secondly, the model increases the acceptance rate of customers to reduce customer loss caused by the introduction of multi-channel factor. Retaining more customers means improving the competitiveness of airlines. Thirdly, The model that considers customer transfer behaviour has better stability than the model that does not consider customer transfer behaviour. When agency fees increase, airlines can better reduce cost increases. Fourthly, The customer's transfer behaviour has a great influence on the demand forecast. Airlines can reduce sales costs by changing sales time through accurate demand forecasting or increase airline revenue by replacing larger aircraft. Finally, our model not only has better results than the traditional revenue management model, but also has better results than the multi-channel model. Our model is also more realistic than other models.

Thirdly, we have studied the problem of capacity control with flight fare class and multi-channel distribution in the network environment. The network dynamic model of Talluri and van Ryzin(1999) is a general and traditional capacity allocation model, which is widely used in airlines industry. Here, we have extended their model to solve the network model under the multi-channel environment. We proposed a new network dynamic model and introduced the channel factor into the new model. As we mentioned in the section 4, the dynamic programming model is hardly to be solved under the large scale network environment. Therefore, we have developed an approximation method to solve the problem instead of dynamic programming model. The approximation method is the determined linear programming model which is widely used to solve the network dynamic programming problem in revenue management. The numerical experiments illustrate that the determined linear program can be solved optimally. Additionally, the results proved that the network model which considered channel factor can make more improvements on revenues for airlines than the traditional network model. Some implications from single-leg model have also been showed in the channel network model. For example, the model which considered channel factor make the revenue system more stable when the commission fee changes than the traditional model. This implications also can be showed in the results of new network model. Therefore, the key contribution of this study is that we introduce the channel distribution into the traditional network revenue management. On the base of this we proposed an approximation method to make this problem be solved and make the model be used in practical. Thus, this study improves the development of revenue management. 
There are many possible directions for future study in the channel and capacity allocation. Understanding the competitive behaviours between different channels under the channel and fare system is a significant topic for future investigation. In our model, we only considered capacity allocation and determined price strategy. As a further extension, price strategy can be included in the revenue system. Except for the airline industry, other industries (such as the hotel industry, high speed rail industry, car rental industry and some retail markets) are also of interest for the application of the knowledge developed in this study. However, we need to note that our experiments only examines in a small network. In the future study, the model need to be testified in a large and more complicated network. Apart from this, the future study also can consider the competition effect between different channels. 


\section{Acknowledgement}

After five years of doctoral career, there are too many teachers, classmates, friends and relatives who need to thank.

At first, I would like to thank my two supervisors, Prof.Huo Jiazhen and Prof.Hans-Christian Pfohl. Without their support and encouragement, I would not be able to stick to get the Ph.D. After that, I would like to thank Prof.Tang Ou. When I was in the hardest and most helpless periods, he helped me and guided me a lot. I would also like to thank Prof. Liang zhe, Prof. Li Xiangyong, Prof. Duan Yon- grui. They gave me a lot of support in my research period. I am also grateful to my fellow doctoral students,Pascal Wolff,Johannes Kern, Qin Ying and Hou Yanting. They always could give me some good feedback and also we have a good corporation and friendship.

I would like to thank all of my good friends in Tongji University, Wang Daqin, Zhang Lihua, Chen Yiran, Zhang Yanxia. I am so grateful to the friends in Darmstadt, Lv Suwei, Xue Xiufeng, Zhu Feng. They accompanied me through the darkest hours in Germany. The last but the most important, I would like to thank my parents. Without their support, I will never never never get the $\mathrm{Ph}$.D. I am also very grateful to my little family,my wife and my son. They are my spiritual pillar. 
Bibliography

[1] Niels AH Agatz, Moritz Fleischmann, and Jo AEE Van Nunen. Efulfillment and multi-channel distribution-a review. European journal of operational research, 187(2):339-356, 2008.

[2] Fariba Alamdari and Keith Mason. The future of airline distribution. Journal of Air Transport Management, 12(3):122-134, 2006.

[3] Aydın Alptekinoğlu and Christopher S Tang. A model for analyzing multichannel distribution systems. European Journal of Operational Research, 163(3):802-824, 2005.

[4] Sven-Eric Andersson. Passenger choice analysis for seat capacity control: a pilot project in scandinavian airlines1. International Transactions in Operational Research, 5(6):471-486, 1998.

[5] A Muzaffer Arslan, JBG Frenk, and Semih O Sezer. On the single-leg airline revenue management problem in continuous time. Mathematical Methods of Operations Research, 81(1):27-52, 2015.

[6] Nurşen Aydın, Ş İlker Birbil, JBG Frenk, and Nilay Noyan. Single-leg airline revenue management with overbooking. Transportation Science, 47(4):560-583, 2012.

[7] Nurşen Aydın, Ş İlker Birbil, and Hüseyin Topaloğlu. Delayed purchase options in single-leg revenue management. Transportation Science, 51(4): 1031-1045, 2016.

[8] Mehmet Barut and V Sridharan. Design and evaluation of a dynamic capacity apportionment procedure. European Journal of Operational Research, 155(1):112-133, 2004.

[9] Mehmet Barut and V Sridharan. Revenue management in order-driven production systems. Decision sciences, 36(2):287-316, 2005.

[10] Peter Belobaba. Air travel demand and airline seat inventory management. PhD thesis, Massachusetts Institute of Technology, 1987.

[11] Peter P Belobaba. Survey paper?airline yield management an overview of seat inventory control. Transportation science, 21(2):63-73, 1987.

[12] Peter P Belobaba. Or practice?application of a probabilistic decision model to airline seat inventory control. Operations Research, 37(2):183197, 1989. 
[13] PP Belobaba. Revenue and competitive impacts of od control: Summary of pods results. In First Annual INFORMS Revenue Management Section Meeting, New York, NY, 2001.

[14] PP Belobaba and S Lee. Pods update: Large network od control results. In Proc. 2000 AGIFORS Reservations and Yield Management Study Group Symposium. New York, NY, 2000.

[15] Dimitris Bertsimas and Sanne De Boer. Simulation-based booking limits for airline revenue management. Operations Research, 53(1):90-106, 2005.

[16] Dimitris Bertsimas and Ioana Popescu. Revenue management in a dynamic network environment. Transportation science, 37(3):257-277, 2003.

[17] Dimitris Bertsimas and Romy Shioda. Restaurant revenue management. Operations research, 51(3):472-486, 2003.

[18] AV Bhatia and SC Parekh. Optimal allocation of seats by fare. Presentation by Trans World Airlines to AGIFORS Reservations Study Group, 1973.

[19] Enrique Bigné, Blanca Hernández, Carla Ruiz, and Luisa Andreu. How motivation, opportunity and ability can drive online airline ticket purchases. Journal of Air Transport Management, 16(6):346-349, 2010.

[20] Juan José Miranda Bront, Isabel Méndez-Díaz, and Gustavo Vulcano. A column generation algorithm for choice-based network revenue management. Operations Research, 57(3):769-784, 2009.

[21] Shelby Brumelle and Darius Walczak. Dynamic allocation of airline seat inventory with batch arrivals. NASA, (19980018508), 1997.

[22] Shelby Brumelle and Darius Walczak. Dynamic airline revenue management with multiple semi-markov demand. Operations Research, 51(1): 137-148, 2003.

[23] Shelby L Brumelle and Jeffrey I McGill. Airline seat allocation with multiple nested fare classes. Operations research, 41(1):127-137, 1993.

[24] Shelby L Brumelle, Jeffrey I McGill, Tae Hoon Oum, K Sawaki, and MW Tretheway. Allocation of airline seats between stochastically dependent demands. Transportation Science, 24(3):183-192, 1990.

[25] Dimitrios Buhalis and Peter O'Connor. Information communication technology revolutionizing tourism. Tourism recreation research, 30(3):7-16, 2005 . 
[26] Burak Büke, Utku Yildirim, and Harun Ahmet Kuyumcu. New stochastic linear programming approximations for network capacity control problem with buy-ups. Journal of Revenue and Pricing Management, 7(1):61-84, 2008.

[27] CAST. China Civil Aviation Statistics Compilation. China Civil Aviation Press, Beijing, 2017.

[28] José I Castillo-Manzano and Lourdes López-Valpuesta. The decline of the traditional travel agent model. Transportation Research Part E: Logistics and Transportation Review, 46(5):639-649, 2010.

[29] Victoria CP Chen, Dirk Günther, and Ellis L Johnson. A markov decision problem based approach to the airline ym problem. In YM Problem, Working Paper, The Logistics Institute, Georgia Institute of Technology. Citeseer, 1998.

[30] Yung-Hsiang Cheng and Ting-Yu Huang. High speed rail passenger segmentation and ticketing channel preference. Transportation Research Part A: Policy and Practice, 66:127-143, 2014.

[31] Wei-yu Kevin Chiang, Dilip Chhajed, and James D Hess. Direct marketing, indirect profits: A strategic analysis of dual-channel supply-chain design. Management science, 49(1):1-20, 2003.

[32] Wen-Chyuan Chiang, Jason CH Chen, and Xiaojing Xu. An overview of research on revenue management: current issues and future research. International journal of revenue management, 1(1):97-128, 2006.

[33] Sunmee Choi and Sheryl E Kimes. Electronic distribution channels? effect on hotel revenue management. Cornell Hotel and Restaurant Administration Quarterly, 43(3):23-31, 2002.

[34] A Ciancimino, G Inzerillo, Stefano Lucidi, and Laura Palagi. A mathematical programming approach for the solution of the railway yield management problem. Transportation science, 33(2):168-181, 1999.

[35] William L Cooper. Pathwise properties and performance bounds for a perishable inventory system. Operations Research, 49(3):455-466, 2001.

[36] William L Cooper. Asymptotic behavior of an allocation policy for revenue management. Operations Research, 50(4):720-727, 2002.

[37] William L Cooper and Le Li. On the use of buy up as a model of customer choice in revenue management. Production and Operations Management, 21(5):833-850, 2012. 
[38] William L Cooper, Tito Homem-de Mello, and Anton J Kleywegt. Models of the spiral-down effect in revenue management. Operations Research, 54(5):968-987, 2006.

[39] Renwick E Curry. Optimal airline seat allocation with fare classes nested by origins and destinations. transportation science, 24(3):193-204, 1990.

[40] Sanne De Boer, Richard Freling, and Nanda Piersma. Stochastic programming for multiple-leg network revenue management. Technical report, 1999.

[41] Moshe Dror, Pierre Trudeau, and Shaul P Ladany. Network models for seat allocation on flights. Transportation Research Part B: Methodological, 22(4):239-250, 1988.

[42] E. D'Sylva. Od seat assignment to maximize expected revenue. Technical report, Boeing Commercial Airplane Company, Seattle, WA, 1982., 1982.

[43] Sharbel El-Haber and Muhammad El-Taha. Dynamic two-leg airline seat inventory control with overbooking, cancellations and no-shows. Journal of Revenue and Pricing Management, 3(2):143-170, 2004.

[44] Jacob B Feldman and Huseyin Topaloglu. Revenue management under the markov chain choice model. Operations Research, 65(5):1322-1342, 2017.

[45] Youyi Feng and Baichun Xiao. A dynamic airline seat inventory control model and its optimal policy. Operations Research, 49(6):938-949, 2001.

[46] Paraskevi Fountoulaki, M Claudia Leue, and Timothy Jung. Distribution channels for travel and tourism: The case of crete. pages 667-680, 2015.

[47] Guillermo Gallego, Lin Li, and Richard Ratliff. Choice-based emsr methods for single-leg revenue management with demand dependencies. Journal of Revenue and Pricing Management, 8(2-3):207-240, 2009.

[48] Guillermo Gallego, Richard Ratliff, and Sergey Shebalov. A general attraction model and sales-based linear program for network revenue management under customer choice. Operations Research, 63(1):212-232, 2014.

[49] Fred Glover, Randy Glover, Joe Lorenzo, and Claude McMillan. The passenger-mix problem in the scheduled airlines. Interfaces, 12(3):73-80, 1982.

[50] Dong Ling Han, Loon Ching Tang, and Huei Chuen Huang. A markov model for single-leg air cargo revenue management under a bid-price policy. European Journal of Operational Research, 200(3):800-811, 2010. 
[51] Henry H Harteveld. The Future of Airline Distribution: A Look Ahead to 2017: a Special Report Commussioned by IATA. Atmosphere Research Group LLC, 2012.

[52] Henry H Harteveld. The Future of Airline Distribution: 2016-2021. Atmosphere Research Group LLC, 2016.

[53] Daniel Hopman, Ger Koole, and Rob van der Mei. Single-leg revenue management with downsell and delayed decision making. Journal of Revenue and Pricing Management, 16(6):594-606, 2017.

[54] Scott Hornick. Fare play. Airline Business, 20:72-75, 2004.

[55] David Jarach. The digitalisation of market relationships in the airline business: the impact and prospects of e-business. Journal of Air Transport Management, 8(2):115-120, 2002.

[56] Houyuan Jiang and Giovanna Miglionico. Airline network revenue management with buy-up. Optimization, 63(6):849-865, 2014.

[57] Samuel Nathan Kirshner and Mikhail Nediak. Scalable dynamic bid prices for network revenue management in continuous time. Production and Operations Management, 24(10):1621-1635, 2015.

[58] Robert Klein. Network capacity control using self-adjusting bid-prices. or spectr. Operations Research-Spektrum, 29(1):39-60, 2006.

[59] Anton J Kleywegt and Jason D Papastavrou. The dynamic and stochastic knapsack problem with random sized items. Operations Research, 49(1): 26-41, 2001.

[60] Bonwoo Koo, Benny Mantin, and Peter O'Connor. Online distribution of airline tickets: Should airlines adopt a single or a multi-channel approach? Tourism Management, 32(1):69-74, 2011.

[61] Sumit Kunnumkal and Huseyin Topaloglu. A new dynamic programming decomposition method for the network revenue management problem with customer choice behavior. Production and Operations Management, 19 (5):575-590, 2010.

[62] A Kuyumcu and A Garcia-Diaz. A polyhedral graph theory approach to revenue management in the airline industry. Computers \& industrial engineering, 38(3):375-395, 2000.

[63] Conrad J Lautenbacher and Shaler Stidham Jr. The underlying markov decision process in the single-leg airline yield-management problem. Transportation Science, 33(2):136-146, 1999. 
[64] Tak C Lee and Marvin Hersh. A model for dynamic airline seat inventory control with multiple seat bookings. Transportation science, 27(3):252265, 1993.

[65] Yigao Liang. Solution to the continuous time dynamic yield management model. Transportation Science, 33(1):117-123, 1999.

[66] K LITTLEWOOD. Forecasting and control of passengers. In 12th AGIFORS Symposium Proceedings, volume 95, page 128, 1972.

[67] Qian Liu and Garrett Van Ryzin. On the choice-based linear programming model for network revenue management. Manufacturing \& Service Operations Management, 10(2):288-310, 2008.

[68] Michael Mayer. Seat allocation, or a simple model of seat allocation via sophisticated ones. In AGIFORS Symposium Proceedings, volume 16, pages 339-362, 1976.

[69] Jeffrey I McGill. Optimization and estimation problems in airline yield management. PhD thesis, University of British Columbia, 1989.

[70] Jeffrey I McGill and Garrett J Van Ryzin. Revenue management: Research overview and prospects. Transportation science, 33(2):233-256, 1999.

[71] Joern Meissner and Arne Strauss. Network revenue management with inventory-sensitive bid prices and customer choice. European Journal of Operational Research, 216(2):459-468, 2012.

[72] Andris Möller, Werner Römisch, and Klaus Weber. A new approach to o\&d revenue management based on scenario trees. Journal of Revenue and Pricing Management, 3(3):265-276, 2004.

[73] Kevin Pak and Nanda Piersma. Overview of or techniques for airline revenue management. Statistica Neerlandica, 56(4):479-495, 2002.

[74] Phillip E Pfeifer. The airline discount fare allocation problem. Decision Sciences, 20(1):149-157, 1989.

[75] Robert L Phillips. A marginal-value approach to airline origin \& destination revenue management. In System Modelling and Optimization, pages 905-917. Springer, 1994.

[76] Robert L Phillips, Dean W Boyd, and Thomas A Grossman Jr. An algorithm for calculating consistent itinerary flows. Transportation Science, 25(3):225-239, 1991. 
[77] Robert Lewis Phillips. Pricing and revenue optimization. Stanford University Press, 2005.

[78] Arvind Rangaswamy and Gerrit H Van Bruggen. Opportunities and challenges in multichannel marketing: An introduction to the special issue. Journal of Interactive Marketing, 19(2):5-11, 2005.

[79] B Venkateshwara Rao and Barry C Smith. Decision support in online travel retailing. Journal of Revenue and Pricing Management, 5(1):72-80, 2006.

[80] Martin I Reiman and Qiong Wang. An asymptotically optimal policy for a quantity-based network revenue management problem. Mathematics of Operations Research, 33(2):257-282, 2008.

[81] Helmut Richter. The differential revenue method to determine optimal seat allotments by fare type. In AGIFORS PROCEEDINGS, 1982.

[82] Lawrence W Robinson. Optimal and approximate control policies for airline booking with sequential nonmonotonic fare classes. Operations Research, 43(2):252-263, 1995.

[83] Carla Ruiz-Mafe, Silvia Sanz-Blas, and Joaquín Aldás-Manzano. Drivers and barriers to online airline ticket purchasing. Journal of Air Transport Management, 15(6):294-298, 2009.

[84] A Shaykevich. Airline yield management: Dynamic programming approach. Master's thesis, Department of Operations Research, University of North Carolina, Chapel Hill, NC, 1994.

[85] Zheng-Yi Shon, Fang-Yuan Chen, and Yu-Hern Chang. Airline ecommerce: the revolution in ticketing channels. Journal of Air Transport Management, 9(5):325-331, 2003.

[86] Robert A Shumsky and Fuqiang Zhang. Dynamic capacity management with substitution. Operations research, 57(3):671-684, 2009.

[87] Dirk Sierag and Rob van der Mei. Single-leg choice-based revenue management: a robust optimisation approach. Journal of Revenue and Pricing Management, 15(6):454-467, 2016.

[88] David Simchi-Levi, S David Wu, and Zuo-Jun Max Shen. Handbook of quantitative supply chain analysis: modeling in the e-business era, volume 74. Springer Science \& Business Media, 2004.

[89] RW Simpson. Using network flow techniques for origin-destination seat inventory control. Memorandum M89-1. Flight Transportation Laboratory, Massachusetts Institute of Technology, Cambridge, 1989. 
[90] Barry C Smith, Dirk P Günther, B Venkateshwara Rao, and Richard M Ratlife. E-commerce and operations research in airline planning, marketing, and distribution. Interfaces, 31(2):37-55, 2001.

[91] BC Smith and CW Penn. Analysis of alternate origin-destination control strategies. In AGIFORS PROCEEDINGS, 1988.

[92] Arne K Strauss, Robert Klein, and Claudius Steinhardt. A review of choice-based revenue management: Theory and methods. European Journal of Operational Research, 2018.

[93] Janakiram Subramanian, Shaler Stidham Jr, and Conrad J Lautenbacher. Airline yield management with overbooking, cancellations, and no-shows. Transportation science, 33(2):147-167, 1999.

[94] Kalyan Talluri. New formulations for choice network revenue management. INFORMS Journal on Computing, 26(2):401-413, 2014.

[95] Kalyan Talluri and Garrett Van Ryzin. An analysis of bid-price controls for network revenue management. Management Science, 44(11-part-1): 1577-1593, 1998.

[96] Kalyan Talluri and Garrett Van Ryzin. A randomized linear programming method for computing network bid prices. Transportation science, 33(2): 207-216, 1999.

[97] Kalyan Talluri and Garrett Van Ryzin. Revenue management under a general discrete choice model of consumer behavior. Management Science, 50(1):15-33, 2004.

[98] Kalyan T Talluri and Garrett J Van Ryzin. The theory and practice of revenue management, volume 68. Springer Science \& Business Media, 2006.

[99] Bernard Titze and Raimund Griesshaber. Realistic passenger booking behaviour and the simple low-fare high-fare seat allotment model. In AGIFORS PROCEEDINGS-, 1983.

[100] Rex S Toh and Peter Raven. Perishable asset revenue management: Integrated internet marketing strategies for the airlines. Transportation Journal, pages 30-43, 2003.

[101] Huseyin Topaloglu. Using lagrangian relaxation to compute capacitydependent bid prices in network revenue management. Operations Research, 57(3):637-649, 2009. 
[102] Tony Tyler. IATA Annual Review 2016. International Air Transport Association, 2016.

[103] Garrett Van Ryzin and Jeff McGill. Revenue management without forecasting or optimization: An adaptive algorithm for determining airline seat protection levels. Management Science, 46(6):760-775, 2000.

[104] Richard Van Slyke and Yi Young. Finite horizon stochastic knapsacks with applications to yield management. Operations Research, 48(1):155-172, 2000 .

[105] Thomas WM Vossen and Dan Zhang. Reductions of approximate linear programs for network revenue management. Operations Research, 63(6): 1352-1371, 2015.

[106] David W Wallace, Joan L Giese, and Jean L Johnson. Customer retailer loyalty in the context of multiple channel strategies. Journal of retailing, 80(4):249-263, 2004.

[107] Ken Wang. Optimum seat allocation for multi-leg flights with multiple fare types. In AGIFORS PROCEEDINGS-, 1983.

[108] Lawrence R Weatherford, Samuel E Bodily, and Phillip E Pfeifer. Modeling the customer arrival process and comparing decision rules in perishable asset revenue management situations. Transportation Science, 27(3):239251, 1993.

[109] Xia Yusen Wei Yihua, Hu Qiying. A continuous time revenue management problem with multi-channels. Chinese journal of management science, 16: 37-41, 2008.

[110] Elizabeth Louise Williamson. Comparison of optimization techniques for origin-destination seat inventory control. Technical report, [Cambridge, Mass.: Massachusetts Institute of Technology], Flight Transportation Laboratory,[1988], 1988.

[111] Elizabeth Louise Williamson. Airline network seat inventory control: Methodologies and revenue impacts. PhD thesis, Massachusetts Institute of Technology, 1992.

[112] RD Wollmer. A hub-spoke seat management model. Unpublished Internal Report, Mc Donnell Douglas Corporation, Long Beach, CA, 1986.

[113] Richard D Wollmer. An airline seat management model for a single leg route when lower fare classes book first. Operations Research, 40(1):26-37, 1992. 
[114] J Wong. Airline network seat allocation. 1990.

[115] Jinn-Tsai Wong, Frank S Koppelman, and Mark S Daskin. Flexible assignment approach to itinerary seat allocation. Transportation Research Part B: Methodological, 27(1):33-48, 1993.

[116] R Wysong. A simplified method for including network effects in capacity control. In AGIFORS Symposium Proc, volume 28, 1988.

[117] Wenming Xie, Zhibin Jiang, Yingxue Zhao, and Junjie Hong. Capacity planning and allocation with multi-channel distribution. International Journal of Production Economics, 147:108-116, 2014.

[118] Dong-Qing Yao and John J Liu. Channel redistribution with direct selling. European Journal of Operational Research, 144(3):646-658, 2003.

[119] Moon Gil Yoon, Duk Young Yoon, and Tae Won Yang. Impact of ebusiness on air travel markets: Distribution of airline tickets in korea. Journal of Air Transport Management, 12(5):253-260, 2006.

[120] Peng-Sheng You. Airline seat management with rejection-for-possibleupgrade decision. Transportation Research Part B: Methodological, 35 (5):507-524, 2001.

[121] PS You. Dynamic pricing of inventory with cancellation demand. Journal of the Operational Research Society, 54(10):1093-1101, 2003.

[122] Y Young and R Van Slyke. Stochastic knapsack models of yield management. 1994.

[123] Dan Zhang and Daniel Adelman. An approximate dynamic programming approach to network revenue management with customer choice. Transportation Science, 43(3):381-394, 2009.

[124] W Zhao. Dynamic and static yield management systems. PhD thesis, Ph. D thesis, Wharton School, University of Pennsylvania, 1999.

[125] Wen Zhao and Yu-Sheng Zheng. A dynamic model for airline seat allocation with passenger diversion and no-shows. Transportation Science, 35 (1):80-98, 2001. 